\title{
Characteristics of the Innovation, Organization, Environment and Individual that Facilitate the Utilization of Five Systematic Overviews Among Public Health Decision-Makers in Ontario
}

Sept 24, 1999

Maureen Dobbins, RN, BScN

A thesis submitted in conformity with the requirements for the degree of Doctor of Philosophy Graduate Department of Health Administration University of Toronto

This research was made possible by Health Canada through a National Health Research and Development Program (NHRDP) Research Training Award, The Central East Health Information Partnership (CEHIP), and the Hamilton-Wentworth Regional Department of Public Health Services

(C) Copyright by Maureen Jennifer Dobbins 1999 
National Library

of Canada

Acquisitions and Bibliographic Services

395 Wellington Street Ottawa ON K1A ON4 Canada
Bibliothèque nationale du Canada

Acquisitions et services bibliographiques

395. rue Wellington Onawa ON KIA ONA

Canada
The author has granted a nonexclusive licence allowing the National Library of Canada to reproduce, loan, distribute or sell copies of this thesis in microform, paper or electronic formats.

The author retains ownership of the copyright in this thesis. Neither the thesis nor substantial extracts from it may be printed or otherwise reproduced without the author's permission.
L'auteur a accordé une licence non exclusive permettant à la Bibliothèque nationale du Canada de reproduire, prêter, distribuer ou vendre des copies de cette thèse sous la forme de microfiche/film, de reproduction sur papier ou sur format électronique.

L'auteur conserve la propriété du droit d'auteur qui protège cette thèse. $\mathrm{Ni}$ la thèse ni des extraits substantiels de celle-ci ne doivent être imprimés ou autrement reproduits sans son autorisation. 
Characteristics of the Innovation, Organization, Environment and Individual that Facilitate the Utilization of Five Systematic Overviews Among Public Heaith

Decision-Makers in Ontario

Doctor of Philosophy 1999

Maureen Dobbins

Graduate Department of Health Administration

University of Toronto

\begin{abstract}
Obiectives: To determine which individual, organizational, environmental, and characteristics of the innovation are most strongly associated with systematic overviews influencing policy decisions related to program planning, justification, evaluation, policy development and staff development among individual decision-makers in Ontario.

Methods: This cross-sectional study included a telephone survey of senior public health decision-makers in all 41 public health units in Ontario, who had been disseminated 5 systematic overviews two years earlier. A short, self-administered organizational demographics questionnaire was also distributed to the administrative assistant of each Medical Officer of Health for each health unit. Multiple logistic and linear regression analyses were conducted to identify significant predictors of the overall use of the systematic overviews, and the influence of these overviews on policy decisions.
\end{abstract}

Results: The response rate was high at $87 \%$. Perception of characteristics of the innovation such as the relevance of the systematic overviews to decisions being made and whether they were easy to use proved to be the most significant predictors of systematic overview use and their influence on public health decisions. The value the organization placed on research evidence, an organizational characteristic, was the third most important variable to predict the research 
utilization outcomes examined in this study. The explanatory power of the multiple linear regression models were high with $15 \%$ to $40 \%$ of the variation in the influence of the overviews on public health decisions being explained by the models. The individual and environmental characteristics exened the least impact on the research utilization outcomes.

Conclusions: The results of this study demonstrated the importance of tailoring the presentation of systematic overviews to the specific needs and preferences of the study population. It also clearly demonstrated the importance of characteristics of the innovation in the research utilization process. This study also supported the importance of organizational characteristics in the transfer and uptake of research evidence in policy decision-making. The results of this study will assist in the development of future dissemination strategies among public health decisionmakers in public health units. 


\section{Acknowledgements}

I am deeply grateful to Dr. Rhonda Cockerill, my thesis advisor, who provided valuable guidance, expertise, and support throughout my graduate program. Rhonda was always available whenever I needed assistance or reassurance, particularly during the final year of writing this dissertation.

I wish to thank Dr Louise Lemieux-Charles for her ongoing feedback and positive encouragement. Louise introduced me to the concepts of organizational theory and culture, which have now become of great importance to my future research career. Louise not only made herself and her expertise available to me, but also offered many articles and books to assist in my learning. I would also like to thank Dr. Jan Barnsley, who seemed to know just when I needed positive feedback and encouragement. In addition, I am grateful to Jan for stepping in and organizing my defense in order to meet my pressing deadlines. I am also grateful to Dr. Michael Murray who provided many useful recommendations concerning the measurement of variables, and the development of a complex statistical analysis. Michael went beyond my expectations by working closely with me to make the presentation of my analysis as clear as possible.

I would also like to take this opportunity to thank the faculty members of the Graduate Department of Health Administration for providing many challenging and interesting learning opportunities from a multidisciplinary perspective.

I am greatly indebted to Dr. Donna Ciliska who has acted as my mentor for the last seven years. Donna's constant positive feedback as well as expertise in the field, has contributed immensely to this dissertation. I would also like to thank Helen Thomas and Dr. Alba DiCenso, who provided many helpful insights along the way. As well, to all the members of the Public Health Effectiveness Project, Ginny, Sarah, Sheila. Mary Ann, Elena, and Barb, I am thankful for your support and encouragement throughout my graduate program.

I am also grateful to the Hamilton-Wentworth Social and Public Health Services, who not only encouraged and supported me throughout the program, but also provided the necessary resources to make my study possible. This research could not have been completed with out the valuable 
feedback provided by the many decision-makers in this organization. As well, I am indebted to the many decision-makers who took part in the development and testing of my questionnaires. Finally, I am thankful to the many front line staff: public health nurses, environmental health promoters, and healthy lifestyle promoters who wished me well, and inquired on a regular basis about my progress.

I am indebted to the Central East Health Information Partnership who provided me with much needed funding to get my study off the ground. Also I would like to thank Dr. John McGurran for his feedback on the early draft of my dissertation and his strong support of my study.

I am deeply grateful to my family for their love and support. They celebrated each step of this journey with me and were there to comfort me during the more difficult times. Also, a special thank you to Mike, who walked by my side from day one, always demonstrating his confidence in me that I would successfully accomplish this goal. Four years ago Mike told me he hoped he would be able to provide the support I might need to complete this endeavor. All I can say is that I could not have done this without him and his support and love went well beyond what I ever could have imagined possible.

I am sincerely grateful to my fellow students, especially Ruth, Wendy, Maria, Diane, Julie and Lane for helping me to maintain my motivation and confidence, and for many hours of great conversation. We will share a bond for many years to come, that only comes with working through such an overwhelming project. I would also like to give special thanks to Wendy Ungar who spent many hours helping a new student to feel comfortable in this graduate program, and who offered wonderful advice on everything from courses to surviving biostats.

I would also like to thank my friends who have supported me throughout these four years. I am especially grateful to ZsuZsi, who has encouraged me to fulfill my dreams, and whose family has given me many laughs and happy memories over the years. Whenever I needed a break from my graduate world, ZsuZsi's door was always open, with the sounds of children and much laughter providing me with the strength to keep going. 
I am extremely grateful to have received financial support for my doctoral studies from Health Canada through a National Health Research and Development Program (NHRDP) Research Training Award.

Finally, I am grateful to all the public health decision-makers across Ontario who participated in this study and who provided positive feedback and encouragement along the way. 


\section{Table of Contents}

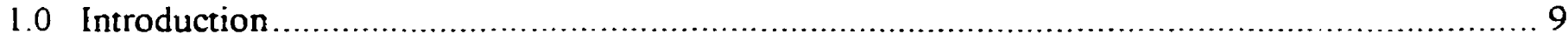

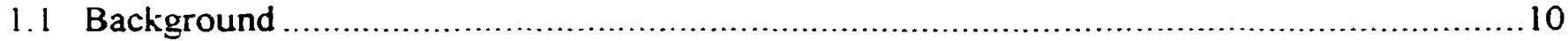

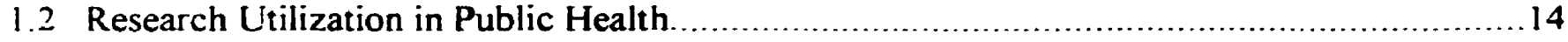

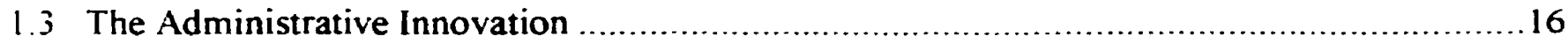

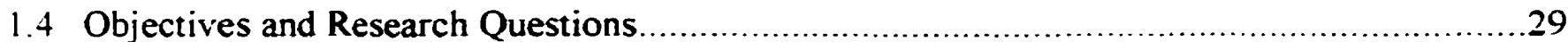

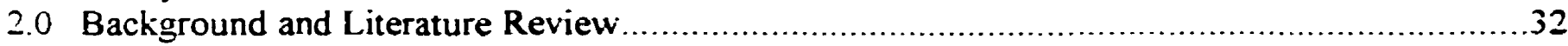

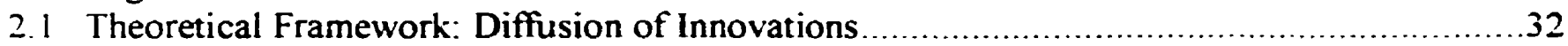

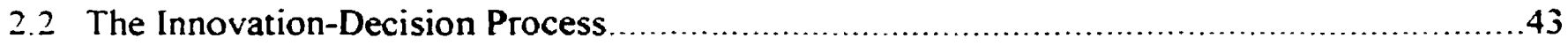

2.3 Diffusion of Innovations in the Health Sector ...................................................4

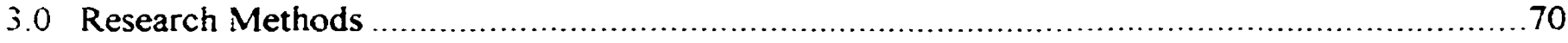

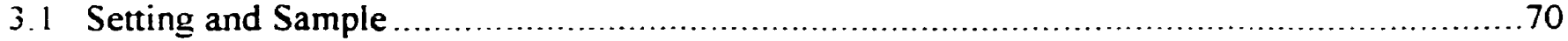

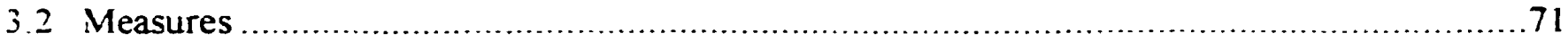

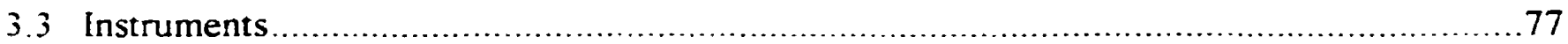

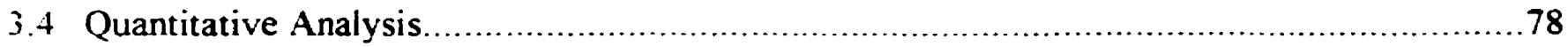

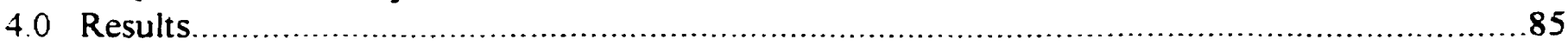

4.1 Characteristics of the Respondents and Public Health Units ..................................85

4.2 Bivariate Correlation Analysis: Dependent and Independent Variables .........................96

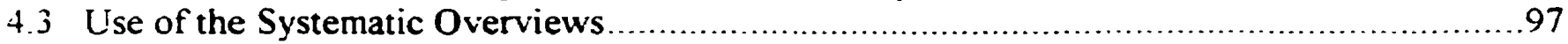

4.4 The Influence of Systematic Overviews on Program Planning .............................. 101

4.5 Influence of Systematic Overviews on Program Justification ................................. 105

4.6 Influence of Systematic Overviews on Program Evaluation ....................................... 110

4.7 Influence of Systematic Overviews on Policy Development Decision ..........................112

4.8 Influence of Systematic Overviews on Staff Development Decisions ..........................115

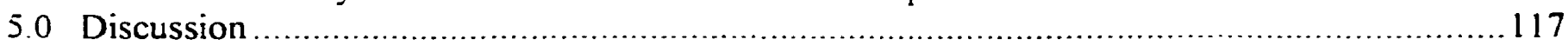

5.1 Impact of the Systematic Overviews Among Decision-Makers ............................ 119

5.2 Implications for Facilitating the Utilization of Systematic Overviews .......................... 139

5.3 Recommendations for Future Research .......................................................... 149

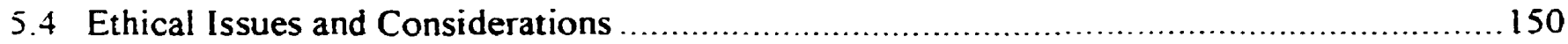

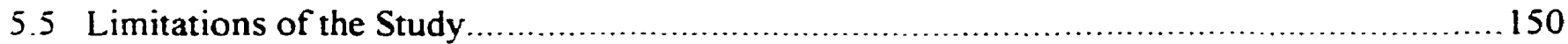

6.0 Conclusions

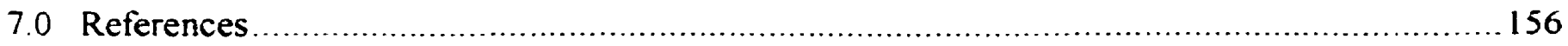

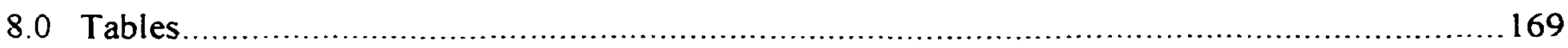

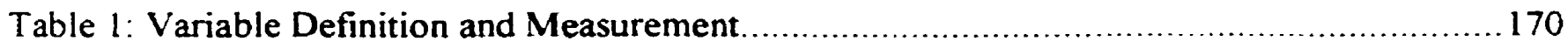

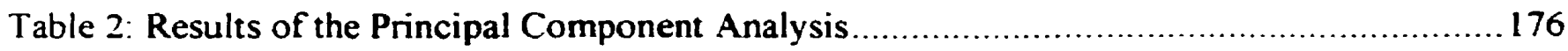

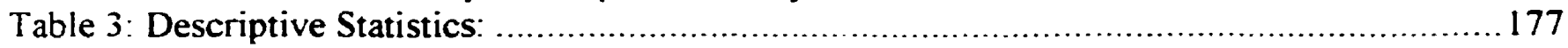

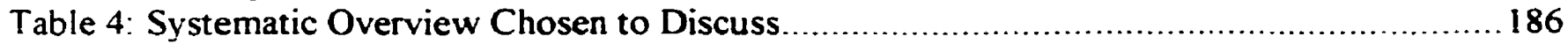

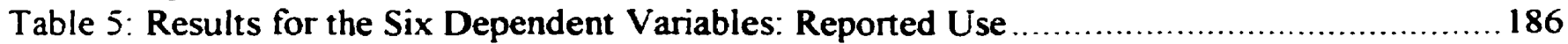

Table 6: Use of Systematic Overview by Position....................................................... 187

Table 7: Influence of Systematic Overviews on Public Health Decisions ............................. 187

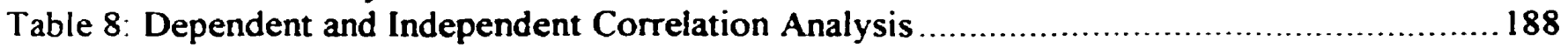

Table 9: Analyses Of Variance - Overall Use of the Systematic Overviews ......................... 190

Table 10:Correlation Coefficients: Overall Use ..................................................... 191

Table 11: Multiple Logistic Regression Analysis: Goodness of Fit Measures....................... 191 
Table 12: Final Logistic Regression Analysis - Overall Use

Table 13: Influence of Systematic Overviews on Program Planning: Analysis of Variance.

Table 14: Correlation Coefficients: - Influence on Program Planning Decisions

Table 15: Program Planning Multiple Linear Regression Analysis...

Table 16: Influence of Systematic Overviews on Program Justification: Results of Analysis of

Variance:

Table 17: Bivariate Correlation Coefficients - Influence on Program Justification................... 196

Table 18: Program Justification Multiple Linear Regression.

Table 19: Influence of Systematic Overviews on Program Evaluation: Results of Analysis of

Variance

Table 20: Bivariate Correlation Coefficients- Influence on Program Evaluation.

Table 21: Program Evaluation Multiple Linear Regression

Table 22: Influence of Systematic Overviews on Policy Development: Results of Analysis of

Variance:

Table 23: Bivariate Correlation Coefficients - Influence on Policy Development .

Table 24: Policy Development Multiple Linear Regression Analysis

Table 25: Influence of Systematic Overviews on Staff Development: Results of Analysis of

Variance

Table 26: Bivariate Correlation Coefficients - Influence on Staff Development ....................202

Table 27: Staff Development Linear Regression Analysis.........................................202

Table 28: Summary of Significant Predictors of all Outcomes: By Outcome .......................203

Table 29: Summary of Significant Types of Characteristics of all Outcomes .......................204

Table 30: Summary of Significant Predictors of all Outcomes: By Variable ...........................205

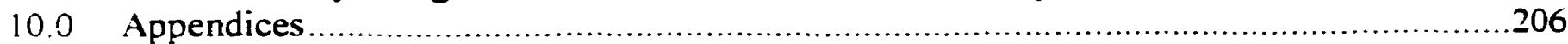

Appendix 1: 1998 Research Utilization Questionnaire .............................................207

Appendix 2: 1998 Organizational Demographics Questionnaire ...............................212

Appendix 3: Ontario Health Care Evaluation Network: Baseline Survey 1996..................213

Appendix 4: Ontario Health Care Evaluation Network: Follow-up Survey $1996 \ldots \ldots \ldots \ldots \ldots \ldots \ldots .215$

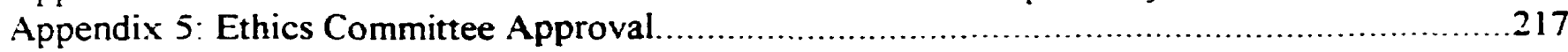

Appendix 6: Information Letter and Consent for Pre-Test Group...................................218

Appendix 7: Introduction and Consent form for Public Health Department Participation ...........220

Appendix 8: Introduction Letter and Consent Form for Public Health Decision-Makers ............222 


\subsection{Introduction}

"Transforming research into practice is a demanding task requiring inteliectual rigor and discipline as well as creativity, clinical judgment and skill, and organizational savy and endurance" (Kitson et al., 1996). Dissemination research, defined as the study of the processes and variabies which determine and/or influence the adoption of knowledge, interventions and practice by various stakeholders (Johnson et al., 1996), has become a priority among health services planners, decision-makers and researchers over the last decade. Since the mid 1980 s there has been an increased emphasis on research examining the relationship between the dissemination of scientific evidence and its use in clinical practice and health care policy decision-making. This dissertation discusses the many factors believed to be associated with research utilization by public health decision-makers in Ontario. The purpose of this study is to examine the association between the adoption/utilization of an administrative innovation, the characteristics of this innovation, as well as characteristics of the organization. environment and the individual. The study sample inciudes decision-makers from Ontario public health units and the innovation is systematic overviews of the effectiveness of five public health interventions. Multiple logistic and linear regression procedures were used to identify which factors of the innovation, organization, environment and the individual significantly predicted the overall use of this innovation and its influence on key public health decisions related to program planning, program justification, program evaluation, policy development and staff development. The results of this dissertation will assist in the development of dissemination strategies for systematic overviews among public health decision-makers across Ontario. 


\subsection{Background}

The goal of research dissemination is to provide information to make decisions about clinical practice and policy. It is a complex task that continues to elude even the most successful dissemination researchers, policy makers and program decision-makers. Several forces such as the growth of science and technology, increased media attention on scientific discoveries, the Internet and the demand for political accountability for the use of public resources. have intensified the need for better dissemination and use of scientific information in health care settings (Johnson et al., 1996). Recent work from the UK predicts that as pressure on resources increases, there will be a necessary transition from opinion-based decision-making to evidencebased decision-making (Gray, 1997; Lomas, 1997). There is also substantial evidence indicating major gaps in the clinical area between the existing evidence and actual practice (Power et al., 1994: Cavanagh et al., 1993; Kenny, 1997). For example, a survey of 1200 nurses reported that only $21.0 \%$ had implemented an intervention based on research evidence in the past six months (Bostrum et al., 1993). In addition, although some of the utilization research has included a variety of factors, the majority of research conducted thus far in health care settings has focused on characteristics of individuals rather than organizational, environmental and innovation characteristics (Rodgers, 1994). Despite considerable effor on the part of dissemination researchers, there remains much confusion on how best to disseminate the 'evidence' to decision-makers, policy makers and practitioners so as to promote its routine use in clinical practice and health policy decision-making.

\section{Definitions of Key Variables}

It is necessary to define key concepts such as diffusion, dissemination, research utilization. evidence-based decision-making and systematic overviews. 
Diffusion refers to the unplanned and uncontrolled process of the spread and adoption of new ideas, techniques, behaviours or products throughout a population (Davis et al., 1997; Ferrence, 1996: Lomas, 1993a).

Dissemination has been defined as the planned or systematic process of communicating new or existing knowledge to a specific audience (Maclean, 1996; Basch et al., 1986; Cameron et al., 1996; Davis et al., 1997; Jennett et al., 1996; Lomas, 1993a).

Research utilization refers to the process of transferring research-based knowledge into clinical practice. (Hunt, 1996; Champion et al., 1989; Gennaro, 1994; Goode et al., 1991; Pettengill et al., 1994; Rodgers, 1994).

Evidence-based decision-making refers to the use of the best possible evidence concerning effectiveness and its application in 'real life' circumstances to make decisions about health professional practices and policies (Hayward et al., 1996a; Birch, 1997; Nutbeam, 1996; Sackett et al., 1996)

Systematic overview is a systematic approach to retrieving and appraising all of the available literature on a research topic to determine the overall effectiveness of a given intervention on specified outcomes, without calculating an overall effect size (Last, 1995; Conn et al., 1996).

Examination of the research utilization literature in the health care field demonstrates that in the majority of studies utilization is measured following the diffusion, rather than the dissemination, of research evidence. It is important to note that the term evidence is composed of many pieces, one of which is research evidence. Currently, clinical practice is determined by a number of distinct pieces of evidence including, 1) past experiences, 2) beliefs, 3) values, 4) skills, 5) resources, 6) legislation, 7) protocols, 8) patient preferences and 9) research results (Estabrooks, 1998; Sibbald et al., 1997). However, some researchers remain convinced that 
practice continues to be predominantly based on experience rather than research-based knowledge (Luker et al., 1992; Baessler et al., 1994; Bohannon et al., 1986; MacPhail, 1997; Limluf et al.. 1992).

The study of research utilization in the health care field has traditionally been limited to measuring the use of scientific research evidence in clinical practice, and in determining which factors inhibit or facilitate its use. More than two decades of research has resulted in the accumulation of a large body of literature concerning the many factors that influence the adoption of research evidence. However, this magnitude of evidence has failed to conclusively identify the most influential factors associated with research utilization for all health care settings, decision-makers, policy-makers and health care professionals. In fact, the literature suggests that there are unexplained differences between health care settings and professionals that require further examination (Royle et al., 1996).

Despite numerous studies, the relationship between research evidence and research utilization in health care organizations remains ambiguous. It has been suggested that there is a continuum that proceeds from knowledge generation to knowledge acquisition and knowledge utilization (Patel, 1996), and that there are several factors which can intercede along the pathway that may facilitate or hinder the attainment of research utilization. Among these factors are beliefs. values, education, social status, and networks (Haines et al., 1994). The process by which characteristics of the innovation, organization, environment and the individual influence research utilization forms the basis of the theoretical framework discussed in this dissertation. The diffusion literature has demonstrated that in some instances it has taken years before new findings were incorporated into practice, while in others, innovations have spread rapidly before evidence of their effectiveness and safety was known (Sibbald et al., 1997). It is these different 
outcomes that are of importance in understanding the relationship between the availability of research evidence, its diffusion and dissemination and whether it is used in practice and policy making.

Research-based clinical change requires more than simply acquiring knowledge (Cavanagh et al., 1996). This thought is strongly supported in the research utilization literature, which indicates that even though health professionals may be knowledgeable and even harbor positive attitudes toward research findings, these attitudes have not been found to be highly correlated with actual use. The dissemination literature suggests that clinical change is brought about when well planned strategies are used to communicate new or existing knowledge to the intended users (Maclean. 1996; Cameron et al., 1996; Lomas, 1993a). Many of these strategies focus on getting the information to the intended users through a variety of techniques. However, considerably less attention has been paid to transmitting the information in a form that can be easily incorporated into practice. One of the major challenges identified by nursing administrators, educators and clinicians has been facilitating the smooth and effective transfer of knowledge from research journals to the clinical areas (Ashcroft et al., 1994).

Research utilization fills the gap between the dissemination of information and its use in clinical practice or program decision-making. It suggests that there are important steps that occur within individuals, organizations and environments that determine whether research evidence will be used and also suggests that characteristics of the research evidence itself have a significant impact on if and how research evidence will be used. The results of decades of research demonstrates the importance of tailoring research evidence to the needs of the target audience, as well as addressing the implications of the new practice or decision on patient outcomes and/or organizational performance (Lomas, 1993a; Dobbins et al., 1998). Thirty years 
ago it was reported that health care professionals often found it difficult to see the practical implications of research evidence (Halpert, 1966). Little has changed in this regard as health professionals continue to rate research applicability to practice as being very low (Funk et al., 1995: Kenny, 1997).

\subsection{Research Utilization in Public Health}

There are only three known studies that have been conducted in the public health setting that address research utilization or the adoption of innovations. Two of these studies are well known in the diffusion literature, were conducted almost thirty years ago and are described below (Becker, 1970a; Becker, 1970b; Kaluzny \& Veney 1973). The third study is less known, was conducted only three years ago, and is discussed in detail in the next section. The first two studies deal with the adoption of innovations and the factors associated with adoption, while the third study identifies barriers to using systematic overviews by public health public health decision-maker. All three studies are similar because they examine the use of research evidence for public health decision-making and identify factors that are associated with its use.

The focus of the Becker study $(1970 \mathrm{a} ; 1970 \mathrm{~b})$ was the identification of factors that were significantly associated with the adoption of innovations. In two separately published reports, Becker determined the factors associated with the adoption of two new public health interventions among medical officers of health. Becker (1970a; 1970b) used a cross-sectional survey designed to examine associations between the adoption behaviours of medical officers of health from heaith units in three states and characteristics thought to be associated with adoption behaviour. The independent variables included measures of centrality (communication networks the participant belonged to), most used sources of information, age, year of graduation, years in current position, standing in medical school graduating class and number of out of state meetings 
attended per year. The introduction of two public health programs, one which was considered to be of high adoptive potential and the other of low adoption potential, were the innovations examined in this study. The program with high adoptive potential was a measles immunization program while the low adoptive potential program was diabetes screening.

Using a telephone survey, Becker (1970a) measured the time of adoption among medical officers of health and then identified which independent variables were associated with time of adoption. Becker found that different attributes were associated with the different public health innovations. For example, early adopters of the measles immunization program. (high adoptive potential), were younger, graduated more recently from medical school, had been in their current positions a shorter time than those who were late adopters and attended more meetings and conferences outside of their local agency than late adopters. However, the reverse was found for the low adoptive potential innovation, which was the diabetes screening program. The early adopters of this program tended to be substantially older, had graduated from medical school many years ago, had been in their current position for many years and had limited contact with the external environment. The results of this study demonstrated that different characteristics of the medical officer of health were associated with the adoption of the two types of innovations (high adoptive versus low adoptive).

The second study by Kaluzny \& Veney (1973) also used a cross sectional survey design to compare characteristics associated with innovation in hospitals and health units. The high response rates indicated that the responding hospital and health units were representative of hospitals and health units in the target population. The mailed survey collected data on attributes of the innovation such as initial cost, continuing cost, rate of cost recovery, payoff, social approval, feasibility of pilot testing the innovation, ease of explaining or understanding the 
innovation, visibility of results, degree to which adopting the innovation is related to the preventive services offered by the health unit and the degree to which the provision of the innovation requires other changes in the health unit. Although the factors examined in this study resulted in a large amount of the variation in innovation in hospitals and health units, $58.0 \%$ and $50.0 \%$ respectively being explained, the overall results demonstrated that different factors were associated with innovation in hospitals when compared to health units.

For example, Kaluzny \& Veney (1973) reported that the factors associated with innovation in health units included being able to pilot test the innovation prior to large scale implementation, the degree to which the innovation was compatible with existing preventive services and the degree to which the innovation had a negative impact on the overall comprehensiveness of the services provided by the health unit. However, factors associated with innovation in hospitals included high payoff, low rate of cost recovery, and low social approval. The results of these two studies demonstrated that innovation among health care professionals varied not only by the type of innovation, such as high adoptive versus low adoptive potential, but also by the health care setting.

\subsection{The Administrative Innovation}

The research utilization literature suggests that there are many factors that act as barriers to health care practitioners and decision-makers in using research evidence in their daily work. One of the main barriers recently identified involves the synthesis of research evidence. Some of the reasons given for why research synthesis is an important barrier are it is very resource intensive and many health care professionals do not have the skills and time to comprehensively appraise and interpret the available evidence (Cameron et al., 1996; Ciliska et al., 1999). 
One strategy that has been suggested among diffusion and utilization experts is for some organization to bring the many sources of information and evidence together and make recommendations for practice, which can then be disseminated to the target audience (Battista, 1989). There has been growing support of the use of systematic overviews and meta-analysis for health care decision-making over the last decade, as the relationship between their use and improved patient outcomes has become known. For example, one meta-analysis of nursing interventions found that patients who received research-based nursing interventions obtained $28.0 \%$ better outcomes than those who received non research-based care (Heater et al., 1988). Another systematic overview of the impact of clinical practice guidelines on patient outcomes in primary care demonstrated that five of the thirteen studies included in the review showed significant improvements in outcomes as a result of the use of the guidelines (Worrall et al., 1997). Other studies have indicated that systematic overviews of research are needed to cope with the increasing volume of research and to avoid the dissemination of conflicting research findings (Angell et al., 1994; Bero et al., 1997; Ganong. 1987).

As a way to reduce or eliminate the barrier of synthesizing research evidence among public health decision-makers in Ontario, the Public Health Effectiveness Project (PHEP) was initiated in 1992. PHEP was originally jointly funded by the Ontario Ministry of Health, Public Health Branch, and the Community Health Nurses' Interest Group of the Registered Nurses' Association of Ontario. The aim of PHEP was to utilize an unbiased, non-politically motivated group of researchers to systematically assess the literature for the effectiveness of public health interventions, and to provide recommendations for practice to practitioners, decision-makers and policy makers. 
There are two types of reports that can be produced when one systematically reviews the literature in a specific topic area, to answer a specific research question, and make recommendations for future practice and research. The first is known as a meta-analysis, which uses a systematic approach to appraise the literature, and then uses the best available evidence to statistically combine the results to produce an overall effect size for a given outcome by a given intervention/service (Sackett et al., 1996). The second type of report is known as a systematic overview, which uses all of the same procedures described above for a meta-analysis except for the statistical computation of one effect size for the given outcome. As opposed to statistically combining the results of all the studies, the objective of a systematic overview is to summarize the findings into meaningful conclusions for all of the outcomes and interventions presented in the literature (Sackett et al., 1996).

Many factors determine the appropriateness of conducting a meta-analysis or a systematic overview. For example, in order for a meta-analysis to have meaningful results, the intervention being examined must be the same across all of the studies, the outcome of interest must not only be the same across studies, but also measured in the same way, and the participants must be the same or at least comparable (Eysenck. 1994). Assessment of public health evaluation studies illustrates that very few studies have similar enough interventions and outcomes to warrant being statistically combined. In addition there is considerable variation in the way in which the outcomes have been measured. Therefore, from a policy-making perspective it was more appropriate for PHEP to conduct systematic overviews as opposed to meta-analyses.

The project was situated within the Hamilton-Wentworth Department of Public Health Services - A Teaching Health Unit affiliated with McMaster University and the University of 
Guelph, with project staff consisting of faculty members from McMaster University who were cross-appointed with the health unit. The original internal steering committee consisted of three faculty members from McMaster University's Faculty of Health Sciences, who had an interest in systematic overviews, the project coordinator who had been a practicing public health nurse. and two program directors from the Nursing and Teaching Health Unit divisions. The external advisory board, which provided feedback to PHEP in terms of future directions, objectives and public health interventions in need of review, consisted of directors of Nursing from other health units in Ontario, as well, as representatives from the two funding bodies discussed above.

The first mission and objective of the Public Health Effectiveness Project was to develop a method for assessing research evidence of the effectiveness of public health interventions. The steering committee, in consultation with the advisory board and other researchers, decided that systematic overviews would provide the most reliable and valid methods for evaluating the effectiveness of public health interventions. Although the members of PHEP were open to conducting meta-analyses. there was an understanding that the majority of public health literature could not be included in meta-analyses due to research design and outcome measurement issues as discussed above. Therefore, the ensuing discussion will focus on the process developed for conducting systematic overviews.

Close affiliations with the Canadian Cochrane Centre ensured the development of methods for conducting systematic overviews that were consistent with those developed previously by the Cochrane Collaboration, an international collaboration started in the early 1970's to examine the effectiveness of medical interventions (Sackett et al., 1996). The process included the development of a comprehensive search strategy resulting in the retrieval of all published and unpublished literature in a given topic area, the development of tools that would 
determine the relevance and validity of the study, and methods for extracting data from the study. All aspects of this process were pilot tested and adapted as needed, during the first two years of the PHEP.

During these two years, consultation with the external advisory board resulted in the identification of five public health interventions that were perceived to be of high priority requiring recommendations for practice. This included interventions related to home visiting by public health nurses, parent-child health, heart health promotion, adolescent suicide prevention and community development. By May 1996, five systematic overviews related to these topics had been completed by PHEP. In all cases one of the three cross-appointed faculty members from the Faculty of Health Sciences at McMaster University, or the project coordinator took the lead role in conducting the systematic overview. In most cases the lead author also had expertise in that topic area which facilitated the development of appropriate research questions.

The five systematic overviews completed during this three year period were: The Effectiveness of Home Visiting as a Public Health Intervention (Ciliska et al., 1996), The Effectiveness of Adolescent Suicide Prevention Programs (Ploeg et al., 1995a), the Effectiveness of Community-Based Heart Health Projects (Dobbins et al., 1996), The Systematic Overviews of the Effectiveness of Community Development Projects (Ploeg et al., 1995b) and a Review of Reviews of the Effectiveness of Parent-Child Interventions (Hayward et al., 1996b).

The systematic overview process consisted of seven distinct steps including the identification of a research question, the development of a comprehensive search strategy, the retrieval of potentially relevant studies, the assessment of all studies for relevance, the assessment of all relevant studies for methodological rigor, the extraction of data from the most rigorous studies and the synthesis of the data into recommendations for practice. For each of the 
five public health interventions studied, a relevant and appropriate research question was identified by briefly looking at the literature in that area and asking practitioners in the field what would be useful information for them in their daily practice. Following the development of the research question, a comprehensive search strategy that included both published and unpublished literature was developed. Once all of the studies identified in the search strategy were retrieved, each study was assessed for relevance to the research question, and those deemed relevant were then assessed for methodological quality (validity). Relevance and validity assessments were initially conducted on a subset of twenty articles by two independent reviewers, and if there was a high level of inter-rater agreement on these studies, then only the primary author assessed the remainder of the retrieved articles for relevance and validity. The acceptable level of inter-rater agreement to warrant only one reviewer was set high at a Kappa of 0.80 , which was considered to be rigorous among members of the Cochrane Collaboration (Sackett et al., 1996). If there were any discrepancies in ratings between the two independent reviewers on the initial subset of studies they met to discuss and resolve them before moving on to the extraction of data.

The purpose of rating the studies for validity was to determine the methodological rigor of the study design and to classify articles as being 'strong', 'moderate' or 'weak'. In all five overviews the 'strongest' available evidence was used to develop recommendations for practice. Pertinent data on the outcomes of interest, independent variables and specifics of the intervention itself were then extracted by two independent reviewers. These data were then used to make recommendations for public health practice in the form of a working document. Several drafts of the working document were circulated to a variety of public health decision-makers for comment and feedback prior to being made available to the larger audience. 
Once these five systematic overviews were completed, PHEP was faced with the task of disseminating these overviews to public health practitioners, decision-makers and policy makers in Ontario. The project team was concerned with disseminating the overviews in a manner that would facilitate their use. A review of the literature demonstrated that although considerable research in the area of dissemination and utilization existed, very little was known about how best to disseminate and facilitate the use of research evidence, particularly systematic overviews, among public health decision-makers. In addition to there being little evidence concerning effective methods for disseminating systematic overviews in general, there was no available research reporting effective dissemination techniques among public health decision-makers in Ontario or Canada. As the project team attempted to develop a dissemination strategy, it became evident that more information was needed from public health decision-makers concerning the barriers and facilitators of research utilization and their perceptions of how systematic overviews could be used for program decision-making. Therefore the project team developed a new objective, to develop an effective dissemination strategy and to examine how the utilization of systematic overviews among public health decision-makers could be facilitated. This new objective led to the development of a research proposal that was later funded by the Ontario Health Care Evaluation Network (OHCEN) in 1996.

Before continuing with a description of the OHCEN study, it will be useful to illustrate the series of research studies, one of which is this doctoral dissertation, that have occurred since 1996 when the OHCEN study was funded. 
Figure 1: Time Sequence of the OHCEN Study and the Study for this Dissertation

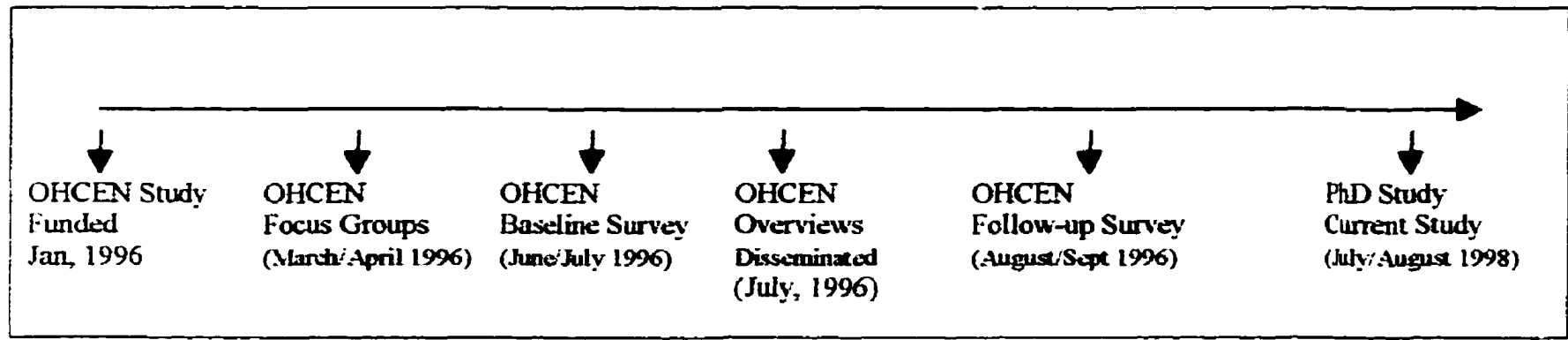

As this diagram demonstrates the OHCEN study was conducted during the summer of 1996 and consisted of focus groups and a baseline and follow-up survey conducted three months a part. The second study, which is this doctoral study, was conducted two years after the initial study during the summer of 1998 .

The purpose of the descriptive OHCEN study was to better understand the research needs, perceptions of barriers to research utilization and attitudes towards systematic overviews of decision-makers in public health at the level of health systems planning. This included program managers, program directors, associate medical officers of health, medical officers of health, and consultants with the Ministry of Health, Public Health Branch. Specific objectives of the study included: identifying the target audience for the dissemination of systematic overviews: assessing decision-makers' awareness of and attitudes towards systematic overviews as a method of research transfer; assessing the current relevance and validity of available overviews to decision-makers; providing access to systematic overviews in different formats; and identifying factors influencing the utilization of systematic overviews in public health policy decisions (Ciliska et al., 1999). The OHCEN study consisted of the following four stages: conducting focus groups to assist in the development of a telephone survey tool; administering the baseline 
telephone survey; disseminating the five systematic overviews to all study respondents; and administering the follow-up telephone survey (Ciliska et al., 1999).

The focus of the baseline telephone survey was to collect data on respondents' perceptions of the barriers to using research evidence in program decision-making, to assess their understanding and awareness of the availability of systematic overviews related to public health interventions, and to assess their perceptions as to the usefulness of systematic overviews. The telephone surveys were developed in part by reviewing the relevant literature such as research utilization and dissemination and the diffusion of innovations. The input from two focus groups consisting of 8-10 senior public health decision-makers or administrators was also used in finalizing the surveys. The focus groups were asked to explore issues related to research access and utilization of systematic overviews (Ciliska et al., 1999). These focus groups were also asked to comment on how systematic overviews could be used in program decision-making, which formed the basis of the outcome variables measured in this doctoral study. The focus groups suggested that systematic overviews could possibly have an impact on a variety of public health decisions including those related to program planning, program justification, program evaluation, policy development and staff development. The questionnaire was then pre-tested for content validity and verbal comprehension, then revised and tested for test-retest reliability. Any questions with a test-retest correlation coefficient below 0.5 were removed from the survey.

Since the number of potential respondents was small, all of the decision-makers who met the inclusion criteria, currently working in public health in Ontario, and making decisions related to public health practice, were approached to participate in the study. The sampling frame was developed by contacting each public health unit in Ontario and the Public Health Branch and 
obtaining the names of all managers, directors, associate medical officers of health, medical officer of health, and consultants who met the above named criteria.

Two hundred and seventy decision-makers were asked to participate in the baseline telephone survey, of whom $87.0 \%$ agreed to participate. During the initial telephone survey the following data were collected: individual characteristics related to age, discipline, date of graduation, years in public health and years in current position. Respondents were also asked to comment on their perceptions of barriers to using research evidence in decision-making. This included barriers related to lack of decision-making authority, lack of critical appraisal skilis, lack of resources, lack of time, lack of relevance of research to decisions and unsupportive work environments. Finally, respondents were asked whether they believed systematic overviews could overcome these barriers.

At the end of each baseline telephone interview, each respondent was made aware of the five systematic overviews and asked if they were interested in receiving any of them. An individualized package of requested systematic overviews was then sent to each respondent. The systematic overviews were available as full working papers published through the Quality of Nursing Worklife Research Unit, one-page abstracts, two-page executive summaries and the full working paper on the Internet. Approximately $95.0 \%$ of the respondents requested at least one systematic overview with the majority requesting two or more. The most popular requested format for the overviews was as the full working paper published by the Quality of Nursing Worklife Research Unit.

Three months after receiving the systematic overviews all the respondents were contacted again for a fifteen minute follow-up telephone interview, which was completed during August/September 1996. During this follow-up interview respondents were asked about receipt 
of the overviews, if the overviews had been read, perceived usability, relevance and application; and further dissemination of the reviews. Respondents were also asked to comment again on whether they perceived systematic overviews could overcome the identified barriers to using research evidence in public health decision-making. The response rate for the follow-up interview was also high at $93.0 \%$. Therefore, data for both the initial telephone survey and the follow-up survey were available for 225 public health decision-makers.

Lising this before/after survey design, Ciliska et al (1999) found that $68.8 \%$ of respondents perceived time (not having enough time to search, read and synthesize research) to be the greatest barrier to using research to make evidence-based decisions. Respondents also perceived not having the resources to put research results into practice $(48.6 \%)$, not being able to get research information fast enough to include it in the decision-making process (33.7\%), and having difficulty in changing the existing approach or program $(23.8 \%)$ as being serious to very serious barriers to using research (Ciliska et al., 1999). These results were similar to an earlier research utilization study conducted among public health nurses which found that heavy workload, research findings that were not transferable to practice, limited authority to implement new ideas, insufficient time to implement new ideas, and not enough time to read research, were important barriers to using research in practice (Stolk et al., 1995).

Ciliska et al (1999) also found that respondent's perceptions about the barriers to using research in decision-making decreased after receiving one or more systematic overviews. The majority of respondents, $78.7 \%$, believed that a systematic overview disseminated to individual public health decision-makers, possibly or definitely would decrease the barrier of not having enough time to synthesize the evidence. A total of $81.0 \%$ of respondents recalled receiving an overview, and of those $71.0 \%$ had read or skimmed it within three months of receiving it, and 
approximately $30.0 \%$ stated that the systematic overview had played a part in program planning or decision-making at that time. As well, $67.0 \%$ stated they expected to use at least one of the systematic overviews in the future.

Although there were many interesting and enlightening findings from this study, there were still many unanswered questions with respect to the factors that facilitated the use of systematic overviews in program decision-making, and which factors distinguished those decision-makers who utilized systematic overviews from those who did not. For example, the results of the chi-square analysis conductd by Ciliska et al (1999) revealed no significant differences in reported use by any of the individual characteristics such as age, discipline, years since graduation, position, or years in current practice. Therefore, although there were differences in reported use among public health decision-makers, the factors measured in the OHCEN study failed to distinguish between users and non-users. The study did however demonstrate that public health decision-makers judged themselves as having a high need for research information, that this need was not currently being met, and that, in general, they had positive attitudes toward the usefulness of systematic overviews (Ciliska et al., 1999).

Lipon the completion of this study, PHEP was still faced with the task of identifying factors that would facilitate the utilization of systematic overviews among public health decisionmakers, and developing an effective dissemination strategy. During the OHCEN study, PHEP's project coordinator enrolled in a doctoral program in Health Administration, and began developing a PhD study that would expand on the work of Ciliska et al (1999). A more indepth review of the diffusion of innovations literature as well as the research dissemination and utilization literature, demonstrated that organizational and environmental characteristics were increasingly being discussed as important factors in the adoption of innovations and the 
utilization of research evidence. (Dobbins et al., 1998). The most recent findings from this literature also indicated that characteristics of the innovation, organization and the environment were significantly associated with the use of research evidence for policy making. Since organizational and environmental characteristics had not been measured in the OHCEN study, there continued to be no available data as to the impact of these variables on public health decision-makers, with respect to research utilization. In addition the study design employed in the OHCEN study did not lend itself well to advanced statistical procedures such as logistic and linear regression analysis, and therefore no analyses beyond descriptive analyses and t-tests were conducted on the measured characteristics of the innovation and the individual. It was clear that there were important data available from the OHCEN study that could be used in a follow-up study. Also a follow-up study would provide an opportunity to gather data on organizational and environmental characteristics, which had not previously been collected among public health decision-makers in Ontario. Therefore, PHEP's former project coordinator, the author of this dissertation. developed a research utilization study proposal to conduct a follow-up study to the OHCEN study that would be conducted two years after the dissemination of the five systematic overviews. The research proposal was developed independently by this Ph.D student. However, an advisory committee made up of researchers, and the medical officer of health, and director of the Teaching Health Unit at the Hamilton Public Health Unit were consulted to determine the feasibility of the project. The main focus of this second study was to gather new data from the same decision-makers who participated in both of the OHCEN surveys, with respect to their perceptions of their organizations and environments, as well as how they had used the systematic overviews during the past two years. This Ph.D study also used data collected in the OHCEN study on characteristics of the innovation and the individual, such as their perceptions regarding 
the usefulness of the systematic overviews, as well as their perceptions regarding identified barriers to using research evidence in practice. There are some methodological issues with using data that are two years old including perceptions may have changed between the time at which the systematic overviews were disseminated and the time data on their utilization were collected. It may be that as the respondents became more familiar with the systematic overviews their perceptions of their usefulness also improved. This change in perceptions would not have been captured in this study since this data was not collected at the two year follow-up period. However, respondent's perceptions of the systematic overviews at the time at which they received them may have played an important role in whether the decision-makers planned to use the systematic overviews in the future. Therefore, it is important to include these perceptions from the 1996 data, while recognizing that changes in these perceptions, that may have affected utilization. may have occurred during this two year follow-up period.

\subsection{Objectives and Research Questions}

The study of research dissemination and utilization in public health in Ontario is in its infancy with many potential research questions still to be answered. This dissertation focused on continuing to build on the work of Ciliska et al (1999), and Stolk et al (1995), who identified a number of barriers and facilitators to using research evidence in public health practice in Ontario. The research objectives for this dissertation were developed keeping in mind the overall objective of developing future dissemination strategies for systematic overviews among public health decision-makers. The research objectives of this dissertation were:

1. To determine the extent to which public health decision-makers used five systematic overviews of the effectiveness of public health interventions that had been disseminated to them two years earlier. 
2. To determine which characteristics of the innovation, organization, environment and the individual predicted if the five systematic overviews were used among individual public health decision-makers in Ontario.

3. To identify which characteristics of the innovation, organization, environment and the individual predicted whether the five systematic overviews influenced public health decisions related to program planning, program justification, program evaluation, policy development, and staff development, among individual public health decision-makers in Ontario.

Aithough there is considerable literature concerning the associations between the diffusion of innovations/research utilization and characteristics of the innovation, organization, environment and individual, it is unclear if similar associations exist in the public health field. Therefore, it was not feasible or appropriate at this time to test hypotheses about the direction of these associations in this study. Alternatively this dissertation will identify those associations that exist between the dependent and independent variables, as well as give some preliminary evidence of the direction of the associations. The findings from this study will assist in the generation of testable hypotheses in future research dissemination and utilization studies in public health. The findings from the literature review as well as Ciliska et al (1999), and Stolk et al (1995), were used to develop the following specific research questions based on the objectives of this study.

1. What percentage of public health decision-makers reported using the systematic overviews two years after receiving them. (Objective l)

2. Were there significant differences in overall use of the systematic overviews according to the position the decision-maker held at the time the overviews were disseminated. For example a) did program managers and program directors use the systematic overviews significantly more than Medical or Associate Medical Officers of Health. (Objective 2)

3. Which types of characteristics, such as organizational, environmental, individual and characteristics of the innovation were most strongly associated with overall use of the systematic overviews. (Objective 2) 
4. On which types of public health decisions, (program planning, program justification, program planning, policy development, and staff development) did systematic overviews have the greatest influence. (Objective 3) 


\subsection{Background and Literature Review}

There appears, particularly in the health care setting, to be a natural progression from the study of the diffusion of innovations, to the study of the utilization of research evidence to the study of the effectiveness of dissemination strategies in promoting the use of research evidence for decision-making. As such there were several relevant bodies of literature, which provided noteworthy insights into the development of the conceptual framework utilized in this dissertation, including the research dissemination and utilization literature, the evidence-based medicine and decision-making literature and finally the management literature. Those theoretical frameworks considered of prime importance to this dissertation were Rogers' Diffusion of Innovations, organizational theory, and individual and organizational decision-making.

The theoretical constructs within each of these areas have contributed to the understanding of research dissemination and utilization. Although Rogers (1995) dedicated a great deal of effort to identifying categories of adopters such as early and late adopters, the point at which decision-makers adopted the systematic overviews was not the focus of this dissertation and therefore will not be included in the theoretical framework. The focus of this dissertation however, was to identify characteristics that distinguished individual decision-makers that used the systematic overviews to make public health decisions, from those who did not. Therefore, those concepts of the diffusion of innovations theory that assisted in identifying these characteristics were included in the framework and are discussed in detail in this chapter.

\subsection{Theoretical Framework: Diffusion of Innovations}

The limited success of dissemination research in the health care setting has encouraged researchers to look for answers from a variety of theoretical frameworks and bodies of literature. 
Rogers' Diffusion of Innovations theory has been particularly useful, and used extensively in the last twenty years to gain a greater understanding of the forces at work in transferring knowledge to practice. Diffusion scholars have long recognized that an individual's decision about an innovation is not an instantaneous act. Rather it is a process that occurs over time and consists of a series of actions (Rogers, 1983). The diffusion of innovations refers to the spread of new ideas, techniques, behaviours or products throughout a population (Rogers, 1983). Furthermore, the term innovation has been defined as the adoption of a change which is new to an individual, organization or the relevant environment' (Scott, 1990). Another definition states that innovation is 'any idea, practice, or material artifact perceived to be new by the relevant unit of adoption' (Damanpour, 1991; Scott. 1990). Innovations in health care may be preventive, curative, rehabilitative, or palliative, and encompass all of the instruments, equipment, drugs and procedures used in the delivery of health care services (Battista, 1989). These definitions suggest that the adoption of research evidence can be considered an innovation, and it is possible that the factors that affect adoption of innovations in those fields are also applicable to the health care setting.

Battista (1989) further classified medical innovations into high, medium and low technologies. High technologies require major capital investments and mobilization of enormous human, physical and administrative resources, whereas medium technologies can be used without an elaborate and complex support system, and low technologies do not require mobilization of many financial and human resources. When the innovation is a systematic overview of the effectiveness of a public health intervention that is to be used for program decision-making, this innovation represents a low technology. 
The diffusion literature suggested at least two decades ago that there was a substantial time lag of eight to fifteen years between the time technical information was generated and the time it was used in actual practice (Utterback, 1974). Although this time lag is shorter now, time lags still exist. This is problematic in the health care setting where advances in knowledge and technology may be linked to more positive health outcomes that are not being realized due to the under utilization of this knowledge. This finding is also significant in health care given that the creators of research evidence are seldom its users. It is well known that researchers generally write research articles for other researchers, which in many instances makes it difficult to implement the findings in daily practice (Lomas, 1997).

Rogers (1983) determined that the cumulative rate of adoption of innovations is characterized by an S-shaped curve which increases slowly at first, then more rapidly, and finally slows and levels off (Ferrence, 1996; Rogers, 1983). Decisions regarding whether or not to adopt an innovation are essentially "information seeking and information processing activities in which the individual or organization is motivated to reduce uncertainty about the advantages or disadvantages of the innovation" (Rogers, 1983). Rogers conceptualized the innovation process as consisting of five stages, namely, knowledge, persuasion. decision, implementation and confirmation.

The knowledge stage "commences when the individual (or decision-making unit) is exposed to the innovation's existence and gains some understanding of how it functions" (Rogers, 1983). During the persuasion stage of the innovation-decision process, selective perception becomes increasingly important for it is at this stage that attitudes toward the innovation are formed. It is at this point that individual perceptions concerning the innovation's relative advantage, compatibility and complexity are developed (Rogers, 1995). During the 
persuasion stage individuals seek to answer questions such as what are the innovation's consequences, and what are the advantages and disadvantages of its use. This type of information is usually sought from near-peers whose subjective opinion of the innovation (based on their personal experience with adoption of the innovation) is most convincing. Therefore if someone in a similar position or with similar likes and dislikes to oneself indicates a positive experience in adopting an innovation, then motivation to adopt increases (Rogers, 1995).

During the decision stage, individuals engage in action to either adopt or reject the innovation. Diffusion research has demonstrated that innovations that can be divided for trial are generally adopted more rapidly. Methods to facilitate the trial of innovations such as the distribution to clients of free samples, will speed up the rate of adoption (Rogers, 1995). As well, the diffusion literature demonstrated that for some individuals and for some innovations, the trial of a new idea by a person like themselves or an opinion leader, can substitute, at least in part, for their own trials of an innovation (Lomas, 1993c). Therefore, if a colleague tries out the innovation and recommends it, then the individual might forgo the trial period and decide to adopt the innovation fully. Implementation occurs when " an individual puts an innovation into use" (Rogers, 1983), and finally in the confirmation stage the individual seeks reinforcement for the innovation decision already made, but may reverse this decision if exposed to conflicting messages about the innovation" (Rogers, 1983).

Although Rogers has outlined five steps involved in the diffusion of innovations process, only three of these stages were the focus of this dissertation. These included the persuasion, decision and implementation phases. In the public health field the persuasion stage could be operationalized as decision-makers' perceptions of how useful systematic overviews were in making decisions about public health practice. The decision stage could include measures of the 
decision-making process itself and perceptions concerning the routine use of research evidence at both the individual and organizational levels, for decisions related to program planning, program justification, program evaluation, policy development and staff development. The implementation stage could represent the research utilization outcomes, which could be measured as use versus non-use, and the degree to which the systematic overviews influenced the types of public health decisions discussed above. A number of characteristics of the innovation, organization, environment and individual have been identified in the diffusion and research dissemination and utilization literature, as having important effects on each of these three phases of the diffusion process, all of which will be discussed in greater detail later in this chapter.

One misconception of this model is the expectation that innovation adoption will occur along a linear path. However, more and more writers of the diffusion literature emphasize the nonlinear, slightly chaotic, usually sloppy, sometimes random and often up-side down nature of innovation (Scott, 1990). Although it is important to understand the process of innovation adoption in general, one must also be open to observing a variety of patterns during this process. In addition, this framework was developed using the adoption patterns of agriculturat innovations, which may follow a somewhat different path than health care innovations, although the same basic theoretical components may be the same.

Other diffusion researchers have pointed out that increasing turbulence and competitiveness of organizations' environments have made the identification, evaluation, and adoption of innovations a critical determinant of organizational performance (Bigoness et al., 1981). More than twenty years ago the diffusion literature was strewn with research findings that varied from study to study, setting to setting, and innovation to innovation. Diffusion researchers 
at the time pointed out that these variations in findings were important because they demonstrated that the diffusion process was ever changing and suggested that different actors facilitated and hindered the diffusion process depending on the organization and the innovation. (Downs et al.. 1976).

Rogers' extensive study of the diffusion of innovations over the last several decades has resulted in the evolution of a theoretical framework consisting of four discrete elements. These include the innovation, communication channels, time and the social system (Rogers, 1995). Although Rogers' work was used to form the basis of the framework discussed below in Figure 2. some further refinements to the model have been made based on the research dissemination and utilization literature in the health care setting, and discussions with key informants in the public health field. For example, although Figure 2 displays the same number of main elements as discussed by Rogers' (1983), the four elements have been altered slightly to represent characteristics of the innovation, the organization, the environment and the individual. The social system has been redefined and placed within the context of the organizational and environmental elements as suggested by Lewis et al., (1993). The communication channels element has been redefined somewhat as well, and has been incorporated within each of the four elements. Finally the time element was incorporated within the innovation and individual elements by measuring variables both before and after the innovation was disseminated.

Figure 2 illustrates the research utilization framework that was developed and examined in this study, and demonstrates the complex inter-relationships that exist between the four main elements or types of characteristics. For example, the framework depicts that individuals may exert influence over their environments and vice versa. The association among these two 
elements has been supported by Orlandi, (1996), who suggested that individuals have the ability to shape their environments just as environments shape individual behaviour.

Figu re 2: Research Dissemination and Utilization Framework

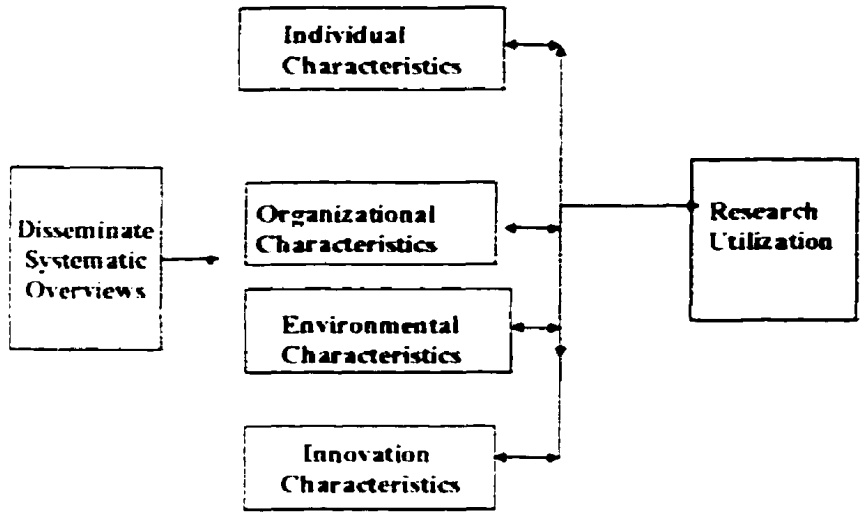

This framework illustrates that the utilization of research evidence, in this case, five systematic overviews, once disseminated among public health decision-makers, is influenced by a variety of characteristics related to the innovation, the organization, the environment and the individual. As well, the process is further complicated by the influence each type of characteristic has on the other characteristics. For example, individual characteristics are influenced by organizational, environmental and perceptions of the innovation, while organizational characteristics are influenced by the remaining characteristics.

Rogers (1995) defined the social system as a set of interrelated units that are engaged in joint problem-solving to accomplish a common goal. This element has been further broken down into the three distinct categories of organizations, environments and individuals (Kaluzny, 1974; Rogers, 1995; Warner, 1975). Each category has a number of inherent characteristics that make them unique from each other and from other decision-making units. These characteristics or social structures as Rogers refers to them, affect how innovations are diffused within different social systems. Other factors that Rogers (1995) suggested impact on the diffusion process 
include organizational norms, roles of opinion leaders and change agents, the types of decisions being made and the consequence of the innovation.

Although much has been learned about the factors associated with the adoption of innovations in the management field, questions remain as to the specific factors that contribute to different adoption behaviours among different groups of individuals and organizations. The health care setting is one example where there has been great disparity in the adoption of innovations, not only between health care organizations, but also health care professionals. Kaluzny \& Veney (1973) and Becker, (1970a, 1970b) demonstrated aimost thirty years ago that the factors associated with innovation adoption were significantly different between public healih practitioners and hospital-based practitioners. Becker (1970a, 1970b) demonstrated that there were significant differences between medical officers of health who adopted or did not adopt innovations, and that the innovation itself also played an important role in predicting who adopted and who did not adopt the innovation. The results of these two early studies demonstrated that the diffusion of innovations was affected by both individual characteristics and characteristics of the innovation. However, ongoing research to determine the effect of organizational characteristics on innovation adoption among public health units was not continued after these initial studies. More recently, interest has grown with respect to measuring and understanding the impact of organizational characteristics on innovation adoption among public health units, as justification for scarce resources from local city councils has become more important.

Rogers (1995) pointed out that organizations have unique ways of communicating and working, and that these structures predict in part, the behaviour of individual members of that social system, including when and if they adopt an innovation. This concept illustrates the 
importance of measuring not only characteristics of the public health decision-maker but also characteristics of the public health unit within which the decision-maker works. As well, since individual health units make decisions within the constructs of their unique environments, then characteristics of the environment are also likely to have an impact on the adoption of innovations, which in this case were the five systematic overviews.

The concept of individual and organizational decision-making has recently become more prevalent within the diffusion of innovations and research utilization literature. As discussed previously, Rogers (1995) described the innovation-decision process as an information-seeking and information-processing activity in which the individual (organization) was motivated to reduce uncertainty about the advantages and disadvantages of the innovation. This concept identified the importance of decision-making both at the individual and organizational level and has led to a number of hypotheses concerning the processes and outcomes of decision-making. For example, the literature demonstrated that different decision-making processes lead to different outcomes with respect to innovation adoption and research utilization (Langley et al., 1995; Mintzberg et al., 1976; Nutt, 1984). Therefore, it became clear that both individual and organizational measures of decision-making were important elements to include within the theoretical framework constructed for this dissertation.

The process by which organizations make decisions has become predominant in several bodies of literature over the last decade. Some researchers would suggest that organizational decision-makers negotiate policies and determine the interest, resources, and strategies which they bring to the decision process (Greer, 1977). This is particularly relevant to public health decision-makers who make decisions within the context of finite resources, and to some extent established policies concerning the provision of services. 
Early work on the diffusion of innovations in the public health sector was conducted by Kaluzny. (1974). In his report, Kaluzny indicated that the role of innovation within public health units was determined by how well the organizations and individuals within those organizations were able to adopt and meet changing environmental demands. He elaborated further by suggesting that as new health problems emerged and changes occurred in expectations, cultural norms and values, and economic and political power. health units and public health professionals were challenged to meet these changing needs. These same types of pressures and demands to meet changing needs continue to impact on public health policy and decision-makers in 1999. Therefore, Kaluzny's (1974) findings remain applicable in the current context, and provide support for the importance of environmental characteristics with respect to innovative adoption. However, it is surprising given these earlier findings that environmental characteristics have not received more attention in the diffusion of innovations and research utilization literature in health care over the last two decades. The public health field in particular may be particularly susceptible to environmental pressures given that it is mandated by provincial and local politicians, as well as administrative bureaucrats.

The innovation-decision process consists of a series of actions and choices over time through which an individual or decision-making unit evaluates a new idea and decides whether or not to incorporate the innovation into ongoing practice (Rogers, 1995). There are many characteristics inherent in individuals, organizations, environments and the innovation itself that determine how this process occurs. One of the factors to dictate this process is related to the values, interests and beliefs of the individual. For example, diffusion research has demonstrated that individuals tend to expose themselves to ideas that are in accordance with their interests, needs and existing attitudes. Also individuals consciously or unconsciously avoid messages that 
are in conflict with their predispositions (Rogers, 1995). Hassinger (1959) suggested that even if individuals are exposed to an innovation message, this exposure will have little effect unless the innovation is perceived as relevant to the individual's needs and as consistent with the individual's attitudes and beliefs. Rogers (1995) identified a number of attributes from the diffusion literature that were associated with individuals becoming aware of innovations at an early stage. Those attributes most applicable to public health decision-makers were having more exposure to mass media channels of communication and being more cosmopolite.

Diffusion researchers have suggested that in order for progress to continue in this field additional work examining attributes of the innovation itself are necessary, before comparing the rate of adoption between organizations and identifying variables that predict the rate of adoption. Damanpour, (1991) identified several different types of innovations which he hypothesized would have a moderating effect on the rate of adoption. Of particular interest to this study was the difference between technological innovations and administrative innovations. Technological innovations were defined as products, services and production process technologies that were related to basic work activities and could concern either product or process. He defined administrative innovations as involving organizational structures and administrative processes that were indirectly related to the basic work activities of an organization and were more directly related to its management. The innovation in this study, which was systematic overviews of the effectiveness of public health interventions, fits more closely with the definition of administrative innovations, and therefore characteristics shown to be significantly associated with these types of innovations were examined in this study. Although the systematic overviews themselves were administrative innovations, implementing new public health practices would be considered to be technological innovations. So as to avoid confusion among respondents as to 
which innovation was being discussed, the survey questions were carefully designed to elicit responses related only to the usefulness of the systematic overviews regardless of the content of the overviews.

Rogers (1995) pointed out that relatively little effort has been devoted to analyzing innovation differences (i.e. how the properties of innovations affect their rate of adoption). Also, particularly in the health care field, only recently have attributes of the innovation begun to be mentioned as important characteristics in need of study. A review of the diffusion literature demonstrated that there were five main attributes of the innovation that explained a significant amount of the variation in adoption rates. These include relative advantage, compatibility, complexity, trialability and observability (Rogers, 1995). Rogers also demonstrated that the receiver's perceptions rather than an expert's perceptions of the attributes of an innovation affected the rate of adoption. Other diffusion researchers indicated that the most appropriate design for determining causality between attributes of the innovation and adoption would result in data on the attributes of the innovation being gathered prior to, or concurrently with, individuals' decisions to adopt the innovation (Tornatzky et al., 1981).

\subsection{The Innovation-Decision Process}

Bigoness et al (1981) identified the two competing types of diffusion studies that have been conducted over the last thirty years. The first type of study addressed the micro issue of internal organizational change and focused on a very specific area of innovation within individual organizations. Although Bigoness et al (1981) agreed that this type of research was very desirable, they suggested that a more macro approach to diffusion research would be more beneficial to advancing the knowledge in the field. This concept, although three decades old, contributed to the research design chosen for this dissertation. Since little research on the 
diffusion of innovations has been conducted in the public health field, a research study focusing on the macro issues of the diffusion of innovations will be of the greatest benefit at this time. Therefore. this study focused on identifying characteristics that differentiated those public health decision-makers who utilized the systematic overviews from those who did not, as well as the factors associated with the systematic overviews influencing decisions related to program planning, program justification, program evaluation, policy development and staff development. It was also believed that a study of the marco issues at this time would facilitate the development of needs specific dissemination strategies.

One of the shortcomings of diffusion research is the pro-innovation bias, which is the idea that an innovation should be diffused and adopted by all members of a social system and that it should be diffused more rapidly than it has been (Rogers, 1995). One of the ways in which the pro-innovation bias becomes included in diffusion research is through the selection of the innovations being studied (Rogers, 1995). Another important criticism of the diffusion research design is that it entails the use of participant recall for data collection. Much research has demonstrated that recall bias occurs frequently. The research designs predominantly used in diffusion research do not provide much information about the process of diffusion over time, other than what can be reconstituted from respondent's recall data. In an attempt to address recall bias. data related to perceptions of the innovation and the individual were collected at the time the innovation was disseminated rather than during the two year follow-up survey.

\subsection{Diffusion of Innovations in the Health Sector}

Although it seems feasible to directly apply the knowledge learned over several decades of diffusion of innovation research to the health care setting, there are those who argue otherwise. Despite the many similarities that would allow such direct application to occur there 
are unique differences between industry-type organizations and health care organizations that may hinder direct application. Some argue that innovations within health care units are increasingly likely to reflect an organizational or even an interorganizational process rather than an individual one, and therefore many of the individual attributes associated with innovation diffusion in industry are not directly applicable to health care organizations (Scott, 1990). The following section will outline the similarities and differences that exist between industry and the health sector, as well as discuss the findings of research on the diffusion of medical innovations in the health care setting.

Although much of the diffusion evidence is conflicting there are a few issues for which consensus has been reached. For example, researchers agree that innovation involves multiple decisions and activities (Scott, 1991). This may be even more so among health care settings such as hospitals and public health units where innovation behaviour is likely governed by organizational processes. Scott (1990) also hypothesized that health care organizations were distinct in their organizational characteristics, and innovation decisions in such systems tended to be more decentralized and more localized to specialized units than in the typical organization. This statement is particularly relevant for public health units as they currently function. For example, program managers are directly responsible for making decisions about specific interventions and to some degree entire programs. Final approval however, comes first from the Medical Officer of Health, and ultimately the loca! board of health, which is comprised of local politicians from the city council. Therefore, individual decisions are made within the context of organizational processes and norms, environmental pressures, and social expectations.

Much of the diffusion evidence in the health care setting, as in all the other areas of diffusion research, is conflicting. As of the early 1990s only one characteristic, organizational 
size. was consistently found to be significantly associated with innovation adoption. Mixed results have been reported for characteristics such as centralization of decision-making, specialization and formalization. For example, some researchers found centralization of decisionmaking and vertical differentiation to be negatively associated with innovation adoption (Hage, 1973), while more recent work has found that centralization of decision-making is positively associated with innovation adoption (Shortell et al., 1989). The result of these conflicting findings is that there are still many unknowns related to the facilitators, barriers and process of innovation adoption. In addition, even less is known about these factors in the current public health setting.

\subsection{Barriers to Innovation in Organizations}

Considerable effort has been directed at understanding the barriers to innovation in health care settings and among health care professionals, which has resulted in a multitude of factors being identified. Among these are lack of time, lack of availability of relevant research findings, limited accessibility to research, limited literature retrieval and critical appraisal skills, negative attitudes toward research, lack of administrative support, and financial limitations (Heater et al., 1988). Scott (1990) also identified a number of organizational barriers which have been reduced into the four general categories of bounded rationality, vested interests, embeddedness, and institutionalization. The first three are most relevant to the public health sector and will be discussed further as they relate to public health structure and behaviour.

Scott (1990) refers to bounded rationality as the specialized objectives, differentiated roles and formalized structures that organizations use to effectively and efficiently achieve preestablished ends. Such systems are bounded in that these structures are only functional if the organization's goals remain constant and no changes in the environment occur. Other researchers 
have found that strict adherence to policies and procedures, rigid work routines and incentives stifle attempts to introduce change to bring about organizational innovation (Mirvis et al., 1977; Nelson et al., 1978). The results of this research suggested that specialization, formalization, and centralization are associated with an organization's innovative behaviour, but that this association can be positive or negative depending on the organization or the innovation.

Although none of these factors have previously been measured within public health units in Ontario. some information about these factors is known. First, the nature of the work public health units are mandated to provide indicates that health units are highly specialized in that they offer a large number of programs and services to a large variety of target audiences. Second, health units are highly structured with defined roles and work routines, as well as numerous policies and procedures to guide practice. Although less is known about centralization of decision-making, the current hierarchy within health units suggests that decisions are more centralized than decentralized. The current decision process for most health units is such that program managers report to program directors who report to the medical officer of health, who makes the final decision before presenting decisions to the local board of health and local politicians

Scott (1990) also recognized that professional public organizations, which include public health units, are impacted upon by their external environment, particularly other agencies and institutions which seek to build their concerns into the public organization's agenda. These other agencies are likely to believe that they have a legitimate right to impose their interests on the public organization. Scott (1990) refers to these types of factors as vested interests. Other research suggested that vested interests act as barriers when the organization employs more than one professional group. Nelson and Yates (1978) pointed out that different interests might 
emerge between professions thereby causing one profession to promote an innovation while another profession resists it. This category of factors is particularly important in public health because health units work in collaboration with many local agencies and hospitals to promote continuity of care across the spectrum of health care services, and are comprised of a number of health care professionals. Collaboration among institutions and agencies is necessary despite the competing interests and varying goals among these organizations. These varying interests may impact on the ability of public health units to make decisions to innovate. In addition, the recent downloading of public health services to the municipal level may have created even greater need for collaboration among the various health agencies within communities thereby having an even greater impact on innovative behaviours among public health decision-makers.

Scott's (1990) third category is embeddedness. Granovetter (1985) indicated that behaviour in organizations is embedded in concrete, on-going systems of social relationships. Granovetter demonstrated that interpersonal behavicur in organizations is constrained by informal relations and norms such as friendships. coilegial ties, norms of reciprocity, and trust. These ties connect persons and groups not only within organizations but also across organizations and can act as both facilitators and barriers to innovation. One such example in public health is the many networks that have been developed throughout the health regions on various health topics, which provide a venue for health professionals from different health units to work together to develop programs and services. This type of factor might promote innovation among health units, although it is possible that such networks could also function to inhibit innovation as well.

More recent innovation research suggested that systems whose members are closely linked by communication networks have a strong diffusion effect and a faster rate of innovation 
adoption (McKinney et al., 1991). In the public health field these communication networks might occur both within and external to the health unit. For example, there may be mechanisms in place that facilitate the flow of new ideas into the health unit from external sources. As well, members of the health unit might belong to external networks and work groups that promote awareness of innovative ideas, which members then bring back to their respective health units. Both alternatives were supported in the innovation literature which found that members of highly interconnected systems learned about innovation sooner and were more likely to be early adopters (Anderson et al., 1985; Becker, 1970a; Coleman et al., 1966; Kimberly, 1978).

The experience in health care is similar to that seen in the diffusion literature, with the exception being that most innovations take longer than fifteen years before being adopted (Lomas, 1991). The diffusion literature suggests that the time to adoption appears to be shorter when the inventor attempts to innovate as opposed to being content to merely reveal a generai concept (Utterback, 1974). A lesson to be learned from the diffusion literature is that greater emphasis should be placed by the researcher on the translation of research findings into easily implemented clinical practices. Recent examples of such strategies include the Evidence-Based Medicine, Nursing and Mental Health journals which provide commentaries for each research study about how the evidence can be used in practice.

\subsection{Characteristics of the Innovation, Organization, Environment and Individuals}

Findings from the diffusion of innovation literature as well as the research dissemination and utilization literature were used to identify and define the independent variables examined in this dissertation. The following section will describe in detail all the characteristics included among the four main elements of innovation characteristics, organizational characteristics, environmental characteristics and finally individual characteristics. 


\section{Characteristics of the Innovations}

A number of characteristics of the innovation found to be associated with the diffusion of innovations have been identified in the literature (Battista, 1989; Lewis et al., 1993; Poole et al., 1990; Rogers, 1995). Poole and DeSanctis (1990) suggested that at both the individual and organizational level, user's level of comfort in using the innovation and level of respect for the innovation will determine user's adoption behaviour. Rogers (1995), has identified five specific attributes that potential users utilize to assess the advantages and disadvantages of innovations. These include the relative advantage of the innovation, compatibility, complexity, trialability and observability. Although five attributes have been identified, three, relative advantage, compatibility, and complexity, were measured during the OHCEN follow-up survey in 1996. The focus group discussions conducted in 1996 with public health decision-makers, identified these three attributes as the most applicable and relevant with respect to utilization of the systematic overviews, and therefore data on trialability and observability were not collect at that time. This method is supported by Rogers (1995) who indicated that the main attributes of an innovation should be elicited from the respondents prior to measuring them. In addition, issues related to recall bias, since the follow-up data collected for this dissertation were collected two years after the systematic overviews were disseminated, prevented additional data on trialability and observability from being collected in the 1998 survey. The three attributes included in this study have been further defined and operationalized below using the diffusion of innovation literature as well as the research utilization literature.

Relative advantage of the innovation has been defined as the degree to which an innovation is perceived as better than the idea it supersedes, and can be measured in economic terms. social prestige, convenience and satisfaction (Davis et al., 1997; Rogers, I995). In fact, a 
review of 23 randomized controlled trials measuring the effectiveness of guideline dissemination found that guidelines that were perceived as relatively uncomplicated were adopted to a greater extent than those perceived as complicated (Grilli et al., 1994). Rogers (1995) also defined subdimensions of relative advantage as a decrease in discomfort, social prestige and savings in time and effort. These definitions as well as feedback obtained in the focus group discussion in 1996. led to the development of several measures of the relative advantage of the systematic overviews on public health decision-making. The two subdimensions most applicable in this study were social prestige and savings in time and effort. A measure of social prestige included the priority that respondents believed should be given to conducting systematic overviews. Measures of savings in time and effort were measured by asking respondents their perceptions of whether systematic overviews could overcome barriers such as limited access to literature, not having enough time to review the literature, limited critical appraisal skills. limited resources, lack of relevant research to decision being made, unsupportive work environments and lack of decision-making authority.

Compatibility refers to the degree to which an innovation is perceived as being consistent with the existing values, past experiences, and needs of potential adopters (Rogers, 1995). Therefore potential adopters who perceive the innovation as being consistent with their values and experiences or their organization's values are more likely to adopt the innovation than those who do not. Rogers (1995) also indicates that previous practice provides a familiar standard against which an innovation can be interpreted, which may decrease uncertainty. One way to measure existing values would be to ask respondents about their perceptions of the weight that is routinely given to research evidence in decision-making at their health unit, as well as the weight they perceive should be given to research evidence in decision-making. Needs of the potential 
adopters could be measured by asking respondents their perceptions of the relevance of the systematic overviews to decisions being made as well as the appropriateness for receiving the systematic overviews.

Complexity represents the degree to which an innovation is perceived as difficult to understand and use (Rogers, 1995). A survey of midwives in 1993 in the UK demonstrated that these health professionals preferred to read research studies that were written in plain English, had minimal statistical data that was explained in uncomplicated language, were presented in an attractive way using high quality print and illustrations as much as possible (Meah et al.. 1996). A number of research utilization studies also supported the finding that complexity of research evidence was positively associated with research utilization (Hunt, 1981; Rodgers, 1994), meaning the easier the research was to use, the greater its utilization. Therefore in this study complexity was measured by asking respondents their perceptions of how easy the overviews were to use.

There is still more diffusion literature that discusses the impact of 'bandwagon pressures' on adoption behaviour. The literature suggested that organizations imitate other organizations that are proximate either geographically or in their communication networks, and therefore will adopt innovations when these other organizations have adopted an innovation (Abrahamson, 1991; Abrahamson et al., 1993; Brown, 1981; Burt, 1987). Therefore measures of bandwagon effects are important characteristics of the innovation that should be included in this diffusion study. Abrahamson \& Rosenkopf (1993), also indicated that bandwagons are diffusion processes whereby organizations adopt an innovation, not because of their individual assessments of the innovation's efficiency, but because of a bandwagon pressure caused by the sheer number of organizations that have already adopted the innovation. This type of bandwagon pressure is 
likely to exist among public health units, particularly when competition for limited resources exists. However, since decisions concerning programs and services are both an individual and organizational process, bandwagon pressures could be perceived at either level. Organizational theorists claimed that the number of organizations that adopt an innovation in the early stages of the diffusion process create pressure, causing other organizations to adopt in the later stages (Utterback, 1974). These claims are useful in understanding why some innovations in health care are widely accepted before their effectiveness is really known, and why others that are proven effective continue to be underutilized.

Two measures of bandwagon effects emerged from this literature. The first involved public health decision-makers' perceptions of the extent to which public health units across Ontario were using the systematic overviews for decision-making. The second measure occurred at the individual level, and measured public health decision-makers' perceptions of the extent to which colleagues in the same positions as themselves were using the systematic overviews for decision-making. Therefore the final two innovation characteristics will seek to determine if a bandwagon effect played a part in the adoption/ utilization of the systematic overviews among public health decision-makers. Neither measure has previously been assessed among public health decision-makers in Ontario.

\section{Organizational Characteristics}

The diffusion research also demonstrated the importance of organizational factors with respect to adoption behaviour, with a number of research findings indicating that the organizational context has had a major influence on health professional's and organizations' innovation behaviours (Battista, 1989; Kaluzny et al., 1974; Kitson et al., 1996). In fact, Scott (1990) suggested that organizational characteristics were the most important factors associated 
with innovation adoption. An early study conducted on a national sample of American hospitals demonstrated that organizational variables accounted for $41.0 \%$ of the observed variation in innovation among health care professionals, while individual characteristics of the chief administrator of the hospital accounted for only 5 percent of the observed variation (Veney et al., 1971). Organizational characteristics may be even more important among public health decisionmakers since these health professionals, more so than physicians practicing within solo or small group settings, must work within the policies and procedures set out by provincial and local governments.

A large number of organizational characteristics have been examined during the last three decades of diffusion of innovation research. The most commonly studied organizational variables included: organizational complexity, centralization of decision-making, formalization of rules and behaviour, size and resources (Greer, 1977; Lewis et al.. 1993). Damanpour (1991) defined a number of organizational characteristics in conducting a meta-analysis of the diffusion of innovation among health care professionals, seven of which were included in this dissertation. Organizational complexity was defined as the number of distinct services an organization provides. In public health this characteristic would represent the number of services and programs the health units provide. Centralization was defined as the extent to which decisionmaking authority was dispersed or concentrated in an organization. He defined formalization as the extent to which an organization followed rules and procedures in conducting organizational activities, which in public health could be measured by obtaining decision-makers' perceptions of the degree to which policies and procedures were adhered to in their health unit. Functional differentiation was defined as the extent to which an organization was divided into different divisions. In public health this characteristic would be represented by the number of divisions 
within the health unit, such as Nursing, Healthy Lifestyles and Communicable Diseases. Financial (slack) resources were defined as resources an organization has beyond what it minimally requires to maintain operation, which in public health could be measured by obtaining decision-makers' perceptions of whether or not adequate financial resources were available in the organization. External communication was defined as an organization's ability to be in contact with and scan its environment. This variable can be measured in public health by obtaining decision-makers' perceptions of whether mechanisms exist in their health unit that facilitate the transfer of new information from the environment into the organization. The final characteristic used from Damanpour (1991), was vertical differentiation which was defined as the number of levels in an organization's hierarchy, which in public health would be the number of levels of workers from front line staff up to the medical officer of health.

Organizational characteristics found to be significantly associated with the adoption of innovations included: organizational size, complexity, available resources, functional differentiation, administrative intensity (proportion of managers to staff), culture, internal and external communication channels, and decision-making processes (Lewis et al., 1993; Greer, 1977: Kaluzny, 1974; Kimberly, 1978; Kimberly et al., 1981; Scott, 1990; Utterback, 1974; Becker, 1970b: Kaluzny, 1974; McKinney et al., 1991; Hunt, 1996; Lomas, 1993b).

Damanpour's meta-analysis in 1991 included 23 published empirical studies that measured the rate of adoption of innovations among individuals. The results of this meta-analysis demonstrated a positive association between staff member innovation and functional differentiation, slack resources and extemal communication networks, and nonsignificant associations between innovation and formalization and vertical differentiation. Therefore, these findings suggested that organizations with more divisions or departments, with excess financial 
resources and mechanisms that allowed the flow of external information into the organization had higher rates of innovation adoption among decision-makers.

However, the nonsignificant association for formalization was surprising to the researchers given their hypothesis that adherence to strict policies and procedures would be significantly associated with lower rates of adoption. They hypothesized in their discussion that it was likely that some degree of formalization might be necessary to facilitate innovation adoption. Therefore, the evidence was inclusive for formalization, and further examination of this variable is warranted in other fields. The results also demonstrated that vertical differentiation was not significantly associated with innovation adoption when all types of innovations were examined. However, they did find a positive significant association when the innovation was an administrative innovation. Since the innovation in this study was an administrative innovation, the literature suggested that vertical differentiation be included as an independent variable, and that it (vertical differentiation) would be associated with research utilization.

Findings from the diffusion literature also demonstrated that the more diverse an organization was in terms of the variety of services it provided (complexity), the greater the likelihood of innovation adoption among decision-makers (Greer, 1977; Hage et al., 1973; Kaluzny et al., 1974; Kimberly, 1978). In fact Kaluzny (1974a) reported that the complexity of the organization and organizational value accounted for $60 \%$ of the observed variation in organizational program innovation, among a random sample of hospitals in the United States.

The effect of centralization of decision-making was less clear in this literature as some findings suggested that organizations that were decentralized with respect to decision-making had greater rates of innovation adoption among staff members. (Greer, 1977; Hage et al., 1967; 
Kaluzny, 1974; Rodgers, 1994). However, more recent studies have brought into question the positive association between decentralization and individual innovation (Hage et al., 1973). Although there exists in the literature some ambiguity as to the impact of the organizational decision-making process on innovation behaviour, there was enough evidence supporting some type of relationship between these two factors to warrant further examination in this study.

Lomas, (1997) suggested that whatever potential there is for greater use of evidence in decision-making, it cannot be realized until significant improvements are achieved in the linkages between current or future research decisions on pressing or emerging issues. Decisionmaking may have a significant impact on the process of transferring research evidence into practice, and therefore should be included in models of research dissemination and utilization. Even though concepts of decision-making theory are well entrenched in the management literature. and in fact have been studied extensively with regard to organizational decisionmaking, they have received much less attention from dissemination researchers in the health care field.

Recent work in Saskatchewan has indicated that decisions are made based on the values of those making the decision, individual experiences, stakeholder interests, and 'evidence' (Kouri, 1997). However, little is known about how each factor interacts with the other, or how the decision-making process in general interacts with individual, organizational, environmental and innovation characteristics. What is known is that early and ongoing involvement of relevant decision-makers in the conceptualization and conduct of a study is the best predictor of its utilization (Lomas, 1997). This evidence supports ongoing efforts to examine the relationships between research dissemination and utilization, and individual and organizational decisionmaking processes. 
Advocates of research dissemination have questioned why such long gaps exist between the introduction of new knowledge and changes in clinical practice. It has been argued that in cases where this delay occurred, the political and institutional context was initially misaligned with the findings. Decisions based on these insights therefore did not flow into useable knowledge until the research resonated with other contextual factors, providing a justification for its use or corroborating its value (Lomas, 1997). These findings confirm that decision-making is a complex issue comprised of many factors that are not well understood, but which are related to research utilization. As was the case with many of the organizational characteristics, decisionmaking is a concept in need of further study to clarify its definition and measurement and to determine its impact on research utilization.

Formalization was generally believed to have a negative effect on innovation adoption as this process inhibits the flow of communication among members of the organization. which reduces awareness of new ideas (Greer, 1977; Kaluzny, 1974). Therefore, adherence to policies and procedures would be expected to be associated with less research utilization in this study.

Although the organizational theory literature concurs with many of the organizational characteristics identified in the innovation literature, it also has identified and defined the important characteristic of organizational culture. The impact of culture has been examined in a number of diffusion of innovation studies as well as organizational change processes, but has only recently begun to be considered an important factor in research dissemination and utilization studies. Culture has been defined as the pattern of basic assumptions and shared meanings (values) that a group develops to survive their tasks; works well enough to be taught to newcomers; and is a pattern of behaviours that is unique to each group (Mark, 1996; McSkimming, 1996; Van Ess Coeling et al., 1993a; Closs et al., 1994; Hofstede, 1980; Reichers 
et al., 1990; Shortell et al., 1991). Cultural behaviours fall into four categories: the work group's priorities. power issues, peer relationships, (extent to which group members work together or alone), and group preferences for permanence or change (Van Ess Coeling et al., 1993a; Van Ess Coeling et al., 1993b). Since many or all of these behaviours may be involved in the implementation of research evidence into practice, it seems reasonable to expect that organizational culture may be associated with the success or failure of dissemination strategies to produce innovation adoption/research utilization. In this study culture was defined as the pattern of basic assumptions and shared meanings (values) that a group develops to survive their tasks.

Organizational culture has been measured as both an independent and dependent variable. As an independent variable, the impact of culture on organizational outcomes is sought, whereas as a dependent variable, culture is used to explain why members of the organization behave as they do (Thomas et al., 1990). Both of these approaches are applicable to understanding innovation adoption and research utilization in health care organizations and in particular public health units. One research study suggested that understanding a nursing unit's culture might assist in the process of introducing a new practice model (Coeling et al, 1993). Therefore, it seemed reasonable that understanding culture in public health units might also assist in facilitating the utilization of systematic overviews for decision-making. In this study organizational culture was used as an independent variable and assessed whether it was associated with utilization of systematic overviews.

The literature also demonstrated that the value the organization and individuals within the organizations placed on research utilization was strongly associated with research use (Funk et al.. 199lb; Kaluzny et al., 1974). For example, Kaluzny (1974a) found that the values of senior decision-makers were slightly more strongly predictive of organizational innovation than were 
organizational structure or resource variables. This is particularly important in the public health setting because it suggests that public health units which valued the use of research evidence in decision-making will likely demonstrate greater use of the systematic overviews in decisionmaking. Another study whose sample was comprised of directors of nursing from community hospitals in Ontario, found directors were more likely to utilize research evidence in decisionmaking if research committees with nursing representation and researchers had been established (Royle et al., 1997). A link can also be made between the development of these committees and the value the organization places on research evidence. Therefore, there is fairly conclusive evidence that valuing research is a strong predictor of research utilization.

There is considerable variation across organizations regarding culture type. and some cultures are strong in constructive behaviours which lead to increased teamwork and goal setting (Thomas et al., 1990). This suggests that different culture types may impact on an organization's ability to incorporate research evidence into daily practice and decision-making. The organizational theory literature indicates that in order for strategies to be effective, they must address the language, assumptions, symbols, and values of the current culture or the desired future culture (McSkimming, 1996; Cameron et al., 1991; Denison et al.. 1991; Pettigrew, 1979). This concept is congruent with other work that suggests that dissemination strategies must be tailored to the needs of the target audience if they are to be effective (Dobbins et al., 1998).

Organizational culture is important not only as a vehicle for implementing change (Cameron et al., 1991; Deal et al., 1982; French et al., 1984), but also as a targeted outcome in systematic change efforts. The underlying assumption is that a better understanding of organizational culture can be used to improve organizational performance (Mark, 1996). In terms of dissemination research and utilization, the assumption is that culture can be used to improve 
organizational performance by encouraging the transfer and uptake of research evidence into daily practice and decision-making. However, there is room for considerable growth in this area, especially in Canada, where the study of culture in health care organizations has been minimal.

Organizational culture represents one of many factors associated with innovation and research utilization among public health decision-makers, and was assessed in this exploratory study by measuring decision-maker's perceptions of the value their organizations place on using research evidence for decision-making. and by the extent to which decision-makers perceived unsupportive work environments were a barrier to using research evidence.

Lewis (1993) indicated that examination of adoption processes must also incorporate organizational characteristics such as communication patterns. This is further supported by the results of a number of studies demonstrating that communication patterns within organizations that facilitate the flow of information into and within an organization are associated with innovation (Hage, 1974; Kimberly, 1978; Mott, 1972; Rogers et al., 1971; Zaltman et al., 1973). Therefore, there is consistent evidence that communication patterns that facilitate the flow of new information into the organization is associated with innovation. In public health units this can be operationalized as the presence of mechanisms that facilitate the transfer of new information into the health unit.

The final type of organizational characteristic examined in the literature has been labeled organizational context variables. These include measures of size and available resources. A number of studies have demonstrated organizational size as an important predictor of innovation (Kaluzny et al., 1974; Lewis et al., 1993). Other studies have suggested that the location of the organization, such as urban or rural was a major influencing factor (Rodgers, 1994). In addition 
available (slack) resources have been shown to be positively associated with organizational innovation (Milio, 1971; Mulhall, 1992).

\section{Environmental Characteristics}

Both the diffusion and research utilization literature over the last two decades have started to discuss the importance of environmental factors in relation to the diffusion of innovations and the utilization of research evidence. In fact, Abrahamson (1991) suggested that environmental changes could engender new performance gaps among organizations with similar goal orientations, prompting them to adopt innovations. Environmental changes, as demonstrated by the downloading of public health services to the municipalities in 1998, represent a constant and key factor currently influencing all public health units in Ontario. As public health units seek to justify resource requirements and services, there is the potential for the adoption of administrative innovations to facilitate justification efforts. Early diffusion work also suggested that innovation was a function of its environment, which included, economic, social and political factors (Baidridge et al., 1975; Kaluzny, 1987; Kotter, 1979; Scoth, 1993; Utterback, 1974).

Lewis (1993) demonstrated that environmental forces could significantly impact on an individual's and/or organization's innovation adoption behaviour. Environmental factors related to the socioeconomic infrastructure of the community have been shown to impact on such organizational structures as administrative intensity, formaiization, and centralization of decision-making (Rogers, 1988). The factors most applicable to the public health units included in this study were geographic location (urban versus rural), and the existence of collaboration and networks among community agencies. Other environmental factors that have been shown to be associated with innovation adoption include reporting relationships between the top management team and the board, regulations and legislation, urbanization, network 
embeddedness, financial resources, peer pressure, competition between institutions to attract specialized professionals, and acquisition of prestige in the community (Battista, 1989; Burns et al.. 1997; Cockerill et al., 1996; Kimberly et al., 1981; Meyer et al., 1988).

Warner (1975) pointed out however, that little attention has been paid to the impact of environmental factors on innovation diffusion, despite its importance in medical decisionmaking. This was also evident in the public health literature, as little to no emphasis has been placed on examining the impact of environmental characteristics on public health decisionmaking. Kaluzny (1974b) pointed out the importance of local politics and political influence on innovation behaviour in the public health setting, by suggesting that the extent to which the actions of the local board of health are independent of local community values is positively associated with program innovation. This finding is particularly important in the current public health context since some members of the local board of health are locally elected politicians on the city council. Therefore, to some extent these board members are governed by local community values. However, little is known at this time whether Kaluzny's (1974b) findings remain true. It is important at this time to deternine if an association exists between the actions of the local board of health and the innovat:on behaviours of the local public health decisionmakers. The environmental characteristics inclucied in this study were the perception that nules and regulations of provincial and local governments influence decision-making, the perception that health unit decisions were made in ccilaboration with local agencies, perceptions of the influence the medical officer of health had on the local board of health and local politicians and perception of the extent to which local board members and politicians are influenced by research evidence. 


\section{Individual Characteristics}

Even though research supported the concept that innovation within organizations was dependent upon a number of organizational factors, medical care organizations are uniquely different from other organizations and therefore the characteristics of the individual cannot be ruled out as having a significant impact on innovation behaviour. For example, medical organizations are believed to contain numerous types of professionals, which is consistent with current employment patterns in public health units in Ontario. These types of professionals have been found to experience considerably more autonomy in decision-making than staff in typical organizations, and therefore for some innovations, the relevant decisions and adoption unit may be an individual professional practitioner rather than an organization (Scott, 1990). In addition, the organizational theory literature illustrated that autonomy was positively related to productivity, and specifically that autonomy was associated with decision-makers acquiring more relevant work knowledge (Pearson, 1992). This may be a likely event in public health units, as program managers experience substantial autonomy in decision-making concerning program development and the provision of specific interventions. Therefore, when assessing innovation behaviour both organizational and individual characteristics must be considered.

The research utilization literature, diffusion of innovation literature and organizational theory literature have identified a number of individual characteristics that facilitate research utilization, as well as a number of characteristics that act as barriers to utilization. Rogers (1995) also indicated that most individuals do not evaluate an innovation on the basis of scientific studies of its consequences, but instead depend mainly on a subjective evaluation of an innovation that is conveyed to them from other individuals like themselves who have previously 
adopted the innovation. This finding suggests that the degree to which individuals interact with others who have adopted an innovation might be influenced by this interaction.

The literature identified characteristics such as cosmopolitanism (the number of days spent at conferences, workshops, or interacting with others outside of current organization), and network embeddedness (collaboration with other organizations), as potentially important factors in the research utilization process. This statement has been supported by a number of research utilization studies (MacGuire. 1990; Titler et al., 1994), which demonstrated that research utilization was associated with attendance at conferences and reading research journals (Coyle et al.. 1990; Michel et al., 1995). Other research has found that research utilization is positively linked with being involved in research, and less associated with attitudes toward research (Bostrum et al., 1993; Kimberly, 1978), and that access to a research consultant with whom the intended audience has confidence in is also positively associated with research utilization (Strang et al.. 1995).

The diffusion literature demonstrates that individual characteristics such as position, seniority, age and authority are important factors in the innovation adoption process (Lewis et al.. 1993), as well as(Kaluzny, 1974) education, type of specialization, tenure and participation in ongoing training (Battista, 1989; Kaluzny, 1974; Kimberly et al., 1981: Lewis et al., 1993; Rogers, 1983). Rogers has identified prior conditions such as previous practice, felt needs, and innovativeness, as important factors to consider in the knowledge stage of the diffusion process (Rogers, 1983). However, in some studies positive relationships were observed while in others negative relationships occurred. Therefore, it is unclear which direction should be expected for a number of these characteristics in this dissertation. 
Kaluzny, (1974) identified a number of barriers to utilizing research evidence among individual decision-makers. For example, the literature demonstrates that many health care professionals do not perceive research findings to be relevant to their practice and therefore are not prone to consider using this evidence in decision-making (Bero et al., 1997; Funk et al., 1991a: Funk et al., 1995; Greenwood, 1984; Miller et al., 1978; Titler et al., 1994; Walczak et al. 1994). As well, the majority of health care professions work within multidisciplinary teams, within complex organizations with a multitude of rules, regulations, policies and procedures. Health care professionals in these positions may not have the authority to implement practice changes, particularly if these changes require financial resources or resource allocation. This was supported by a large random survey of 5000 nurses in 1990, who were asked to identify the three greatest barriers to research utilization. The greatest barrier was limited decision-making authority to change patient care procedures, while the second greatest barrier was insufficient time to implement new ideas and review literature (Funk et al., 1991b; Funk et al., 1995; Hunt, 198 1: Lacey, 1994; Pettengill et al., 1994; Stolk et al., 1995; Walczak et al., i994).

The third most important barrier was perceived lack of administrative support for implementing evidence-based practice (Hicks, 1996; MacPhail, 1997; McSherry, 1997; Pettengill et al., 1994). Perceived availability of research evidence has also been found to be a significant barrier to research utilization (Champion et al., 1989; Cronenwett, 1995; Hunt, 1981; Luker et al., 1992). Another important barrier identified in a number of research utilization studies was limited critical appraisal skills. Many health care professionals have reported a limited ability to understand research findings and to critique the research methods of the study (Funk et al., 1995; Hicks, 1996; Hunt, 1981; Luker et al., 1992; Marshall, 1993; Stolk et al., 1995; Veeramah, 1995; Walczak et al., 1994; White et al., 1995). Carter and Williams (1957) 
noted that if a firm was to make rapid and effective use of scientific ideas, it must include people capable of evaluating or interpreting these ideas in accordance with the needs of the firm. Although this finding was demonstrated several decades ago, it was an early indication of the important role critical appraisal skills played in the diffusion of innovation. Current research dissemination and utilization research has now corroborated the importance of critical appraisal skills in not only in the diffusion of innovations but also research utilization. Availability of research evidence has also been identified in several research utilization studies as having a significant impact on whether research evidence was used in practice (Stetler, 1994; Veeramah, 1995), as well as the extent to which decision-makers routinely read research literature. A random sample of nurses in Canada demonstrated that one third of the sample reported not reading research literature on a regular basis (Funk et al., 1995; MacPhail, 1997). Therefore, there are a number of individual characteristics that have been identified in the literature that promoted the use of research evidence, and an equally large number of characteristics that are perceived as barriers to using research evidence in decision-making.

\subsection{Dependent Variables: Innovation Adoption/Research Utilization}

Rogers (1995) identified three types of innovation decisions that can occur in a social structure. These include optional innovation decisions, collective innovation decisions, and authority innovation decisions. Although all three were relevant to innovation decisions in public health. only the first one fits the focus in this dissertation. Optional innovation decisions are defined as choices to adopt or reject an innovation that are made by an individual independent of the decisions of the other members of the system (Rogers, 1995). This means that the unit of analysis is at the level of the individual decision-maker. This is the most applicable unit of analysis for the participants in this study, since the adoption behaviour of individual decision- 
makers such as managers, directors and associate/medical officers of health were examined. Although collective innovation decisions, which are organizational choices to adopt or reject an innovation made by consensus among the members of a system, might also be applicable in this study. the small sample of public health units available for this study, precludes that level of analysis at this time.

Methodological advancement in the study of the diffusion of innovations has impacted on the conceptualization of the outcomes of the innovation adoption process. Whereas the outcome of the diffusion process has traditionally been measured in a binary form (adopt/not adopt), more recently researchers have determined that adoption is not an all or nothing process (Calsyn et al., 1977; Larsen et al., 1977). Research has demonstrated that similar organizations will adopt innovations in very different ways, and that dissimilar organizations will adopt innovations in similar ways. As well, the diffusion literature points out that technologies that are available for a specific indication will not only be used at significantly different intensities in various countries and regions, but will also be used for different purposes among adopters (Gelijins et al., 1994). The variation in adoption observed in these studies led to the realization that there were many other ways in which to measure innovation aside from the traditional adoption versus no adoption, and that innovation could and should be measured along a continuum from no adoption to full adoption. Since the decisions made by public health decision-makers concerning service provision were partially determined by the needs of the local community, it seemed reasonable to expect that the systematic overviews would be adopted in varying ways across the province. Therefore, several options for measuring research utilization were available. However, since measurement of adoption of this type of innovation had not previously occurred in public health, the results of the original focus group discussions in 1996 were used to guide the development of 
outcome measures. The focus group discussions resulted in six outcomes specific to public health decisions being identified. These included an overall measure of the use of the systematic overviews, and the extent to which the overviews influenced what were considered to be key decisions in public health. These were program planning, program justification, program evaluation, policy development and staff development. 


\subsection{Research Methods}

A cross-sectional observational study design which included a telephone survey of decision-makers from all public health units in Ontario, and a self-administered organizational demographic questionnaire completed by one administrative assistant in each health unit, was used to assess the utilization of five systematic overviews, two years after their dissemination. Respondents were asked to provide information on their perceptions of their organization and their environment. Data on individual characteristics and the decision-makers' perceptions of characteristics of the systematic overviews were collected during the OHCEN study in 1996. Data from those decision-makers who participated in the OHCEN study were then merged with the data collected from these same decision-makers in the current study.

Use of the five systematic overviews since their dissemination in 1996, (the dependent variable) was measured in two ways. The first was as a dichotomous variable of use versus no use. while the second measure of use examined the extent to which the systematic overviews influenced decisions related to program planning, program justification, program evaluation, policy development and staff development. The latter were measured using a five point likert scale and were analyzed as continuous variables throughout the analysis, as has been suggested in recent work on analyzing categorical data in public health research (Preisser et al., 1997).

\subsection{Setting and Sample}

The study sample included all decision-makers employed in public health units in Ontario who had completed both surveys in the OHCEN study in 1996. The study sample included medical officers of health, associate medical officers of health, program directors and program managers who were responsible for making program decisions about public health practice. The 
sample was designed to include all public health units in Ontario. The unit of analysis was individual public health decision-makers.

The medical officer of health in each public health unit was sent an introductory letter explaining the study and asking for the participation of their public health unit. The primary investigator then called each medical officer of health within two weeks to answer any questions concerning the study and obtain consent to participate in the study. Once consent was obtained from the medical officer of health, public health staff who paricipated in the OHCEN study in 1996 were contacted initially by letter and then followed up by phone to obtain their consent to participate in the study. Upon receiving this consent a time was scheduled for a fifteen minute telephone interview. A short mailed, self-administered questionnaire was also sent to each medical officer of health's administrative assistant. Questions included in this self-administered questionnaire were related to organizational demographic information such as the size of the health unit, number of programs and services offered and involvement in research studies.

\subsection{Measures}

The independent variables of interest included characteristics of the organization, environment, innovation and individual. A full list of the independent variables with operational definitions and methods for measurement is summarized in Table 1 , and a complete list of the survey questions is presented in Appendices 1-4.

\section{Independent Variables}

Organizational Variables

Appendices 1 and 2 display the specific survey questions used to collect data on the organizational characteristics. To facilitate easier identification of the specific survey questions, the number of the question will be provided in brackets following a description of the variable. 
Data collected on the following eight organizational characteristics were collected using the questions from Appendix 2. These included 1) the number of full time equivalent personnel (11315);2) urban/rural mix of population served $(11317) ; 3)$ number of divisions $(11318) ; 4)$ number of programs (11319); 5) number of levels of workers $(11320) ; 6)$ involvement in research activities (11322);7) number of ongoing research projects in health unit (11321); and 8) teaching health unit status (11324). Data collected on the remaining seven variables were collected using questions from Appendix 1. These included 1) formalization of rules (21772);2) perceived adequate resources to implement decisions based on research evidence $(21774)$; 3 ) perceived value the organization places on research evidence $(21773)$; 4) perceived ongoing training in research methods and critical appraisal to staff $(21775) ; 5)$ perceived existence of integrative mechanisms (mechanisms to allow external information to flow into the organization) $(21776) ; 6)$ perceived extent to which information is routinely searched for before making decisions $(21783) ; .7)$ and perceived importance organization routinely places on research evidence to make decisions (21786).

\section{Environmental Variables}

Data on the following six environmental characteristics were collected using questions from Appendix 1. These include: 1) perception that provincial and municipal regulations and legislation influence decision-making $(21790) ; 2)$ perceived influence the medical officer of health has on the local board of health $(21792) ; 3)$ perceived influence the medical officer has on the local politicians $(21793)$; 4) perception that decisions are made in collaboration with local agencies $(21791), 5)$ perception of the extent to which research evidence influences the local 
board of health (21794); and 6) perception of the extent to which research evidence influences local politicians (21795)

Characteristics of the Innovation

Data on all but two of the 23 innovation characteristics were collected during the OHCEN study in 1996. Respondents' perceptions of characteristics of the systematic overviews. such as relative advantage, compatibility, and complexity were measured during the baseline survey in May/June of 1996 before receiving the systematic overviews. Most of these characteristics were then measured a second time in the follow-up survey in August/Sept of 1996. The data that were collected during the baseline OHCEN telephone survey are denoted as occurring in Time 1 (TI) and the data collected in the follow-up OHCEN survey are denoted as occurring in Time 2 (T2). A number of Likert scales were used to measure the respondents' perceptions of characteristics of the overviews, as summarized in Table 1, and Appendices 3 and 4. For example, to assess respondents' perceptions of the relative advantage of systematic overviews, respondents were asked to rate the priority that should be given to conducting systematic overviews on a four point Likert scale ranging from "low priority" to "top priority". This variable was measured at both T1, before respondents received the overviews and at T2, three months after receiving them. All of the Likert scales used to measure perceptions of the innovation characteristics were either four or five point scales. Data on the two remaining characteristics were collected in 1998 and focused on respondents' perceptions of a bangwagon effect. These two innovation characteristics were measured using a continuous scale from $0 \%$ to $100 \%$ 
Respondents' perceptions of the relative advantage of the systematic overviews were assessed using a series of questions. The first asked respondents' their perceptions of the priority that should be given to conducting systematic overviews. This was measured at T1 and T2. Respondents were then asked their perceptions concerning systematic overviews being able to overcome barriers to using research evidence for program decision-making. Using a five point Likert scale, ranging from "definitely won't" to "definitely will", respondents were asked if they perceived systematic overviews would overcome the following barriers: 1) limited access to literature, 2) not enough time to review literature, 3) limited critical appraisal skills. 4) limited resources, 5) lack of research relevance to program decisions, 6) work environments that were unsupportive of using research evidence, and 7) lack of decision-making authority. Data on all of these characteristics were collected at both $\mathrm{T} 1$ and $\mathrm{T} 2$.

Respondents' perceptions of the compatibility of the systematic overviews were measured by 1) asking their perceptions of how much weight was given to research evidence in their health unit when program decisions were made, and 2) how much weight they personally believed should be given to research evidence when making program decisions, 3) whether the systematic overview was relevant to decisions that were being made and 4) the appropriateness of the timing for receiving the overviews. Data on the first two measures were collected at $\mathrm{Tl}$ using a five point Likert scale, while the latter two were collected at T2 using a four point Likert scale.

Respondents' perception of the complexity of the systematic overview was measured using one basic concept: were the systematic overviews easy to use. Respondents' were asked to comment on this measure using a four point Likert scale ranging from "poor" to "excellent", and was measured at T2. 
The final two innovation characteristics measured whether a bandwagon effect was influencing the adoption of the systematic overviews. Data on both of these independent variables were collected in 1998 and assessed respondents' perceptions of the extent to which other health units and colleagues across Ontario were using the systematic overviews for decision-making. The first measure asked respondents about their perception of the percentage of health units in Ontario that were using the systematic overviews to make decisions, and the second asked their perceptions of the percentage of colleagues, in the same position as themselves. who were using the overviews to make decisions.

Individual Characteristics

Data for fourteen characteristics of the individual were also included in this analysis. The data on individual characteristics were collected during the baseline OHCEN study in 1996. However, respondents were asked in the current study in 1998 to clarify that the demographic data collected in 1996 were correct. The survey questions used to assess these individual characteristics are presented in Appendix 3. The individual characteristics included 1) current position in the health unit (typ); 2) years in current position (21766); 3) years in public health $(21767) ; 4)$ years since graduation $(21769) ; 5)$ age $(20793) ; 6)$ access to a consultant to help with interpreting research (20705); 7) number of articles retrieved in past month (20707); 8) number of retrieved articles read in the past month (20708); 9) number of days spent at conferences in the past year $(20715)$; 10) direct access to on-line database searching $(20709)$; 11) use of systematic overviews in the past $(20745) ; 12)$ the perception of the degree to which lack of decision-making authority was a barrier to using research evidence (20726), 13) the perception of the degree to which limited critical appraisal skills were a barrier to using research evidence 
(20722); and 14) the perception of the degree to which limited resources were a barrier to using research evidence (20727).

\section{Dependent Variables}

There were six dependent variables measured in this study. The first was an overall measure of use of the systematic overviews, while the remaining five measured the influence of the systematic overviews on five key decision-making areas in public health. Overall use of the systematic overviews was measured as a dichotomous variable. To measure overall use respondents were asked if they had used any of the five systematic overviews during the past two years to make decisions concerning public health practice. The remaining five dependent variables were measured using a five point Likert scale ranging from "Not at all" to "A Great Deal". Respondents were also asked to comment on the extent to which they perceived the systematic overviews had influenced decisions related to the five key public health decisions of 1) program planning. 2) program justification, 3) program evaluation, 4) policy development and 5) staff development. Although the influence the systematic overviews might have on these five public health decisions may be correlated, they were examined as separate variables in this study due to the importance placed on them by public health decision-makers in focus group discussions during the OHCEN study in 1996. The focus group discussions illustrated the potential ways in which public health decision-makers perceived systematic overviews could impact on public health practice. Since this was the first attempt at measuring these utilization outcomes in public health it was important to examine each of the outcomes separately to identify if they were appropriate outcome measures. Future research efforts might examine scaling all of these outcomes into one measure of influence. However, at this point, more data on 
each potential outcome are necessary in order to facilitate ongoing research and the development of future testable hypotheses and dissemination strategies in this field.

\subsection{Instruments}

The data for this dissertation were collected using four instruments. Two of the instruments, presented in Appendices 1 and 2, were developed for this study. Appendix I presents the questions used to collect data on the organizational, environmental and the two bandwagon characteristics, as well as the six research utilization outcomes. Appendix 2 presents the survey questions used to collect the organizational demographic data. Both instruments were pre-tested for test-retest reliability and face validity at one public health unit. A total of twelve decision-makers participated in testing the reliability of Appendix 1. The questionnaire was administered by telephone and lasted approximately 20-25 minutes. Following completion of the questionnaire, respondents commented on the wording of the survey questions and made recommendations for changes. The overall Chronbach alpha score for Appendix 1, based on the responses of these twelve decision-makers, was 0.65 , which was considered adequate for this study. In addition, five of the twelve decision-makers agreed to participate in a test-re-test of the questionnaire. This involved completing the questionnaire by phone twice, one week apart. The intraclass correlation coefficient was used to identify specific items in the questionnaire that produced unreliable results between the pre-test and post-test. Items with coefficients below 0.5 were removed from the final questionnaire. However, all but two items had high correlation coefficients which ranged from .68 to 1.0 . Two items, importance of the decision to the goals of the health unit, and the extent to which research evidence was analyzed prior to making a decision, had coefficients of .5 and .45 respectively, and therefore were not included in the final questionnaire. The data collected from this health unit were only used to measure the reliability 
and face validity of the instrument and were not included in further analysis. The questions included in both Appendices 1 and 2 were modified from previous diffusion of innovation and reseârch utilization studies (Champion et al., 1989; Burns et al., 1997; Cockerill et al., 1996; Dean et al.. 1993; Funk et al., 199 la; Funk et al., 199 lb; Kaluzny et al., 1974; Kimberly et al.. 1981; Meyer et al., 1988; Pettengill et al., 1994; Rogers, 1983; Veeramah, 1995).

The other two instruments, Appendices 3 and 4, were used to collect data on characteristics of the innovation and individuals during the OHCEN study baseline and followup surveys. The reliability and face validity of both questionnaires were discussed in chapter 1 of this dissertation.

All of the independent and dependent variables were measured as individual items using a number of Likert response items or continuous variables. None of the variables have been scaled into constructs at this time. However, further discussion with respect to not scaling the independent variables will be discussed later in the Methods section.

\subsection{Quantitative Analysis}

The analysis included simple descriptive summaries such as means, frequencies, and where applicable standard deviations for all study variables. A principal component analysis was conducted on the characteristics of the innovation to determine the feasibility of including factors of the innovation as opposed to individual independent variables in the bivariate and multivariate analyses. As a result of the large number of independent variables included in the analyses, a four step analysis was conducted for both the multiple logistic regression and multiple linear regression analyses. This included an analysis of variance, a bivariate correlation analysis, multiple regression by type of characteristic and the full multiple regression model. The process used for both the logistic and linear regression will now be discussed in greater detail. 
Analyses of variance (one way ANONA) were then conducted on each dependent variable. The Bonfertoni adjustment for multiple comparisons produced a very small significance level of 0.00002 , and therefore was not used in the analysis, as this would have resulted in none of the independent variables being significant in the analyses of variance. Therefore, the significance level was maintained at 0.10 , although some of the variables may have been significant due to chance. Since this was an exploratory study, a decision was made to be more inclusive at this time. All variables observed to be significant in the analysis of variance at $p<0.10$ were then examined in a bivariate correlation analysis. Independent variables that were significantly $(p<0.05)$ correlated with the dependent variables, had correlation coefficients exceeding 0.2, and did not have significant correlation coefficients with other independent variables above 50 , were then included in a multiple linear regression according to type of characteristic. For example all the significant organizational characteristics were included in one linear regression model, along with separate multiple regressions for the environmental, individual and innovation characteristics. This has been suggested as an important step in model development (Feinstein, 1996; Hosmer et al., 1989; Norusis, 1997). In instances where there was only one significant variable from a category (i.e. environmental characteristics), the variable proceeded directly to the full multiple regression model if its correlation coefficient in the bivariate analysis was statistically significant at $p<0.05$ and above 0.2 . All of the significant variables from this first set of multiple regression analyses were then included in the full multiple regression analysis.

The purpose of this study was to identify characteristics that were associated with the use and influence of the systematic overviews. Therefore, the appropriate statistical analyses to use were regression analyses, which would identify those variables that predicted the outcome of 
interest. Since overall use of the systematic overviews was measured as a dichotomous variable the most appropriate statistical procedure was multiple logistic regression (Feinstein, 1996; SPSS Inc, 1998). As well, since the remaining five outcomes were measured using a Likert scale, the appropriate statistical procedure for these outcomes was multiple linear regression (Feinstein, 1996: SPSS Inc, 1998).

\section{Principal Component Analysis}

A principal component analysis with Varimax rotation was conducted with the 23 characteristics of the innovation, with the intent of replacing the large number of independent variables with a smaller, more manageable number of broad factors. The results are presented in Table 2. The criteria used to retain factors for further analysis were taken from work based on factor component scale development (Shortell et al., 1991). The two criteria used to retain factors were 1) eigenvalues greater than 1.0; and 2) each criterion had a primary factor loading of 0.4 .

Although there were initially five factors identified from this analysis that exceeded eigenvalues of 1.0 , and the majority of items had a primary factor loading above 0.4 , there were a number of reasons why the factors of the innovations did not present as being the most usetul and informative measures to use in the multivariate analyses in this dissertation. First, this was the first time these variables were measured in the public heaith setting. If they were all collapsed into a small number of factors, little would be gained in terms of the individual relationship each has or does not have on research utilization, among public health decisionmakers. In addition, since the main objective of this study was to examine the relationships between these variables and research utilization outcomes with the intent of using this analysis to develop specific dissemination strategies, then creating factors of the innovation characteristics could hinder rather than facilitate this objective. An example of this was made clear when a 
number of the multiple linear regression analyses were conducted comparing the models when factors of the innovation were used versus single innovation characteristics. In each case, the model containing the single innovation characteristics produced higher adjusted $\mathbf{R}^{2}$ values. These analyses also demonstrated that in all cases, there were some innovation characteristics that were allocated to a factor that were found to be significant in the multiple linear regression, but when the innovation characteristics were tested as individual variables, many times they were not found to be statistically significant. Therefore, it was confusing as to which analyses were more appropriate to use. Given the importance of identifying specific characteristics associated with research utilization in public health, it was decided that the innovation factors from the principal component analysis would not be used in this analysis, recognizing that there were some statistical issues with using such a large number of independent variables. Future studies in this field may lend themselves to examine these innovation factors more closely. Therefore, all 23 characteristics of the innovation were assessed individually.

\section{$\underline{\text { Logistic Regression Modeling }}$}

The multiple logistic regression procedure was conducted using a backward elimination approach with the likelihood ratio test for determining elimination. Once all significant variables were identified in the final model, interaction terms were tested for their effect. Interaction terms were chosen based on findings from the research utilization and diffusion of innovations literature discussed in Chapter 3. The testing of interaction terms based on those variables remaining in the final model was supported by Feinstein, (1996). In addition, interaction terms were limited to pairs of independent variables. Once all significant independent variables were identified and inciuded in the model, diagnostics of the model fit were conducted. A variety of diagnostic tests were used to identify outliers beyond three standard deviations from the mean. 
These included normal probability plots of measures related to the observed and expected deviances, as well as measures related to the observed data versus the deviance from normal values. A number of scatter plots were used to identify outliers by plotting the computed standardized residuals for each case, the computed leverage for each case, as well as the influence each case had on each regression coefficient (dfbeta). Common outliers (decisionmakers) were identified using these plots and then the data for these outliers were removed from the dataset. The logistic regression analysis was rerun to produce the final regression coefficients.

The output from this final regression model was then assessed for a decreased $-2 \log$ Likelihood value, improvements in the Hosmer and Lemeshow Goodness of Fit Test score, and greater accuracy in the classification table. All significant variables, or those that would result in significant changes to the regression coefficients if removed, were retained for the final logistic regression model. Once the final model was identified, the final diagnostic used to determine the

overall fit of the model was the C-statistic. The C-statistic measures the proportion of times the model assigned a higher probability of the outcome occurring when it actually did occur versus not occurring. (Norusis, 1997). A C-statistic below 0.5 indicates that the logistic model is not effective in accurately identifying those cases in which the outcome occurred beyond what would be expected by chance alone. The closer this value is to 1.0 , the greater the predictive power of the model.

\section{Multiple Linear Regression}

A similar approach was used in the multiple linear regression analyses as was described for the logistic regression. All independent variables were initially assessed in an analysis of variance, and all those with a $p$ value of $<0.10$ were then analyzed in a bivariate correlation 
analysis. Those variables significantly correlated with the outcome at the $p<0.05$ level with correlation coefficients above 0.2 , and were not highly correlated $(>.5)$ with other independent variables were then analyzed by category in a multiple linear regression model. This occurred in all cases except when only one variable was found to be significant in a category. In this instance if the correlation coefficient between the independent variable and the outcome was significant and greater than 0.2 in the bivariate analysis, then that variable proceeded directly to the full multiple regression analysis. Variables with a $\mathrm{p}$ value $<0.05$ in the multiple linear regression by type were then included in the full multiple linear regression analysis. The multiple linear regression analyses were conducted using the backward elimination procedure to identify potentially significant variables. The same process for selecting interaction terms in the multiple logistic regression analysis was used for the multiple linear regression analysis. If these interactions were found to be statistically significant they were included in the full model. The final multiple linear regression model forced all variables with main effects into the model.

A number of diagnostic tests were conducted to determine the overall fit of the model and identify outlying cases. First the computed leverage values were assessed and any cases with values exceeding 0.5 were assessed for removal from the dataset. The cut off point of 0.5 is supported in (SPSS Inc, 1998). Outliers were also identified by plotting the computed studentized deleted residuals against the standardized predicted values, the standardized predicted values against the observed values for the dependent variable (heteroscedasticity), and the studentized deleted residuals against the centered leverage value. Finally, individual plots of the observed data for the dependent variable and each of the independent variables included in the model were used to identify outlying cases. Data from extreme outliers or outliers common among the diagnostic tests were then removed from the dataset, and the multiple linear 
regression model was rerun. Multicollinearity was assessed only in the final multiple linear regression model by assessing the tolerance values for each of the independent variables in the final model. Indicators of model improvement included a reduction in the residual sum of squares and in the standard error of the estimate. 


\subsection{Results}

\subsection{Characteristics of the Respondents and Public Health Units}

\section{Response Rates}

Thirty-five of the $41(85.4 \%)$ public health units agreed to participate in this study. During 1998 six public health units, (City of Toronto, City of Etobicoke, Borough of East York, City of North York, City of York and Scarborough,) were amalgamated to form the Toronto Public Health Lnit. However, since data were initially collected in the OHCEN study (1996) from decision-makers at each of the six health units and the amalgamation had not been completed at the time the data for the current study was collected, the six public health units were examined separately. Figure 3 depicts how 141 decision-makers from the original 205 remained to participate in this study.

\section{Figure 3: Study Sample}

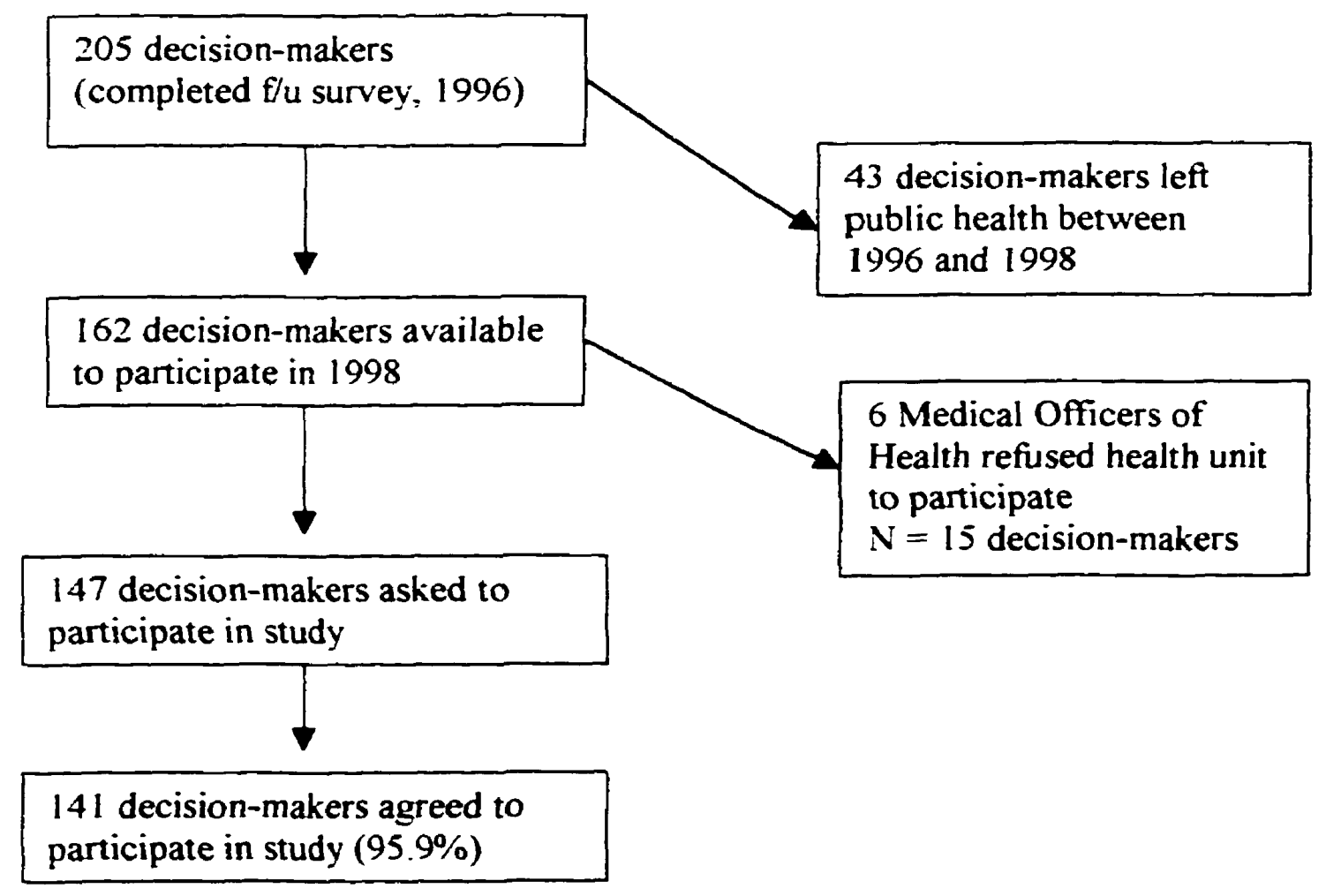


Therefore, there was a total of 141 respondents out of a possible 147 decision-makers who participated in the study, resulting in a participation rate of $95.9 \%$. There were no statistically significant differences between those who participated in this study and those who had left public health during the two year period on characteristics such as age, position, years in current position, years in public health and years since graduation.

Although there were 141 decision-makers included in this study, there was only complete data for on the independent variables for 88 cases. This occurred as a result of the data collection methods used during the OHCEN study in 1996. For example, decision-makers who had not used a systematic overview between the baseline and follow-up surveys were not asked to provide data on characteristics of the innovation, such as the perceived ease of use, relevance to decisions, and appropriateness of the timing for receiving the overviews. This was not considered to be missing data since it was not appropriate to collect data on these cases. and therefore no imputation for missing values was calculated. Therefore, the multiple logistic regression analysis was conducted using the 88 cases for which complete data existed. As well, for the multiple linear regression analyses, there were cases for which data was not available, depending which independent variables were included in the final model, which resulted in a different number of cases being used for each of the five multiple linear regression analyses.

The number of respondents from each health unit varied between one to fifteen. The mean number of respondents per health unit was 4.2 , and the average participation rate from each health unit was $95.0 \%$. Five point seven percent of public health units ( 2 health units) had only one participant, $22.9 \%$ (8) units had two respondents, $25.7 \%$ (9) had three respondents, 
$11.4 \%$ (4) had four respondents, and $25.7 \%(9)$ had five or six respondents. Three other health units had seven, nine and fifteen respondents respectively.

Independent Variables

The theoretical framework discussed earlier in this dissertation, based on the results of decades of research in the areas of diffusion of innovations, research utilization and dissemination, identified four main categories of characteristics that are associated with research utilization. This includes characteristics of the 1) organization, 2) environment, 3) innovation and the 4) individual. A number of independent variables from each of these categories were examined in this study, the data for which will now be presented. The means and where applicable the standard deviations for all independent variables are summarized in Table 3. Only those results considered to be of importance or unusual will be discussed in detail below.

\section{Organizational Characteristics}

The fifteen organizational characteristics addressed organizational structure issues such as size and complexity, as well as issues related to respondents' perceptions of how the organization functioned. The fifteen organizational characteristics addressed organizational structure issues such as size and complexity, as well as issues related to respondents' perceptions of how the organization functioned. The fifteen characteristics included 1) the number of full time equivalent staff; 2) the urban/rural mix of the population served; 3) the number of divisions; 4) the number of programs; 5) the number of levels of workers; 6) involvement in research activities; 7) the number of ongoing research projects in the health unit; 8) teaching health unit status; 9) adherence to policies and procedures; 10) adequate financial resources; 11) value organization placed on research evidence; 12) ongoing training in research methods and critical 
appraisal; 13) mechanisms to facilitate the transfer of new information into the organization; 14) the extent to which information is routinely searched for before making decisions; and 15) the importance routinely placed on research evidence to make decisions.

With respect to the organizational context variables, the mean number of full time equivalent personnel employed in the 35 public health units was 131 , with a minimum of 35 and a maximum of 322. The number of divisions such as Environmental Health and Nursing ranged from two to eight with a mean of four divisions, while the number of public health programs provided ranged from a low of 14 to a high of 42 . The majority of health units served an urban population and almost all of the health units $(91.5 \%)$ reported being involved in some form of research activity.

Respondents overwhelmingly perceived that their health unit strictly adhered to policies and procedures with $95.1 \%$ strongly or moderately agreeing with this statement. In addition $90.0 \%$ of respondents strongly or moderately agreed that their health unit valued the use of research evidence for making decisions, while $65.3 \%$ strongly or moderately agreed that limited organizational financial resources were a barrier to using research evidence. The majority of respondents $(61.7 \%)$ perceived that information systems existed within the health unit that facilitated the transfer of new information into the health unit, while only $49.7 \%$ of respondents perceived that ongoing training in research methods and critical appraisal skills were provided to staff.

In terms of organizational decision-making processes, respondents generally agreed that their health units routinely extensively searched for information before making decisions. However, they did not perceive that their health units placed a great deal of importance on using this retrieved research evidence in making decisions. For example, the majority of respondents, 
$67.9 \%$, rated their health unit search behaviours as extensive, whereas only $45.0 \%$ of respondents reported research evidence as being very important to their decision-making. An analysis of the within health unit variation of perceptions of these organizational characteristics versus between health unit variation demonstrated that between group variation was greater than within group variation for the first four variables, but not for the final three variables.

\section{Environmental Characteristics}

The six environmental characteristics addressed issues related to respondents' perceptions of how their environment influenced their decisions to use research evidence. The six characteristics included provincial and municipal regulations and legislation, network embeddedness, the medical officer of health's influence over the local board of health and local politicians, and whether the local board of health and local politicians were influenced by research evidence. The results demonstrated that respondents perceived three of the environmental characteristics to influence their decision-making.

Almost all of the respondents $(94.4 \%)$ perceived that provincial and municipal regulations had a great deal of impact on their decision-making. Even though $83.7 \%$ of respondents perceived that their medical officer of health had a great deal of influence over the local board of health, only $42.5 \%$ perceived their medical officer of health to have a great deal of influence over local politicians. Respondents generally did not perceive the local board of health or local politicians to be influenced by research evidence. An analysis of the within health unit variation versus the between health unit variation for these variables indicated that the between group variation was greater than the within group variation for all but one of the variables, which was collaboration with local agencies. 
Characteristic of the Innovation

The 23 characteristics of the innovation assessed respondents' perceptions of the relative advantage, compatibility, complexity and bandwagon effects of the systematic overviews. Relative advantage was measured by asking respondents about their perceptions of the priority that should be given to conducting systematic overviews both before and after receiving the overviews, as well as their perceptions of whether systematic overviews could overcome barriers such as limited availability of literature, not enough time to review literature, limited critical appraisal skills, limited financial resources, lack of research relevance to decisions being made, unsupportive work environments to using overviews and lack of decision-making authority both time $\mathrm{l}$ (T1) and time 2 (T2). Compatibility was measured by asking respondents about their perceptions of the weight that is given to using research evidence for making decisions, their perception of how much weight research evidence should be given, whether the systematic overview was relevant to decisions being made, and the appropriateness of the timing for receiving the overviews. Complexity was measured by asking respondents about their perceptions of how easy the overviews were to use. A bandwagon effect was measured in two ways. The first was respondents' perceptions of the percentage of public health units using systematic overviews to make program decisions, and the second was their perception of the percentage of colleagues, in the same position as themselves, who were using overviews to make program decisions.

Perceptions of the relative advantage of the systematic overviews were very positive before the overviews were disseminated and even more positive after the overviews were disseminated. However, this difference was not statistically significant. For example, $75.2 \%$ of 
respondents perceived that the priority that should be given to conducting systematic overviews, on a scale of "low", "middle", "high" and "top", should be high or top, before they received the overviews. After receiving the systematic overviews $87.7 \%$ of respondents perceived that the priority that should be given to conducting overviews was "high" or "top".

Similar findings were also reported when respondents' perceptions of whether systematic overviews could overcome the barriers identified previously were assessed before and after receiving the systematic overviews, with respondents generally having more positive perceptions of the overviews after receiving them, although this difference was not statistically significant. Respondents' perceptions of whether systematic overviews could decrease the barrier of limited critical appraisal skills was the only barrier that decreased after the overviews were disseminated, although the decrease was insignificant. For example, $83.7 \%$ of respondents perceived that systematic overviews probably would or definitely would overcome the barrier of limited critical appraisal skills before receiving the overviews, while $83.1 \%$ reported this after receiving them. Although the majority of respondents $(53.9 \%)$ did not perceive that systematic overviews could overcome the barrier of limited financial resources at $T 1$, less than half of these respondents $(23.3 \%)$ continued to perceive this after receiving the overviews.

The public health decision-makers perceived that the systematic overviews were compatible with their values, beliefs and environments. For example, 22.0\% of respondents perceived that research evidence was given $60.0 \%$ or more weight in making decisions, while $18.4 \%$ perceived that research evidence was given less than $20.0 \%$ weight to make decisions. When asked how much weight research evidence should be given, $61.7 \%$ responded that research should be given $60.0 \%$ or more weight in decision-making and only $1.4 \%$ responded that it should receive less than $20.0 \%$ weight. In addition $89.9 \%$ perceived the relevance of the 
overviews to be either "good" or "excellent". The perception of the appropriateness of the timing for receiving the systematic overviews was slightly less positive, although still high, with $80.6 \%$ of respondents reporting the timing as "good" or "excellent".

Respondents, on the whole, had positive perceptions with respect to the complexity of the systematic overviews. For example, $92.6 \%$ of respondents perceived the overviews as being "good" or "excellent" for being easy to use, The final two measures of perceptions of the innovation measured the bandwagon effect of the systematic overviews. Some unexpected results were observed from these two variables. For example, when asked what percentage of public health units in Ontario had used the systematic overviews in the past two years to make decisions, $30.0 \%$ stated they could not answer this question as they had no way of knowing which health units were using the overviews or not. Those who answered this question reported use by other health units as ranging from 3.0\% to 100\%. As a group, respondents perceived that less than half of the public health units in Ontario used the systematic overviews to make decisions. Similar results were observed when respondents were asked what percentage of their colleagues had used the systematic overviews to make decisions, suggesting that perceived use of the overviews was not high across the province.

\section{Individual Characteristics}

There were fourteen individual characteristics examined in this study such as age, years in public health, years in current position, and a number of variables related to respondents' perceptions of barriers to using research evidence to make program decisions.

Respondents were classified into four distinct positions of which $54.6 \%$ were program managers, $27.7 \%$ were program directors, $15.6 \%$ were medical officers of health and $2.1 \%$ were 
associate medical officers of health. Respondents had been in public health for a mean of 17.9 years, had a mean age of 50 years, and the number of years since the most recent graduation was 13.8 years.

The majority of respondents (75.9\%) reported they had access to a research consultant who assisted them in interpreting research results, and had direct access to on-line database searching at their public health unit $(64.5 \%)$. Almost all respondents $(92.9 \%)$ had retrieved one or more journal articles in the past month and almost half of the respondents had read between $50-100 \%$ of these retrieved articles. The mean number of days spent at conferences or workshops in the past year was 5.7 days.

There were five additional individual characteristics for which data were collected. Three were related to the respondent's perceptions of barriers associated with using research evidence to make program decisions, and the final two were related to prior use of systematic overviews or an expectation to use them in the future. Inadequate financial resources were perceived to be the greatest barrier to using research evidence for decision-making, with $47.5 \%$ of respondents stating this was a serious to very serious barrier. In terms of previous use of systematic overviews. $39.7 \%$ stated they had used a systematic overview in the past to make a decision and $84.7 \%$ stated they expected to use a systematic overview in the future.

\section{Dependent Variables}

Overall Uise of the Systematic Overview

Respondent's use of the systematic overviews was initially assessed using the dichotomous variable of use versus no use. However before respondents were asked if they had used at least one of the systematic overviews in the previous two years, they were asked to choose one of the overviews they felt comfortable answering questions about concerning its 
overall use and its influence on the five public health decisions. When asked which overview they would like to discuss $42.0 \%$ chose the Home Visiting overview, $19.3 \%$ chose the Community-Based Heart Health overview, $18.5 \%$ chose the Parent Child overview, $11.8 \%$ chose the Community Development overview and $8.4 \%$ chose the Adolescent Suicide Overview, as presented in Table 4.

Respondents were asked if they had used the overview they had chosen to discuss in the past two years to make a decision. The term "use" indicated that the systematic overview might have been used on its own to make a program decision, or in combination with other pieces of evidence. as defined in the literature review. In total $63.1 \%(89)$ of respondents included in this study stated they had used at least one of the systematic overviews, in some way, in the past two years to make a decision, as is presented in Tabie 5. Program directors were most likely to have used one of the systematic overviews in the past two years and were closely followed by program managers, as is summarized in Table 6.

The Influence of Systematic Overviews on Five Public Health Decisions

Table 5 illustrates the mean and standard deviations of the perceived influence of the systematic overviews on decisions related to program planning, program justification, program evaluation. policy development and staff development. Using a five point Likert scale where a rating of 1 means "Not at All", and 5 means "A Great Deal", respondents were asked to comment on the extent to which they perceived the systematic overview had influenced the above mentioned decisions. Overall, respondents perceived the systematic overviews as having the greatest influence on decisions related to program planning and program justification, and the least amount of influence on program evaluation, policy development and staff development 
decisions. A summary of the perceived influence of the systematic overviews on all five decisions is presented in Table 7.

The Influence of Systematic Overviews on Program Planning

Many of the respondents perceived systematic overviews to have a great deal of influence on decisions related to program planning with $41.3 \%$ of respondents rating their influence as a four or a five on a five-point Likert scale. Only $13.8 \%$ of respondents rated the influence of the systematic overviews as a one or two, while the majority, $44.8 \%$, rated the influence as being in the middle between "Not at all" and a "Great Deal".

The Influence of Systematic Overviews on Program Justification

Just under half of the respondents $(49.4 \%)$ perceived the influence the systematic overviews had on program justification decisions to be a four or five on the rating scale, while $19.5 \%$ rated the influence as being a one or two, and $31.0 \%$ rated it in the middle at three.

Influence of the Systematic Overview on Program Evaluation

Whereas the majority of respondents perceived systematic overviews to have a moderate to high degree of influence over program justification and program planning decisions, this did not hold true for program evaluation, policy development and staff development decisions. In fact, only $17.4 \%$ of respondents perceived the systematic overviews as having a "Great Deal" of influence on program evaluation. The majority of respondents $(45.3 \%)$ perceived the influence as being in the middle between "Not at all" and a "Great Deal", while another $37.2 \%$ reported a rating of one or two, indicating little to no influence on program evaluation decisions. 
The Influence of Systematic Overviews on Policy Development

Respondents perceived systematic overviews to have the least amount of influence on decisions related to policy development. For these decisions $44.2 \%$ of respondents perceived the systematic overviews to have little or no influence on these decisions, while another $39.5 \%$ perceived the influence to be in the middle of the rating scale. Only $16.3 \%$ of respondents rated the influence of the systematic overviews on policy development decisions as a "Great Deal".

The Influence of Systematic Overviews on Staff Development

Very similar results were obtained for the perceived influence of the systematic overviews on decisions related to staff development as were for policy development. For example, the majority of respondents $(40.2 \%)$ rated the influence as being a one or two, while another $39.0 \%$ rated the influence as being in the middle between "Not at all" and a "Great Deal". As well, only $16.1 \%$ rated the influence of the systematic overviews on staff development decisions as a four or five on the rating scale.

\subsection{Bivariate Correlation Anslysis: Dependent and Independent Variables}

The results of the bivariate correlation analysis between all the independent variables and the six dependent variables are presented in Table 8 . This summary table demonstrates that different independent variables were statistically significantly associated with each of the six independent variables, although some overlap was observed. For example the value the organization placed on research was significantly correlated with a number of the outcomes as well as several characteristics of the innovation. 


\subsection{Use of the Systematic Overviews}

\section{$\underline{\text { Results of the Analysis of Variance }}$}

There were a total of 49 analyses of variance conducted when the dependent variable was overall use of the systematic overviews. Eight variables were statistically significant in explaining the observed variation in the use of the systematic overviews. These results are presented in Table 9. Six of the eight significant variables were related to perceptions of the characteristics of the innovation, while the remaining two variables were related to characteristics of the individual.

Respondents' perceptions of the characteristics of the systematic overviews (innovation) proved to be important variables in explaining the variance between those who used the systematic overviews and those who did not. Two of the significant variables measured the perceived compatibility of the innovation, while three others were measures of relative advantage, and the one was related to complexity. The first significant compatibility characteristic, which was measured before the systematic overviews were received, was the weight given to research evidence to make decisions. The second compatibility characteristic was the respondents' perceptions of the appropriateness of the timing for receiving the overviews, which was measured at T2. The three significant characteristics related to relative advantage were all measured at $\mathrm{T} 2$ and included respondents' perceptions that the systematic overviews would overcome the barrier of limited critical appraisal skills, the perception that the overviews would overcome the barrier of not having enough time to review literature, and the perception that systematic overviews would overcome the barrier of unsupportive work environments for using research evidence in decision-making. The remaining significant complexity characteristic was also measured after the systematic overviews had been received and measured respondent's perceptions of how easy the systematic overviews were to use. The 
two significant characteristics of the individual were the respondent's position in 1996, and whether the respondent expected to use the systematic overview in the future.

Results of the Bivariate Correlation Analysis

The Pearson correlation matrix of the eight significant variables is presented in Table 10. Although some of the variables were significantly correlated at the $p<0.05$ and 0.01 levels, none of the correlation coefficients were greater than .5. Although this would have allowed all eight variables to be included in the full logistic regression model, the small and insignificant correlation coefficients between overall use and three of the independent variables resulted in their removal from further analysis. The three variables removed from further analysis were the weight given to systematic overviews to make decisions, the perceived appropriateness of the timing for receiving the overviews, and the perception that systematic overviews would overcome the barrier of an unsupportive work environment at T2. The five remaining variables were judged to be appropriate for inclusion in the full multiple logistic regression analysis.

\section{Results of the Multiple Logistic Regression}

All five of the remaining significant independent variables were included in the multiple logistic regression model which was conducted using the 88 cases with complete data. Using the backward elimination procedure described in the Methods section, four variables remained in the model. Three cases were identified as outliers beyond three standard deviations from the mean. Before their removal, the effect of the removal of these cases on the regression coefficients was assessed. After it was determined that the effect was small these outliers were removed from further analysis. After the removal of the three outlying cases the model was rerun, and no further variables were removed from the model. The four variables remaining in the final logistic regression model included two characteristics of the individual and two characteristics of the 
innovation. The two individual characteristics were expecting to use the systematic overviews in the future, and the respondent's position at baseline. The respondents' position was a categorical variable with two dummy variables, one for program directors and another for program managers. The reference group was the combined cells of medical officers of health and associate medical officers of health, due to a small cell size for associate medical officers of health. The two characteristics of the innovation included the respondents perception of how easy the systematic overviews were to use, and the respondents' perception that systematic overviews would overcome the barrier of limited critical appraisal skills. The results of the final logistic regression model are summarized in Tables 11 and 12.

The -2 Log Likelihood of the final regression model was 59.059, which was decreased from 90.327 for the model containing only the constant. The Hosmer and Lemeshow Goodness of Fit Test produced a Chi-Square value of 6.05 and a p value of .5907 , which indicated a strong fit for the model to the data. The Classification Table results demonstrated a high overall percent correct prediction rate of $80.05 \%$, with the model accurately predicting that the overviews were used $93.3 \%$ of the time, and accurately predicting the overviews were not used $56.2 \%$ of the time.

The odds ratio of the likelihood of use demonstrated that as respondents perceived systematic overviews to be more effective in overcoming the barrier of limited critical appraisal skills at T2, the likelihood of using the systematic overviews increased significantly. The results indicated that respondents were 3.49 times more likely to use a systematic overview when they perceived that the overviews would overcome the barrier of limited critical appraisal skills as opposed to when they did not. 
The Beta coefficient for the variable 'ease of use' demonstrated that the less respondents perceived the overviews to be easy to use, the less likely they were to have used it. Therefore, respondents who rated the ease of use of the systematic overviews as poor were only .33 times as likely to have used the overviews as compared to those who rated the overviews as excellent.

The same scenario was observed when respondents were asked if they expected to use the systematic overview in the future. For example, respondents who stated they did not expect to use the systematic overviews in the future were only 0.189 times as likely to have used the overviews, as compared to those who stated they did expect to use the overview in the future.

With respect to the relationship between the respondent's position at baseline and the use of the systematic overview two years later, the results demonstrated that position significantly predicted overall use. Medical and associate medical officer of health were the reference group for this categorical variable, which identified how likely program managers and program directors were to use the systematic overviews in comparison to medical and associate medical officers of health. Program directors were 12.65 times more likely to have used a systematic overview as compared medical and associate medical officers of health, while program managers were II.3l times more likely to have used an overview. Both program directors and managers were significantly more likely to have used an overview since being disseminated than medical and associate medical officers of health.

The following interaction terms were tested for effect in the model: position*critical appraisal skills; position*expect to use in the future; position*easy to use; critical appraisal*easy to use; critical appraisal*expect to use; and expect to use*easy to use. None of the interaction terms tested in the final model were observed to be statistically significant. The normal probability plot and detrended plot of the deviance was normally distributed, suggesting that the 
model was a good fit for the data. The final measure of model fit was the $\mathrm{C}$ statistic. The results demonstrated that the model accurately predicted the use of the systematic overviews $93.0 \%$ of the time between pairs of cases where one case actually used the overview and the other did not.

\subsection{The Influence of Systematic Overviews on Program Planning \\ Results of the Analysis of Variance}

The results of the 49 analyses of variance conducted when the dependent variable was the influence the systematic overviews had on program planning decisions are presented in Table 13 . The results identified nine variables that were statisticaily significant in explaining the variation in the influence the systematic overviews had on program planning decisions. The nine variables included three organizational characteristics, three individual characteristics and three innovation characteristics.

The three significant organizational characteristics included two variables related to perceptions of the organization and one variable related to size. The first variable was the respondents' perception of the value the organization placed on research evidence in decisionmaking, while the second was the respondent's perception of whether there was ongoing training in research methods and critical appraisal provided to staff by the organization. The third organizational characteristic was the number of full time equivalent employees in the health unit.

The three significant innovation characteristics, measured at $\mathrm{T} 2$, included two measures of relative advantage and one measure of compatibility. The two measures of relative advantage included the perception that systematic overviews would overcome the barrier of limited critical appraisal skills, and the perception that systematic overviews would overcome the barrier of not having enough time to review the literature. The one measure of compatibility was the perception that the overviews were relevant to decisions being made. 
The three statistically significant individual characteristics measured the respondent's age, the percentage of articles retrieved in the past month, and the percentage of retrieved articles that had been read in the past month.

\section{Bivariate Correlation Analysis: Program Planning}

The Pearson correlation matrix of the nine significant variables is presented in Table 14 . When the correlation coefficients between the dependent and independent variables were assessed. two were not statistically significant. Therefore, the number of full time equivalent staff and the percentage of articles retrieved in the past month were removed from further analysis. There were a number of significant correlation coefficients observed at the $p<0.05$ and 0.01 levels between the remaining seven independent variables. However, all but one of the correlation coefficients were below .35 , and one was .437 . Although there were statistically significant correlation coefficients among these independent variables, they were considered to be small enough to warrant the inclusion of all seven variables in the multiple linear regression model for each of the categories of characteristics, such as organizational, individual, and innovation characteristics.

\section{Multiple Linear Regression of the Influence of Systematic Overviews on Program Planning -}

\section{Organizational Characteristics}

The two remaining significant organizational characteristics were then included in a multiple linear regression model that considered only the organizational characteristics. Only one of the two organizational characteristics, the value the organization placed on research evidence for decision-making, remained in the model after using the backward elimination procedure. This characteristic was then retained for the full multiple linear regression model. 
Multiple Linear Regression of the Influence of Systematic Overviews on Program Planning Individual Characteristics

Both of the individual characteristics remained significant when included in the multiple linear regression model containing only individual characteristics. They were the percentage of retrieved articles read in the past month and age category. Both of these variables were retained for the full multiple linear regression model.

Multiple Linear Regression of the Influence of Systematic Overviews on Program Planning Innovation Characteristics

There were three innovation characteristics included in this multiple linear regression model of characteristics of the innovation. The backward elimination procedure eliminated all but one of them. The remaining characteristic of the innovation was the perceived relevance of the systematic overviews to decisions being made at T2. Therefore, only this one characteristic of the innovation was retained for the full multiple linear regression model.

Significant Predictors of Systematic Overviews Influencing Program Planning Decisions: Results of the Multiple Linear Regression

The full multiple linear regression model was conducted on 66 cases and the four significant variables (the value the organization placed on research evidence, age, percentage of retrieved articles read in the past month, and the perceived relevance of the systematic overviews to decisions being made), that were identified from the multiple linear regressions for each category. Using a backward elimination procedure one of the four variables, age, was removed 
from the model. Diagnostic tests on the final model did not identify any outlying cases. The results of the final model are summarized in Tables 15 . The explanatory power of this significant multiple regression analysis was moderately high, as the adjusted $R^{2}$ was .264 . This means that the final model explained almost $26.4 \%$ of the variation in the observed influence of systematic overviews on program planning decisions.

The final regression model contained one organizational characteristic, one individual characteristic. and one innovation characteristic. As demonstrated in Table 15. the perceived relevance of the systematic overviews at $\mathrm{T} 2$ to decisions being made, had the greatest impact on whether the overviews were perceived as influencing program planning decisions. The standardized Beta coefficient of .379 indicated that the more relevant systematic overviews were perceived to be, the greater the perceived influence they exerted on program planning decisions. The second most influential characteristic was the perceived value the organization placed on research evidence. Again the standardized Beta coefficient of .278 demonstrated that the more respondents perceived their organizations to value evidence, the greater the perceived influence the systematic overviews had on program planning decisions. The final characteristic in the model, was the percentage of retrieved articles read in the past month. Although this variable exerted the least impact on the dependent variable it still represented an important finding. The Standardized Beta coefficient of .212, demonstrated that the more respondents read on a monthly basis, the greater the perceived influence the systematic overviews had on program planning decisions.

Finally, the effects of the following interaction terms were tested: organizational value*relevance T2; organizational value*articles read: and articles read*relevance T2. None of these interaction terms were significant in the model. A normal probability plot of the observed 
versus expected probabilities identified a strong linear relationship between the observed and expected values. The tolerance values measured multicollinearity among the independent variables in the final model and ranged from .975 to .996 . This illustrated that multicollinearity was not a problem for the three variables included in the final multiple linear regression model.

\subsection{Influence of Systematic Overviews on Program Justification}

\section{Results of the Analysis of Variance}

The 11 statistically significant independent variables from the 49 analyses of variance conducted on the perceived influence of systematic overviews on program justification decisions, are presented in Table 16. Five of the eleven significant variables were organizational characteristics, three were environmental, two were individual and one was a characteristic of the innovation

The five significant organizational characteristics were: the perceived value the organization placed on research evidence to make decisions; perceived adequate financial resources; the perception that ongoing training in research methods and critical appraisal was provided to staff: the number of full time equivalent staff; and the extent to which information was routinely searched for before making program decisions.

The three statistically significant environmental characteristics were: respondents' perception that their health unit collaborated with local agencies to make decisions; the perception of whether the medical officer of health influenced the local board of health; and the perception of whether local politicians were influenced by research evidence.

The two individual characteristics were the number of retrieved articles read in the past month and expecting to use the systematic overviews in the future. 
The only significant characteristic of the innovation was the perception that systematic overviews would overcome the barrier of not having enough time to review literature, measured at Time 2.

\section{Bivariate Correlation Analysis: Program Justification}

The bivariate correlation matrix presented in Table 17 demonstrated that many of the eleven variables were statistically significantly correlated at the $p<0.05$ and 0.01 levels. The correlations were strongest among those variables within the same category, such as between organizational characteristics, but some significant associations were also observed between variables from different categories, such as organizational and environmental ones. However, even though variables were statistically significantly correlated, none of the correlation coefficients exceeded .437 , which was considered low enough to warrant the inclusion of all eleven variables. However, further examination of the correlation coefficients between the dependent and independent variables, illustrated that two of the eleven variables, the number of full time equivalent staff, and the perception of whether the medical officer of health influenced the local board of health were small and not statistically significant. Therefore, both of these variables were removed from further analysis. As well, since the innovation characteristic was the only significant variable in its category, and because its correlation coefficient with the dependent variable was significant at $p<0.05$, and above 0.2 , it proceeded directly to the full multiple linear regression analysis. In addition the remaining eight significant variables were retained for inclusion in the multiple linear regression analyses by type of characteristic. 
Results of the Multiple Linear Regression of the Influence of Systematic Overviews on Program Justification - Organizational Characteristics

The four significant organizational characteristics were included in a multiple linear regression model of only organizational variables. Using a backward elimination procedure, one of the four organizational characteristics, perceived adequate financial resources, was removed from the model. The three remaining organizational characteristics were the perceived value the organization placed on research evidence, the perception of whether ongoing training in research methods was provided to staff and the extent to which information was routinely searched for before program decisions were made.

Results of the Multiple Linear Regression of the Influence of Systematic Overviews on Program Justification - Environmental Characteristics

Only one of the two environmental characteristics was significant in this multiple linear regression analysis. This was the perception of whether local politicians were influenced by research evidence. This variable was retained for use in the full multiple linear regression model.

Results of the Multiple Linear Regression of the Influence of Systematic Overviews on Program Justification - Individual Characteristics

Both of the individual characteristics, the percentage of retrieved articles read, and expecting to use the systematic overviews in the future remained in the multiple linear regression model when tested together in one model. Therefore, both variables were retained for further analysis in the full multiple linear regression model. 
Significant Predictors of Systematic Overviews Influencing Program Justification Decisions:

\section{Results of the Multiple Linear Regression}

The full multiple linear regression model was conducted on 73 cases and the seven significant variables identified from the multiple linear regressions from each category of characteristics. These were perceived value the organization placed on research evidence, perception of ongoing training in research methods, perception of the extent to which information was routinely searched for before making decisions, perception that local politicians were influenced by research evidence, percentage of retrieved articles read, expecting to use systematic overviews in the future and the perception that systematic overviews would overcome the barrier of not having enough time to review the literature at T2. Using a backward elimination procedure only one of the seven variables, perception of ongoing training in research methods, was removed from the final regression model. Diagnostic tests identified only two outlying cases having a significant impact on the regression coefficients and therefore were removed from the analysis. The results of the final regression model are presented in Table 18.

The final regression model included at least one variable from each of the four major characteristics. Two of the variables were organizational, two were individual, one was environmental and the final one was a characteristic of the innovation. The high adjusted $R^{2}$ of this model resulted in $41.0 \%$ of the variation in the influence of systematic overviews on program justification decisions being explained.

The most influential variable on the influence of the systematic overviews on program justification decisions was the percentage of retrieved articles read in the past month. The results demonstrated that as the percentage of retrieved articles read increased so did the influence of the systematic overviews on program justification decisions. The second most influential variable 
was the environmental characteristic of the perception that local politicians were influenced by research evidence. In this case the more respondents perceived politicians to be influenced by research evidence the greater the influence the systematic overviews had on program justification decisions. The third most important variable was another individual characteristic, whether the respondent expected to use the systematic overview in the future. Respondents indicating at T2 that they expected to use the overviews in the future perceived the systematic overviews as having greater influence on program justification decisions than those who had not expected to use the overviews in the future. The fourth most important variable was the extent to which respondents perceived their health units to routinely search for information before making decisions. These results demonstrated that as the search for information increased, so to did the perception that the systematic overviews influenced program justification decisions.

The final two variables in the model were the perception that systematic overviews would overcome the barrier of time at $\mathrm{T} 2$, and the perceived value the organization placed on research evidence. In terms of the systematic overviews overcoming the barrier of time at $T 2$, the results demonstrated that the greater the perception that systematic overviews would overcome this barrier, the greater the perceived influence the systematic overviews had on program justification decisions. The results for the perceived value the organization placed on research demonstrated that the more strongly respondents agreed that their organization valued research, the greater the perceived influence the systematic overviews had on program justification decisions.

Several interaction terms were tested in this analysis, which based on the literature, may have had a significant impact on the regression analysis. These included percentage of articles read*expect to use; perceived organizational value*expect to use; organizational value*overcoming time barrier $\mathrm{T} 2$; and politicians influenced by research*search for 
information. None of these interaction terms were statistically significant when assessed in the regression model. A normal probability plot of the observed and expected probabilities identified a strong linear relationship. The tolerance values ranged from .820 to .961 , which demonstrated that very little of the variation observed in each of the independent variable was explained by the other variables in the model. Therefore, multicollinearity was not a concern among the significant variables included in this analysis.

\subsection{Influence of Systematic Overviews on Program Evaluation}

\section{Results of the Analysis of Variance}

The results from the 49 analyses of variance when the dependent variable was the perceived influence of the systematic overviews on program evaluation decisions are presented in Table 19. The results demonstrated that only four of the 49 independent variables were statistically significant in explaining the variation in the influence of the systematic overviews on program evaluation decisions. The four variables included one organizational characteristic, two individual characteristics and one innovation characteristic.

The significant organizational characteristic was the number of programs the public health unit currently provided. The two individual characteristics were the percentage of articles read in the past month and the number of days spent at conferences or workshops in the past year. The significant characteristic of the innovation was the perceived relevance at $\mathrm{T} 2$ of the systematic overviews to decisions being made.

\section{Bivariate Correlation Analysis: Program Evaluation}

The Pearson correlation matrix of the four significant variables and the dependent variable is presented in Table 20. Although the bivariate analysis did not identify any unacceptably high correlation coefficients, only one of the independent variables, the perceived 
relevance of the systematic overviews at $\mathrm{T} 2$ to decisions being made, was significantly correlated with the influence of systematic overviews on program evaluation decisions. Therefore, the linear regression analysis conducted for this outcome included only one variable.

Significant Predictor of Systematic Overviews Influencing Program Evaluation Decisions: Results of the Simple Linear Regression

The final linear regression analysis was conducted using 64 cases and the one characteristic of the innovation. Diagnostic tests identified only one outlying case having a significant impact on the regression results and therefore was removed from the analysis. The results of the final model are presented in Tables 21 .

Although the final model was significant at 0.006 , the explanatory power of this regression analysis was somewhat low in comparison to the two previous multiple linear regressions for program planning and program justification. The results demonstrated that this regression model explained only $10.1 \%$ of the variation in the influence the systematic overviews had on program evaluation decisions. The standardized Beta coefficient for the perceived relevance of the systematic overviews at $\mathrm{T} 2$ to decisions being made, indicated that the more relevant respondents perceived the systematic overviews to be to decisions being made the greater the perceived influence the overviews had on program evaluation decisions. Although the linear regression for program evaluation did not produce as high an $\mathrm{R}^{2}$ as the multiple regressions for program planning and program justification, this model did identify that the perceived relevance of systematic overviews was a very strong predictor variable. 


\subsection{Influence of Systematic Overviews on Policy Development Decision}

\section{Results of the Analysis of Variance}

Seven characteristics were statistically significant in the analyses of variance, in explaining the variation in the influence of systematic overviews on policy development decisions. The results are presented in Table 22. Four of the variables were organizational characteristics, one was environmental, and the remaining two were individual characteristics.

The four significant organizational characteristics included the perceived value the organization placed on research evidence, perceived adequate financial resources, the perception of ongoing training to staff in research methods, and the perceived importance the health unit routinely placed on research evidence to make decisions. The only significant environmental characteristic was the perception that local politicians were influenced by research evidence. The two significant individual characteristics were age. and whether the participant had direct access to online database searching.

\section{Bivariate Correlation Analysis: Program Planning}

The Pearson correlation matrix is presented in Table 23. The results demonstrated that many significant associations existed between most of the variables, particularly the organizational characteristics. However, despite being significant at the $p<0.05$ and 0.01 levels, none of the correlation coefficients exceeded .437 . This was considered to be of a moderate degree of correlation, and therefore all seven variables could be used in the multiple linear regression analyses. Further examination of the correlation coefficients between the dependent variable and the independent variables illustrated that one variable, the perceived importance of research evidence in decision-making, was not significantly correlated with the outcome, and therefore was excluded from further analysis. The one environmental variable, since it was the only significant variable in this category, and was significantly correlated with the outcome and 
had a correlation coefficient above 0.2 , proceeded directly to the full multiple linear regression analysis.

Results of the Multiple Linear Regression of the Influence of Systematic Overviews on Policy Development - Organizational Characteristics

One of the three organizational variables, perceived adequate financial resources, included in this multiple linear regression analysis was removed using the backward elimination procedure. The two variables remaining in the model were the perceived value the organization placed on research evidence, and the perception of ongoing training to staff in research methods. Both variables were included in the full multiple linear regression model for policy development.

Results of the Multiple Linear Regression of the Influence of Systematic Overviews on Policy Development - Individual Characteristics

Both of the individual variables were significant in this multiple linear regression model and therefore were retained for the full regression model. The variables included age and having direct access to on-line database searching.

Significant Predictors of Systematic Overviews Influencing Policy Development: Results of the Multiple Linear Regression

The final multiple linear regression model was conducted using 82 cases and the five significant variables identified above. This included the perceived value the organization placed on research, the perception of ongoing training to staff in research methods, the perception that local politicians were influenced by research evidence, age and having direct access to on-line 
database searching. Using a backward elimination procedure, two of the five variables, the perception of ongoing training to staff in research methods, and the perception that local politicians were influenced by research evidence were removed from the model. Diagnostic tests identified three outlying cases that were removed from the analysis. The results of the final multiple regression model are presented in Table 24. The explanatory power of this model was quite high with $30.5 \%$ of the variation in the perceived influence of the systematic overviews on policy development decisions being explained by the model.

The variable exerting the greatest influence on the dependent variable was the perceived value the organization placed on research to make decisions. The results demonstrated that the more strongly respondents perceived their health unit valued research to make decisions, the greater the perceived influence the systematic overviews had on policy development decisions. The second most important variable was age category. The results indicated that older respondents perceived systematic overviews to have had greater influence on policy development decisions than younger respondents. The final variable included in the model was having direct access to on-line database searching. The results suggested that respondents who did not have access to online database searching perceived systematic overviews as having greater influence on policy development decisions than those who did have direct access to online database searching.

Interaction terms, which were based on the literature, were also tested in the model. These included terms such as organizational value*age; organizational value*on-line access; and age* on-line access. None of these interaction terms were significant. The normal probability plot of the observed and expected identified a mostly linear relationship for the observed data. An examination of the tolerance values, which ranged from .971 to .984 , demonstrated that very 
little of the variation observed in each independent variable was explained by the other variables in the model. Therefore, multicollinearity was ruled out as having a significant impact on this multiple linear regression analysis.

\subsection{Influence of Systematic Overviews on Staff Development Decisions \\ Results of the Analysis of Variance}

Only one of the 49 independent variables was statistically significant in the analyses of variance when the dependent variable was the influence of the systematic overviews on staff development decisions. The results are presented in Table 25. The one significant innovation characteristic was the perceived appropriateness of the timing for receiving the systematic overview.

\section{Bivariate Correlation Analysis: Staff Development}

The Pearson correlation coefficient, presented in Table 26, demonstrated that there was a statistically significant association between the perceived appropriateness of the timing for receiving the overviews and their perceived influence on staff development decisions. Therefore this one variable was included in a simple linear regression analysis.

\section{Significant Predictor of Systematic Overviews Influencing Staff Development: Results of the}

\section{Linear Regression}

The final linear regression model was conducted using 63 cases and the one remaining variable, the perceived appropriateness of the timing for receiving the systematic overviews. Diagnostic tests on the regression results identified two outliers that were removed from the analysis. The results of the final regression model are presented in Tables 27. Given there was only one variable included in the model the explanatory power of the model was modest. This 
linear regression model explained $14.9 \%$ of the variation in the influence of the systematic overviews on staff development decisions.

The moderately high standardized Beta coefficient demonstrated that the perceived appropriateness of the timing for receiving the systematic overviews had considerable impact on the perceived influenced of the systematic overviews on staff development decisions. The results also demonstrated that as respondents perceived the timing of the systematic overviews to be more appropriate, the greater the perceived influence the overviews had on staff development decisions. 


\subsection{Discussion}

This dissertation has presented the results of six distinct outcomes related to the utilization of five systematic overviews among public health decision-makers in Ontario. All six outcomes were measured at the level of the individual decision-maker. The theoretical underpinnings of this dissertation arose from a variety of bodies of literature including the diffusion of innovations, research dissemination and utilization, and the management literature. The Diffusion of Innovations theory played an important role in the selection and definition of key independent variables, particularly in identifying characteristics of the organization, environment, individual, and the innovation.

The results of a meta-analysis on diffusion of innovations studies conducted by Damanpour (1991) pointed out that the adoption of innovations was subject to influences by individual, organizational and environmental factors. Other researchers, as discussed throughout this dissertation, have identified factors of the innovation as playing a critical role in innovation adoption, particularly in the management literature. These four categories of variables therefore, formed the basis of the theoretical framework discussed in this dissertation. Even though there was considerable literature demonstrating the significant associations between each of these four categories of variables and innovation adoption, there has been little research to date examining these relationships in the public health setting in Ontario and Canada. Since the utilization of research evidence, and in particular, systematic overviews of the effectiveness of public health interventions, is currently a pressing issue among decision-makers in Ontario, this research was not only timely but needed for guiding future dissemination efforts in the province.

The innovation was comprised of five systematic overviews of the effectiveness of public health interventions that were disseminated to public health decision-makers in public health 
units in Ontario in 1996. The topics of the overviews were chosen in collaboration with provincial advisory groups to ensure their relevance to current policy and program decisions. At least three of the five overviews were related to health programs that had been targeted for updating and large scale resource investment by the provincial government between 1996 and the time the data was collected for this study in 1998 . These included the systematic overviews related to parent-child health, home-visiting and community-based heart health promotion. This doctoral study was a follow-up to an earlier study, funded by the Ontario Health Care Evaluation Network and conducted in 1996, that identified barriers to utilizing research evidence for public health decision-making, as well as public health decision-makers' perceptions of whether systematic overviews could overcome these barriers.

This was the first study of its kind in Ontario and Canada to assess the influence of a variety of variables on the adoption of an administrative innovation among public health decision-makers. It was also the first time data on perceptions of organizational and environmental characteristics and their influence on key public health decisions, have been collected and examined from this group of health professionals. This study was designed to address one of the major weaknesses inherent in many research utilization studies to date, which has been the failure to assess the intended audiences' perceptions of the innovation prior to receiving the innovation. Data on how the intended audience perceived the innovation prior to receiving it will help to clarify the relationships between the innovation and the independent variables. In this study data on respondents' perceptions of the usefulness of the systematic overviews. which were assessed by measuring the relative advantage, compatibility and complexity of the innovation, were collected both before and after receiving the systematic overviews in 1996. 


\subsection{Impact of the Systematic Overviews Among Decision-Makers}

\section{Research Question}

What percentage of public health decision-makers reported using the systematic overviews two years after receiving them (Objective 1).

The results of this study demonstrate that almost $63.0 \%$ of decision-makers reported using at least one of the five overviews, in some way, over the last two years. The majority of respondents indicated that the overviews were used in collaboration with other pieces of evidence to make program and policy decisions. The attainment of a $63.0 \%$ utilization rate was almost double that observed three months after the systematic overviews were disseminated in 1996. At that time, $33.0 \%$ of respondents indicated they had used at least one of the overviews in some way, since the dissemination of the overviews to them two years earlier (Ciliska et al, 1999). The rate of utilization of the systematic overviews was consistent across the province with at least one person from each health unit stating they had used a systematic overview over the past two years. The results for the outcome "overall use" of the systematic overviews have been summarized in Tables 28-30, and will be referred to throughout this discussion. Similar findings have been reported among staff nurses in British Columbia where $77.0 \%$ of a random sample indicated they had used research findings at least sometimes in their practice (Varcoe et al., 1995) 


\section{Research Question \#2}

Were there significant differences in overall use of the systematic overviews according to the position the decision-maker held at the time the overviews were disseminated. For example did program managers and program directors use the systematic overviews significantly more than Medical or Associate Medical Oficers of Health. (Objective 2)

The results in Table 28 demonstrated that the position the study respondents had at the time when the systematic overviews were disseminated was a significant factor in whether the overviews were used during the two year follow-up period. The results not only identified position as a significant predictor of overall use of the overviews, but also demonstrated that program directors and program managers were significantly more likely to have used the systematic overviews than were medical and associate medical officers of health. The results also demonstrated that program directors were slightly more likely to have used the systematic overviews as compared to program managers, but this difference was not statistically significant. These findings are corroborated by Lomas, (1990) who suggested that the type of information provided to decision-makers must vary with the functions of the various levels of decisionmakers. Lomas was suggesting here that decision-makers at the program manager level might have different information needs than program directors, medical officers of health, and policy makers. Therefore, systematic overviews might fill the research evidence needs of program managers to a greater extent than for program directors and medical/associate medical officers of health.

These results were important because they identified who the target audience should be for systematic overviews of the effectiveness of public health interventions. The systematic overviews that were disseminated to these public health decision-makers were large, detailed documents that made recommendations for specific public health interventions. These are the types of decisions that are currently made by program managers and program directors in 
Ontario (Ontario Ministry of Health, 1989), which are then passed along to the medical officer of health and the local board of health for final approval. The results make sense then in that those decision-makers most likely to use these systematic overviews should be program managers and program directors who are responsible for making decisions about specific interventions. Medical officers of health may not find these overviews as useful, since the decisions they are involved in are at the level of deciding which programs should be provided and resource allocation as opposed to which specific interventions within programs should be provided.

Therefore these results raised the question of what type of research evidence would be most useful for medical/associate medical officers of health. It may be that short, summary statements of one or two pages in length, that clearly outline the overall findings of the overviews, such as 'do the interventions have the desired effect and for whom', could be the most applicable format of research evidence for medical/associate medical officers of health. However, this type of research evidence would not be as useful to program managers and directors since they must make decisions regarding specific interventions to include in the operational plans. Further exploration of this issue is needed before definite conclusions can be made.

Table 29 demonstrates that the participant's position was only a significant predictor variable when the outcome was overall use of the systematic overviews. It is interesting to note that the participant's position was not a significant factor in predicting the influence of the systematic overviews on public health decisions related to program planning, program justification, program evaluation, policy development and staff development. 


\section{Research Question \#3}

Which characteristics, such as organizational, environmental, individual and characteristics of the innovation were most strongly associated with utilization of the systematic overviews. (Objective 2)

Although the combination of characteristics included in the final regression model for all six outcomes were different for all but one of the outcomes, the characteristics exerting the greatest magnitude of effect on the dependent variables, were either innovation or organizational characteristics. Even for the one outcome that did not have an organizational or innovation characteristic with the greatest magnitude of effect, more than half of the variables included in that final model were either organizational or innovation characteristics. A summary of these results is presented in Tables 28 and 29.

These results suggest that characteristics of the innovation were the most significant variables associated with all six of the outcomes examined in this study. For example a characteristic of the innovation had the greatest magnitude of effect on four of the six outcome variables. including overall use of the overviews, and the perceived influence the systematic overviews had on decisions related to program planning, program evaluation and staff development. In fact, for two of these four outcomes, program evaluation and staff development decisions, a characteristic of the innovation was the only variable included in the final model. Similar findings to these were reported almost thirty years ago when Utterback (1971) illustrated that measures of the perceived relative advantage of an innovation were the primary determinants of its adoption in an industrial market. Although the setting for that study was markedly different than the one in this dissertation, the similarity in findings demonstrates the overall significance of characteristics of the innovation to innovation adoption, among a wide variety of users and settings. 
Overall. organizational characteristics were shown to play an important role in facilitating the utilization of systematic overviews among public health decision-makers. For example, an organizational characteristic had the largest magnitude of effect on one outcome, policy development decisions. In addition, organizational characteristics were significant predictor variables for two other outcomes, the perceived influence of the systematic overviews on program planning and program justification decisions. Therefore, among public health decisionmakers, organizational characteristics were important factors in facilitating the utilization of systematic overviews. It is also important to point out that some of the organizational characteristics measured in this study had very limited variability, which may have resulted in these characteristics being insignificant in the analysis. For example, approximately $95 \%$ of respondents indicated that their health unit strictly adhered to policies and procedures. Since this left little room for variability among responses, it was not likely that a significant association between this characteristic and the dependent variable would be found. These findings suggest that this characteristic might not be an appropriate measure for predicting research utilization, or that an alternative way of measuring this characteristic, that produced greater variability in responses, was needed.

An interesting finding in this study was the observed importance of individual characteristics. For example an individual characteristic had the greatest magnitude of effect on one outcome, the influence of the systematic overviews on program justification decisions, and other individual characteristics were shown to be significant predictor variables for three other outcomes, overall use, and the influence of the overviews on program planning and policy development decisions. These findings were surprising given previous research results reporting 
that individual factors were not significantly associated with research utilization (Ciliska et al., 1999; Kaluzny et al., 1973; Kaluzny et al., 1974).

In summary, the results demonstrated that characteristics of the innovation had the greatest impact on utilization of the systematic overviews. This is conclusion is based on examining which category of characteristics were most often associated with the outcome variables as well as which category of characteristics had the greatest magnitude effect on the outcomes. This was followed by organizational characteristics and then individual characteristics. Finally these results demonstrated that characteristics of the environment had the least amount of influence on the utilization of the systematic overviews.

There was yet another way to examine the results of the influence that each independent variable had on the utilization of the systematic overviews, which has been summarized in Table 30. There were a total of eighteen variables included in the six, final multiple logistic and linear regression analyses, although some variables were included in more than one model. Summarized in this way, the results demonstrated that characteristics of the individual were included more often than either innovation or organizational characteristics in the final regression models. For example, characteristics of the individual comprised seven of the eighteen variables included in all the models, while characteristics of the innovation were included six times, and organizational variables only four times. Therefore, the results indicated that although the characteristics of the innovation were more strongly associated with the six outcome variables than any other type of characteristic, individual characteristics also played an important role in most of the outcomes.

No matter how the results are summarized however, the overwhelming importance of the characteristics of the innovation cannot be missed. They were not only important for predicting 
overall use of the systematic overviews, but also for predicting the influence of the overviews on all but one of the decisions examined in this study. The important aspect to consider is that characteristics of the innovation have not been routinely included in research utilization studies in health care settings, or in the development of dissemination strategies. However similar results have been reported by Meyer et al., (1988) who studied the adoption of an innovation among 25 private, non-profit hospitals in a large midwestern US city. Meyer (1988) reported that characteristics of the innovation explained most of the observed variation in innovation adoption, even when other characteristics such as organizational and environmental variables were included in the regression model. These results suggest that in order to fully understand the research utilization process in the field of public health and possibly health care in general, greater emphasis must be placed on measuring and understanding the impact of individual's perceptions of the innovation on research utilization. These results also demonstrated that characteristics of the innovation could be important factors that warrant inclusion in future dissemination strategies. This means that dissemination strategies need to consider either how to change the intended users' perceptions of the innovations to a position that will facilitate utilization of systematic overviews, or change the innovation itself to become more timely and easy to use, to name but a few options. Although at this point it is unclear if dissemination strategies that incorporate activities aimed at changing perceptions of the innovation would be useful in promoting research utilization, there was enough evidence produced in this study to warrant further investigation in this area.

Generally, the associations between the independent variables and the outcomes were supported in the diffusion of innovations and research dissemination and utilization literature. For example, the more strongly respondents perceived systematic overviews to overcome the 
barrier of limited critical appraisal skills at $\mathrm{T} 2$, the more likely they were to have used a systematic overview since its dissemination. A similar result was observed by Royle et al, (1997), who reported that $62.0 \%$ of directors of nursing believed that courses on critical appraisal would be necessary to facilitate research utilization. Also, the greater the perceived relevance of the systematic overviews at $T 2$ to decisions being made, the greater the perceived influence of the overviews on both program planning and program evaluation decisions. A number of research utilization studies have supported this finding by demonstrating that the relevance of the innovation and its ease of use are positively associated with utilization (Bigoness et al., 1981; Closs et al.. 1994; Funk et al., 1995; Hunt, 1996). Other researchers have suggested that research evidence should not only be actively disseminated to the intended audience but must be accompanied by a step by step package suggesting how the findings should and could be used in the practice setting (Gustafson et al., 1995; Horsley et al., 1983).

The same type of pattern held true for the organizational and even the one significant environmental characteristic. For example, the greater the perception that the organization valued the use of research, the greater the perceived influence of the systematic overviews on program planning, program justification and policy development decisions. This finding has been supported in the research utilization literature which suggested that the greater the perceived support for research evidence from the organization the greater its utilization (Butler, 1995; Champion et al., 1989; Crane, 1995; Pettengill et al., 1994; Swanson et al., 1992; Varcoe et al., 1995; White et al., 1995). In terms of environmental characteristics, the greater the perception that local politicians were influenced by research, the greater the perceived influence of the systematic overviews on program justification decisions. 
Given the lack of research evidence related to dissemination and utilization among public health decision-makers, and conflicting results from the literature that does exist, it was unclear what direction to expect in the associations between the individual characteristics and the dependent variables. Despite this lack of empirical rationale, the relationships between two of the individual characteristics and one of the outcomes were in a direction that was opposite to what one might intuitively expect. For example, age was positively associated with the influence the systematic overviews had on policy development decisions meaning as age increased, so did the perceived influence of the systematic overviews on these decisions. Although at least one study has reported a similar relationship between age and innovation adoption among a random sample of primary care physicians (Freiman, 1985), this result is contrary to what has been reported in the majority of studies. Research findings have suggested that younger decision-makers, with more recent undergraduate and postgraduate experiences, are more likely to use research evidence (Damanpour, 1991). In addition younger age has also been shown to be associated with increased knowledge of and comfort with critical appraisal and evidence-based practice (Dalrymple, 1990; Davis et al., 1997). However, the results presented in this study indicated the opposite in terms of the perceived utilization of the systematic overviews for policy development decisions. One explanation for this might be that the older respondents in this study were in positions to make policy development decisions whereas the younger decision-makers were not.

The second individual variable whose association was in the opposite direction from that which might be intuitively expected was having direct access to on-line database searching. The results demonstrated that respondents who did not have access to on-line database searching, perceived the influence of the systematic overviews on policy development decisions to be greater than those who had direct on-line access to database searching. Although intuitively this 
seems in opposition to what might be expected, there is a reasonable explanation for this finding. It might have been possible that those respondents without on-line access to database searching may have routinely had much less research evidence to base policy development decisions upon than those who did have on-line access to databases. Therefore, those with on-line access may have had considerably more research evidence to review, one piece of which was the systematic overviews, than those without access to on-line database searches. Therefore, having a large amount of literature available might have reduced the influence the overviews had policy development decisions for respondents who had direct access to on-line database searching.

Although some generalizations can be made about the importance of the types of characteristics on the outcome variables, the results were much less conclusive when specific independent variables were examined. In fact, the results demonstrated that there was only one variable that had the greatest magnitude of effect for more than one outcome. The independent variable, the perception that the systematic overviews were relevant to decisions being made, was the only variable having the greatest magnitude of effect in the regression models for more than one outcome. This demonstrated that none of the independent variables consistently influenced all or most of the outcomes. For example, the significant variables that predicted overall use of the systematic overviews were all different from those identified as important for predicting the influence the overviews had on program planning decisions. Therefore, the results clearly demonstrated that different combinations of variables were significantly associated with each of the six outcomes. One question arising from these results is whether different dissemination strategies are needed when different types of utilization are desired.

These findings also pointed out the importance of presenting research evidence in easily understood, relevant and easy to follow ways to decision-makers. Those respondents who did not 
perceive the overviews to be easy to use were almost half as likely to have used the overviews as those who thought they were easy to use. Therefore, this suggested that the presentation of research evidence played an important role in its utilization. This finding was supported by Closs \& Cheater (1994), and MacPhail (1997), who suggested that researchers should finds ways of presenting their findings in a clear, accessible and easily understood form for the intended audience to use. Also, the results demonstrated that as systematic overviews are being prepared for dissemination efforts should be taken to ensure that they 1) reflect the needs of program managers and program directors, 2) are presented in ways that are perceived as easy to use for this specific population, and 3) involve a marketing approach demonstrating that systematic overviews will overcome the barrier of limited critical appraisal skills.

The results of this study demonstrate the importance of the value the organization placed on research and the amount of routine reading reported among decision-makers. These results suggest that the greater the perception that the organization valued research, the greater the perceived influence of the systematic overviews on program planning, program justification and policy development decisions. This indicates that future dissemination strategies must first address. and change if needed, public health units' perceptions of the value of research for decision-making. These results also demonstrate that dissemination strategies should focus on encouraging decision-makers to read research evidence on a routine basis. This finding has been supported in other research utilization studies where nurses indicated that more time to read research during work time was needed to facilitate its use in practice (Pettengill et al., 1994; Stolk et al., 1995).

Despite the need for more research to identify additional factors associated with influencing decisions related to program evaluation and staff development, these were important 
findings because they identified two key factors that can be used in the short term, to facilitate systematic overview use in these areas. The significant variable from each model, the perceived relevance of the systematic overviews to decisions being made, and the perceived appropriateness of the timing for receiving the overviews, suggested that perceived relevance and timing are important factors that should be incorporated into future dissemination strategies.

For the outcome, the influence of the systematic overviews on policy development decisions, an organizational characteristic had the greatest magnitude of effect when compared to the two individual characteristics included in the final regression model. However, none of the innovation characteristics were significantly associated with influencing policy development decisions. For this outcome as the perception that the value the organization placed on research evidence increased, so did the perceived influence of the systematic overviews on these policy decisions. This is important, because again it provides direction in the development of future dissemination strategies, to promote an organizational culture that values research evidence.

The two remaining significant individual characteristics in this regression model for policy development decisions also provided some important insight into this outcome. Table 28 demonstrated that age and direct access to on-line database searching were significantly associated with greater perceived influence of the systematic overviews on policy development decisions. One possible explanation for having both variables in the same model, and both occurring in directions opposite to what might be expected, could be that the older respondents were also the same respondents who did not have direct access to on-line database searching. If this hypothesis was correct this would provide another explanation for why older respondents perceived the overviews to have greater influence on policy development decisions than younger ones. It might be that older respondents are not only more likely to be in positions to make policy 
deveiopment decisions, but also are less likely to have access to on-line database searching, and therefore have less research evidence with which to base decisions upon. These results have actually produced more questions than answers at this point, with more investigation, particularly face-to-face interviews, needed to understand more clearly the relationship between age, access to on-line database searching and the influence of the systematic overviews on policy development decisions. Other questions raised by these results could focus on identifying who the younger respondents were, whether they had been involved in policy development decisions, and for those that were, what evidence they have used in the past to make these types of decisions.

There was only one outcome, the influence of the systematic overviews on program justification decisions, which produced somewhat unique results when compared to the other outcomes. with respect to the order of the variables with the greatest magnitude of effect. The two variables with the greatest magnitude of effect on this outcome were the individual characteristic, percentage of articles read in the past month, and the environmental characteristic, the perception that local politicians were influenced by research evidence. Although there were a total of six significant variables in the final regression model, the two organizational characteristics were the fourth and sixth variables in the model, and the innovation characteristic was the fifth variable included in the model.

These results suggested that there might have been something different about program justification decisions as compared to all the other decisions. For example, the importance of the individual characteristics, being the first and third variables included in the model, suggested that program justification decisions may be more of a program manager decision as opposed to more organizationally based decisions such as program planning and policy development. These 
results could be interpreted in two ways. The first interpretation suggests that as the percentage of retrieved articles read per month increased, so did the perception that systematic overviews influenced program justification decisions. However, another interpretation might be that respondents who read a lot also supported systematic overviews, and therefore perceived the influence of the overviews on program justification decisions to be greater. Although each interpretation has different implications for the development of dissemination strategies, the basic message suggests that routine reading of research evidence is positively associated with the utilization of the systematic overviews. In general, the reading of research literature has been shown to be a significant predictor of research utilization among nurses working in community hospitals (Coyle et al., 1990).

Another important individual characteristic associated with the influence of the systematic overviews on program justification decisions was expecting to use the systematic overviews in the future. The results indicated that if respondents identified a need for the overviews in the future, then public health decision-makers were more likely to use the overviews in the future. This was an important finding because it pointed out that if decisionmakers come across evidence they identify as being useful to them in the future, they are more likely to use that research in the future.

The second most important variable in this model was the perception that local politicians were influenced by research evidence. The results demonstrated that the greater the perception that politicians were influenced by research evidence the greater the perceived influence the systematic overviews had on program justification decisions. This finding was somewhat expecied given the recent downloading of public health services to the municipal level in January. 1998. As a result of the downloading local politicians have become much more 
involved in decision-making regarding the allocation of resources and service provision with respect to public health services. Given the limited resources available at the municipal level for public health services, many health units and program managers have been faced with justifying to local politicians the need for ongoing funding for many public health programs. Therefore these findings indicated, to some extent, the relationship that has emerged between public health decision-makers and local politicians with respect to the types of evidence that have been useful in convincing local politicians to continue funding specific public health programs. Although it would be difficult to investigate, given that the downloading of public health services has already occurred, it would be interesting to determine if the perception that politicians were influenced by research evidence was as significant a variable before the downloading occurred.

The three remaining variables in the final regression model on program justification decisions included two organizational characteristics and one innovation characteristic. The two organizational characteristics were the perceived value the organization placed on research and the perceived extent to which the organization routinely searched for information before making a decision. The two organizational variables seemed intuitively related in that the more organizations valued research evidence, the more likely they might be to search for research evidence before making decisions. However, it is interesting to note that although routinely searching for research evidence before making decisions significantly predicted the influence of the systematic overviews on program justification decisions, the perceived importance of research evidence in the decision-making process was not a significant predictor of this outcome. This is a conflicting result because it suggests that public health decisions-makers appreciate the value of searching for research evidence to be used in decision-making, but then fail to incorporate this evidence into the decision-making process. These findings suggest that closer 
examination of the decision-making process with respect to the utilization of systematic overviews and research evidence in general among public health decision-makers is necessary. It is also likely tnat the most appropriate design for examining these issues more closely is a qualitative study that incorporates face-to-face interviews, focus group discussions, and participant observations.

The innovation characteristic, the perception that the systematic overviews would overcome the barrier of not having enough time to review the literature at T2, also seemed to be closely tied with the two organizational variables discussed above. For example, it might be possible that the greater the perception that systematic overviews would overcome the barrier of time, the more organizations might begin to value research and then the more likely systematic overviews might be searched for before making decisions. In addition, if a positive experience occurs during the use of the overviews, this again may facilitate greater organizational value being placed on using this type of evidence on a more consistent basis.

These results demonstrate that program justification decisions might be inherently different from the other public health decisions. since the level of decision-making is at the program manager level as opposed to the medical officer of health. This would explain to some degree why this was the only outcome for which individual and environmental characteristics were more strongly associated with the influence of the systematic overviews on these decisions than organizational and innovation characteristics.

The summary of the results presented in Table 30, illustrates the overall importance of all 13 significant independent variables. It demonstrates that one of the keys to facilitating utilization of the systematic overviews in the future might be to promote their value at the organizational level and in illustrating how the use of systematic overviews will benefit 
individual health units and their respective populations. Similar findings were also reported by Stolk and Mayo (1995). It also clearly points out the need to produce systematic overviews that are perceived as relevant to the intended audience and are relevant to decisions that are being made. One way to obtain these perceptions could be from focus group discussions, as was demonstrated from the information obtained from the focus group discussions in the OHCEN study. The importance of promoting the routine reading of research evidence was also clearly identified in this dissertation, along with a number of suggestions of how this might be achieved. Finally, the results indicate that if systematic overviews are perceived as relevant to upcoming decisions, and public health decision-makers expect to use the overviews in the future, then the overviews were more likely to be used.

The results of this dissertation demonstrate that among public health decision-makers, individual characteristics appear to be much more important than environmental characteristics and to some extent, are as important as the organizational characteristics. In addition, only one environmental characteristic was significantly associated with one outcome, which was the influence the systematic overviews had on program justification decisions. These results were somewhat surprising given the downloading of public health services to the municipal level in 1998, which occurred six months prior to the collection of data for this dissertation. However, it might be that not many decisions concerning public health services at the municipal level had been made by that time by local city/regional councils. Another explanation may be that of all the decisions made in public health, only those related to program justification require the approval of local politicians, thereby resulting in the importance of this variable only for these decisions. It might be that decisions related to program planning, program evaluation, local public health policy development and staff development fall within the jurisdiction of the 
medical officer of health and the management team within health units, and do not require approval from the local city/regional councils. Therefore, at this point, it would appear that environmental factors played a rather minor role in influencing the use of systematic overviews for public health decisions, except for those associated with program justification.

\section{Research Question $\# 4$}

Which types of public health decisions, (program planning, program justification, program planning, policy development, and staff development) will systematic overviews infuence the most (Objective 3).

Among individual decision-makers the two multiple linear regression models with the most explanatory power were for the influence of the systematic overviews on program justification decisions and the influence of the overviews on policy development decisions. Forty-one percent of the variation in the influence of the systematic overviews on program justification decisions was explained by the predictor variables included in the model. These results demonstrated the importance individual and environmental characteristics had in influencing program justification decisions, as well as illustrated how both organizational and innovation characteristics played a secondary role in the utilization of systematic overviews for these types of decisions. The high adjusted $R^{2}$ attained in this regression model provided considerable confidence that the identified variables were integral to influencing program justification decisions, and were helpful in identifying potentially effective dissemination strategies which will be addressed later in this chapter.

The model with the second highest explanatory power was related to the influence of the systematic overviews on policy development decisions. For this outcome the three variable model explained $30.5 \%$ of the variation. These results demonstrated that a very different 
combination of variables were significantly associated with these two outcomes despite the common thread of the importance of the perceived value the organization placed on research. Again the moderately high total explained variance value for this outcome provided considerable confidence that these variables had an important impact on this outcome and also allowed for additional recommendations to be made regarding future dissemination strategies.

Very close behind the policy development outcome came the influence of the systematic overviews on program planning decisions. The explanatory power of this regression model was only $4.1 \%$ below that obtained for the policy development regression, and explained $26.4 \%$ of the variation. Three variables significantly predicted whether systematic overviews influenced program planning decisions. This moderately high total explained variation value also provided confidence that this combination of variables was associated with systematic overviews influencing these decisions.

There were two outcomes examined in this study for which little new evidence was learned. These included the influence of the systematic overviews on program evaluation decisions and the influence of the systematic overviews on staff development decisions. Each regression model identified only one significant predictor variable and the total explained variance for each model was low in comparison to the other three multiple linear regression analyses. However, these findings were important because they supported the overall result from this study that characteristics of the innovation were the most important factors associated with utilization of the systematic overviews. However, these results were also disappointing because many questions remain unanswered about how to facilitate greater influence of systematic overviews en program evaluation and staff development decisions. 
There are several reasons why the results for these two outcomes might have occurred. First, it might be that systematic overviews simply are not relevant to decisions related to program evaluation and staff development. Second, it might be that decision-makers have not previously used systematic overviews to make these types of decisions and therefore are not aware they can be used for such decisions. Third, they cannot be easily interpreted for use in program evaluation and staff development decisions. Whatever the reason, there is still reason to continue investigating how or when systematic overviews can be used to influence these decisions.

The main reason for not dismissing these two outcomes immediately comes from the observation of focus group discussions with senior public health decision-makers that were conducted for the OCHEN study in 1996. During these focus group discussions, participants who were all familiar with systematic overviews, were asked to name the types of public health decisions they believed could and should be influenced by systematic overviews. Analysis of the responses identified the five decision areas that have been reported on in this dissertation. Along with program planning, program justification and policy development, these decision-makers also believed that systematic overviews could and should play a role in program evaluation and staff' development decisions.

These results raise the question of how systematic overviews can be used to influence these types of decisions. One scenario could be that the results of a systematic overview suggest that home visiting to families at risk for abusing their children are effectively treated by both public health nurses and peer home visitors. If a health unit is using only public health nurses for these interventions, this systematic overview might influence a decision to evaluate the effectiveness of using a combination of both service providers or comparing one to the other. In 
terms of staff development decisions, a systematic overview might suggest that communitybased interventions directed at promoting heart health need to be multi-faceted and multisectoral, in order to be effective. These results might influence a decision to provide training to staff in community-based activities. These are only two scenarios for which the use of systematic overviews for program evaluation and staff development decision-making would be appropriate. Therefore, it would be worthwhile to examine these two outcomes further before ruling them out as important outcomes.

\subsection{Implications for Facilitating the Utilization of Systematic Overviews}

Although many important findings emerged from this study a number of new questions were also raised. One of the reasons for identifying variables that predict the utilization of these systematic overviews was to gain a better understanding of why and how they were being used. Another important reason was to use the knowledge gained from this study, as well as the dissemination literature, to develop dissemination strategies that would be effective for public health decision-makers in Ontario in the future.

The literature indicates that some dissemination strategies have been proven to be effective in changing medical practice to some extent. However, although some positive findings have been identified in the dissemination literature, the majority of studies have experienced only limited success (Thomson et al., 1997; Davis et al., 1992; Davis et al., 1995; Lomas, 1991; Lomas, 1993a; Oxman et al., 1995) What is also unclear, is whether strategies that are effective in one setting with certain health care professionals are as effective in other health care settings, with different health professionals. A review of the dissemination literature, which has focused primarily on physicians thus far, has identified three levels or categories of dissemination strategies with respect to their effectiveness in changing physician practice. 
The first category is comprised of those dissemination strategies that have been found to produce significant, positive change, which includes reminder systems, academic detailing and multiple interventions implemented simultaneously. The second category includes strategies that have been found to be moderately effective and includes opinion leaders and audit and feedback directed at individual physicians. The third category and least effective strategies include didactic lecture-based continuing education, and mailed, unsolicited materials (Davis et al., 1997: Dobbins et al., 1998; Sibbald et al., 1997).

Although these dissemination strategies may initially appear feasible within the public health setting and among public heaith decision-makers, when they are examined more closely it becomes more difficult to envision how academic detailing, reminder systems, and audit and feedback strategies could be operationalized consistently across the province. The following example will demonstrate the complexities of implementing an audit and feedback strategy. Public health decision-makers could be asked to choose one intervention to review for which a Best Practice Guideline has been developed. The Ontario Ministry of Health has been developing Best Practice Guidelines for public health practice based on research evidence and provincially identified needs. Each health unit would then be audited to determine how closely their practice resembled what was suggested in the Best Practice Guideline. Each health unit would then receive feedback on how their intervention compared to the Best Practice Guideline and provided a systematic overview that they could use to alter their practice so as to resemble the Best Practice Guideline more closely. Initially, this strategy appears as though it would be useful, effective and appropriate. However, closer examination of each health unit might illustrate that local population differences and needs resulted in the development of an intervention that was somewhat different from that recommended in the Best Practice Guideline. Although it was 
different from the Best Practice Guideline it might still be appropriate for that population. Therefore, if the outcome of success for this strategy was a change in practice to resemble the Best Practice Guideline, then the strategy would be shown to be ineffective, when in fact the outcome measure was inappropriate for the situation. Therefore, considerable thought must be given not only to the identification of effective dissemination strategies but also to the identification of realistic and reasonable measures of success of the strategies. The findings from the dissemination literature can be used to guide the future development of dissemination strategies in public health, but must be adapted to meet the specific needs of these decisionsmakers, which have been identified in this study.

The approach proposed in this dissertation for developing dissemination strategies for public health decision-makers in Ontario, involves building strategies around those variables identified as being important in this dissertation. For example, the value the organization placed on research evidence was a variable that was shown to be significantly associated with four of the six utilization outcomes examined in this dissertation. This finding has also been reported in numerous research utilization studies showing that increasing administrative support for utilizing research evidence in program decision-making was positively associated with its use (Funk et al., 199lb). Therefore, it seems reasonable that an effective dissemination strategy would include strategies that were directed at facilitating public health units to value research evidence and systematic overviews. One way to promote organizations to value systematic overviews might be to demonstrate clearly and specifically to public health decision-makers, the positive outcomes they should expect to experience if they utilize the overviews. These outcomes will likely vary by the topic of the systematic overviews, and by the intended decision-maker. Another action that might encourage decision-makers within public health units to value research would be to 
make research utilization an expected job responsibility. This method has been supported by a number of research utilization studies that have suggested that research utilization should be included in job descriptions and as part of the annual performance evaluation process (Armitage, 1990; Funk et al., 1995).

A comprehensive dissemination strategy should not only focus on promoting utilization of systematic overviews at the individual level but also at the organizational level. However, a precursor to developing dissemination strategies should include exploration of how organizations come to form perceptions of innovations and particularly innovations such as systematic overviews and research evidence, as well as determine how these perceptions can be changed. One way to assess these perceptions would be to use outreach visits to public health decisionmakers by members of the Public Health Effectiveness Project, who have been involved in conducting the systematic overviews. Outreach visits have been defined as the use of a trained person to meet with the target audience in their practice setting to provide information with the intent of changing the provider's performance (Thomson et al., 1997). During this visit issues related to perceptions of the usefulness of the systematic overviews could be discussed, and evidence provided demonstrating positive outcomes that have been found to be associated with their use. This type of dissemination strategy has been shown to be effective in significantly changing physician prescribing patterns (Soumerai et al., 1989; Thomson et al., 1997). Although the change being sought here is a change in perception, there is sufficient evidence to support using this dissemination strategy among public health decision-makers.

This type of approach represents a markedly different approach to research dissemination than has occurred in the past, where the emphasis has been on developing effective ways of getting the research evidence to the intended user. The approach being proposed at this time may 
be more comprehensive because it not only includes methods for distributing systematic overviews to the intended audience, but also includes the organizational, individual and environmental factors that have been shown to be associated with utilization. This method for developing dissemination strategies is supported by the work of Closs and Cheater (1994), who suggested that researchers should focus on identifying barriers to research utilization in specific areas, which would provide a basis from which local utilization strategies could be developed.

The most important contribution of this study to the literature in public health is the finding that perceptions of the characteristics of the innovation played the most crucial role in predicting utilization of the systematic overviews. However, at this time in public health, little is known about how perceptions of the innovation are formed. how they can be changed or how research evidence innovations can be altered to better meet the needs of the intended audience. Despite these shortcomings, these results demonstrate that future dissemination strategies should incorporate activities that will promote positive perceptions related to systematic overviews and potentially even research evidence in general.

The results also demonstrate that efforts should be focused on promoting positive perceptions of innovation characteristics such as the relevance of the systematic overviews to decisions being made, the appropriateness of the timing for receiving the overviews, overcoming barriers such as not having enough time to review the literature and limited critical appraisal skills, and developing systematic overviews which are easy to use. Once these basic dissemination strategies have been implemented, additional activities can be added that take into account the significant organizational, individual and environmental characteristics identified in this dissertation. 
The dissemination literature and the findings from this dissertation suggest that a comprehensive dissemination strategy incorporates an approach that is directed not only at individuals but also at the organizations within which decision-makers work, and to some extent, the environments they are located within. The dissemination strategies discussed in this dissertation address the specific needs identified by public health decision-makers in Ontario. Tailoring research evidence to the needs of the intended audience has been suggested by a number of dissemination researchers. In addition, some researchers have strongly suggested that the method of disseminating research evidence must also be tailored to the needs of the intended audience (Sibbald et al., 1997). Taking this one step further, one could develop strategies that would require researchers to include in published reports the implications of their findings for clinical practice, and to take responsibility for presenting research reports in a format that can be used in the practice setting. This same recommendation has been made by several dissemination and utilization researchers over the last 20 years (Bero et al., 1997; Brown, 1995; Crane, 1995; Hunt. 1981: Pranulis et al., 1990). Although many would argue that such activities are not within the realm of academic responsibilities, it raises the idea of an intermediary role of a research transfer specialist, who would then work with the intended audience to translate the research findings into usable practice guidelines. This same recommendation was first made more than thirty years ago and has been repeated on numerous occasions since (Halper, 1966; Lomas, 1997: Soumerai et al., 1990; Titler et al., 1994).

One of the important findings from this study was that different dissemination strategies might be necessary to produce different utilization outcomes. For instance, since none of the six outcome variables had the same combination of significant predictor variables included in the final model, it might be possible that one general dissemination strategy will not be effective in 
promoting utilization of the systematic overviews for all six outcomes. It may be necessary to develop different strategies depending on whether the desired outcome is systematic overviews influencing program planning or program justification decisions or overall use of the overviews.

The finding that overall use of the systematic overviews was significantly different according to the position the participant held when the overviews were disseminated, provided the first important piece of evidence in developing a new dissemination strategy. For example, the finding that program managers and program directors were significantly more likely to use systematic overviews than medical/associate medical officers of health, suggested that systematic overviews, in their current form as long detailed documents, are most relevant and appropriate for program managers and directors. These results raise the question of whether short. executive summary statements, clearly outlining the overall effectiveness of public health interventions, and concise recommendations for practice might be the more appropriate format of research evidence for medical/associate medical officers of health. The use of short executive summaries has recently been recommended by Bero and Jadad (1997), who used case studies to determine the research evidence needs of policy decision-makers in the health care field. Other research has illustrated that focusing on a small number of important messages is significantly associated with retention as well as utilization of information (Soumerai et al., 1990). Therefore, another dissemination strategy for public health decision-makers would be to determine who the intended target audience is, and to disseminate systematic overviews to those who are most likely to benefit from using the full, detailed document. In addition various formats of the results of the systematic overviews should be developed that address the varying needs of medical/associate medical officers of health, program managers and program directors. 
Another major finding of this study illustrates the importance of the perception that systematic overviews are relevant to decisions that are being made or will be made in the future. These findings suggest that the dissemination strategy should involve consultation with the target audience to determine which research evidence is most needed and relevant to provide input into current and upcoming decisions. This suggestion has been corroborated by a number of researchers who suggested greater communication between researchers and decision-makers was necessary in order to promote utilization (Allery et al., 1997; Bero et al., 1997; Funk et al., 1995; Gennaro. 1994; Gustafson et al., 1995; Lomas, 1997; McWilliam et al., 1997; Soumerai et al., 1990; Titler et al., 1994). As well, attention should be paid to the different types of decisions which face public health decision-makers, particularly differences between program managers, program directors and medical/associate medical officers of health. It might be that decisions at these different levels vary in terms of topic and scope, and therefore needs for research evidence might vary accordingly. Another dissemination strategy would be to consult with all levels of public health decision-makers to identify current and upcoming decisions for which systematic overviews were required.

A respondent's perception of the value the organization placed on research evidence has emerged from this study as a very important factor in the utilization of systematic overviews, among public health decision-makers. The dissemination strategy therefore, should focus on promoting positive organizational attitudes toward systematic overviews specifically and research evidence generally. To produce such change, efforts might also be needed to change the organizational culture to one that promotes the use of systematic overviews for decision-making. However, although these factors have been raised as important predictors of utilization, little is known at this time about how to go about promoting these changes. One hypothesis that could be 
tested would be to determine if dissemination strategies that focused on demonstrating the value of systematic overviews to public health units was effective in facilitating research utilization. This might include illustrating how the use of systematic overviews was effective in securing ongoing funding for programs that were in jeopardy of being eliminated. Other strategies could focus on promoting an organizational culture that encouraged the routine reading of the research literature. This might require some changes in work responsibilities and performance expectations by decision-makers, which might then foster an environment that valued research evidence. This recommendation is supported by a number of research utilization studies which have suggested that organizations interested in promoting research utilization should create a climate in which research use is an expected, valued and rewarded activity (Closs et al., 1994; Cronenwett, 1995; Funk et al.. 1995; MacPhail, 1997; McSkimming, 1996; Titler et al., 1994). Therefore, another dissemination strategy would be to develop methods to try to understand why one organizational culture values systematic overviews while another does not, and why some cultures lead to decision-makers who routinely read the research literature and others do not. These types of questions will require focus group discussions and one to one interviews with the target audience in order to understand these organizational processes.

Critical appraisal skills were also identified in this dissertation as an important barrier that predicted the overall use of the systematic overviews. This finding has been supported by a number of research utilization studies (Ciliska et al., 1999; Funk et al., 1995) that indicated that strategies to improve critical appraisal skills among health care decision-makers were associated with increased research utilization. Therefore, a comprehensive dissemination strategy should also incorporate various educational techniques to assist public health decision-makers to become more familiar with critical appraisal skills and confident in applying them to the public 
health literature. This suggestion is supported by Royle et al (1997), who reported that $62.0 \%$ of a sample of directors of nursing from community hospitals, believed that courses in critical appraisal were necessary to facilitate research utilization. There are a number of programs that currently exist, such as McMaster University's Evidence-Based Health Care workshop, as well as courses on critical appraisal that have been developed across the province that could assist decision-makers to develop these skills. This recommendation has been supported by numerous systematic overviews that examined the effectiveness of continuing medical education on changing physician and nursing practice. Continuing medical/nursing education has been defined as any educational activity or program intended to improve performance. These systematic overviews found that continuing medical/nursing education was moderately effective in changing physician and nursing practice (Davis et al., 1992; Davis et al., 1995; Lomas et al., 1988: Oxman et al., 1995; Soumerai et al., 1989; Waddell, 1991). In addition the results of one systematic overview examining the effectiveness of teaching critical appraisal skills using continuing medical education approaches, reported a positive effect on improving these skills from baseline (Norman et al., 1998). Therefore, the use of workshops or courses on critical appraisal, in collaboration with other dissemination strategies may be effective among public health decision-makers.

The final important finding in this study that must be used in the development of a comprehensive dissemination strategy was the significant association between the perception that local politicians were influenced by research evidence and the inflience of the systematic overviews on program justification decisions. This finding suggested that the dissemination strategy should also consist of activities that assist public health decision-makers in presenting the results of systematic overviews to local politicians, agencies, community groups and possibly 
even community residents. This suggestion is supported in the literature which has stressed the importance of including policy makers from varying levels in the dissemination and utilization process (Ingram, 1996; Lomas, 1993c). Therefore, the final recommendation for the dissemination strategy would be to package the systematic overviews in a variety of ways that are appropriate for use not only among public health decision-makers, but for a variety of audiences that are involved in making decisions concerning public health services.

The comprehensive dissemination strategy proposed in this dissertation is specific to the needs and practices of decision-makers in public health in Ontario. It encompasses a wide variety of activities that are directed at perceptions of the characteristics of the innovation, organization, individuals and the environment. It also includes activities that are directed not only at public health decision-makers but also at those who make decisions concerning the provision of public health services, particularly local politicians. By addressing research dissemination in such an holistic fashion, the utilization of systematic overviews might be improved in the future across the province.

\subsection{Recommendations for Future Research}

The implementation of the dissemination strategy proposed in this study would set up an opportunity to test a number of hypotheses related to the effectiveness of dissemination strategies, that are based on public health decision-maker's perceptions of the innovation, the organization and individuals. However, many of these hypotheses would represent complex research questions that may not be easily answered, particularly given the small sample size available for study in public health. Therefore, future evaluation of these dissemination strategies may be best examined using a more qualitative approach, focusing on answering why and how certain dissemination strategies work and others do not. In addition future research should focus 
on understanding how public health decision-makers form perceptions of innovations, and how changes in perceptions can be facilitated. Further exploration of the decision-making process, at both the individual and organizational level, as well as where systematic overviews and research evidence fit into this process will help identify more specific dissemination strategies. Further research is needed to identify and operationalize measures of research utilization that are more objective and less reliant on respondent recall. Finally, additional research is needed to define characteristics of public health units that can be measured at the organizational level as opposed to attempting to aggregate individual level data. These types of measures will facilitate comparisons across public health units in Ontario in addition to decision-makers.

\subsection{Ethical Issues and Considerations}

The potential to cause harm to respondents, third party respondents or the target population was relatively small in this study. However, to minimize/reduce risk of harm two steps were taken. In order to minimize any perceived pressure that respondents might have experienced to participate in the study the introductory letter stressed that participation was voluntary. The second step related to confidentiality. It would have been unethical to report the results in a way that would lead to specific public health units or decision-makers within health units being identified, when their participation in the study was confidential. This problem was eliminated by informing all respondents that the lowest level of data analysis would be at the group level, for example, as urban or rural health units or according to specific staff groups such as medical officers of health or program managers.

\subsection{Limitations of the Study}

There were several limitations of the sampling design, such as generalizability, missing respondents in the sampling frame, and small sample sizes in some cells. One important 
limitation of this design was the lost to follow-up rate. During the two years between the dissemination of the systematic overviews and this current study, many changes to the public health sector occurred, resulting in more than 40 decision-makers not being available for followup. Therefore, even though the response rate was high in this study, there were some issues with the number of decision-makers who were lost to follow-up. However, no significant differences on a number of individual characteristics were found between those who participated in this study and those who had left public health between 1996 and 1998.

Although most health units wanted to be involved in this study, one issue that arose was that health units wanted to have just one representative answer the questionnaire on behalf of the health unit as opposed to all appropriate decision-makers. They also felt it was important to have someone answer the questionnaire who had the most experience so that the best answers were provided. Again it was stressed in the introductory letter that the participation of all those involved in making decisions about public health practice, regardless of experience was needed. Also, health units were encouraged to have as many decision-makers as possible participate in the study.

Given the sampling frame and target population another limitation of the sampling design was related to how generalizable the results would be to the public health field in general in Ontario. The results of this study were only directly generalizable to public health professionals who make decisions for public health practice. However, since this included public health professionals from all the public health divisions, it is likely that the findings were generalizable to public health decision-makers throughout Ontario. This may not necessarily be the case for public health decision-makers outside of Ontario. 
There were relatively small sample sizes in this study. However, the alternative of increasing the sample size would have meant expanding the sample to include public health units from outside Ontario. It was believed that including public health units from other provinces, with different public health roles and responsibilities would result in significantly more methodological concerns than currently existed. In addition to a small sample there was also an issue related to data not being collected on all participants, resulting in different numbers of cases being included in the six multiple regression analyses. Since respondents in 1996 who had not used a systematic overview during the three-month follow-up period were not asked questions pertaining to the usefulness of the systematic overviews, 53 of the 141 cases had incomplete data. One method to overcome different cases being analyzed for the six outcome variables would be to remove all cases from the analysis that did not have complete data. Therefore, the maximum number of cases to be analyzed in this study would have been 88 .

There were also some concerns that decision-makers within health units would have more similar responses than those between health units, resulting in a clustering effect. In many instances adjustments for this clustering effect should be used in regression analyses. In this study however, within health unit variability was often times greater than the between health unit variability, suggesting the a clustering effect was not present, and therefore no adjustments were necessary for the regression analysis. One reason why the within variability may have been greater than the between variability, was related to how the questions were asked. In all instances respondents were asked about their personal perceptions of organizational and environmental characteristics, as opposed broader questions focused on the perception of the organization as a whole. Therefore, a clustering effect was not a major concern in this study. The use of a large number of independent variables combined with a fairly small sample size was also a limitation 
of this study. This large number of variables may have resulted in some variables being found significant due to chance alone. The use of scaled items, as opposed to individual variables would have decreased the overall number of analyses conducted in this study, thereby reducing Type Il errors. However, since this was the first time data on these characteristics were collected, it was believed that it was more important to examine the individual relationships between the independent and dependent variables. Future studies should however, examine the development of scales for the various types of characteristics, such as characteristics of the innovation. organization and the environment.

There were also some limitations with using a telephone administered questionnaire. Although the length of the questionnaire was kept to a minimum, the objectives of this research study were such that it was necessary to ask numerous questions. The approximate time of 20 minutes might have been too great a time commitment and may have resulted in some respondents refusing to complete the questionnaire. As well, respondents might not have provided as complete responses as possible, or might have been more inclined to answer 'don't know', or might have become less interested as the survey progressed and given less thought to questions at the end of the survey. To ensure these issues did not arise the researcher clearly identified the length of the survey and directed the respondent to choose an interview time that allowed them to think through some complex issues.

Recall bias was another important limitation of this study. The time period of two years, between the dissemination of the systematic overviews and the data collection phase of this study might have resulted in some respondents being unable to reliably and accurately recall their actions during this time. It was difficult to determine from this questionnaire, what the "true" value for these questions would be without this bias. 
Another possible limitation of this study involved the collection of data on the characteristics of the innovation two years before data on the outcomes, and organizational and environmental characteristics were collected. Although only data from those individuals who participated in the 1998 study were merged with those who participated in the OHCEN study in 1996. there are some problems associated with this approach. Most notably is that decisionmaker's perceptions of the characteristics of the innovation may have become more positive from the time the data was collected in the OHCEN study in 1996, and the time at which they used the systematic overviews. It is unknown if decision-maker's perceptions become more positive sometime during the two year follow-up. It may be that this more positive perception in fact led to the overviews being used, and not the level reported in this study. The result could be that the association between the innovation characteristics and the research utilization outcomes might be stronger than that observed in the study. However, perceptions of the innovation were generally very positive at the time the data were collected in 1996 , with very little room left for more positive perceptions to be measured. Therefore, if respondent's perceptions of the innovation did become more positive, the effect on the strength of the association between these variables and the outcome variables would have been minimal.

The final two limitations are related to social desirability bias. The respondents might have responded in ways they thought the interviewer wanted them to. As well, previous work with this target population suggested that many respondents were hesitant to say negative things about their public health unit, and therefore the responses might have overestimated their evidence-based decision-making. The researcher attempted to overcome this issue by stressing the results of the study would be kept confidential and would not be reported according to specific public health units or individuals. 


\subsection{Conclusions}

This study focused specifically on identifying characteristics of the innovation, organization, environment and the individual, that were believed to be associated with research utilization among public health decision-makers in Ontario. It also considered these variables in explaining variation in the use of disseminated research evidence. This was the first study in public health to measure characteristics of the innovation, organization and the environment. and to examine the relationships between these variables and various measures of research utilization. It was also the first time in the public health setting that alternative measures of research utilization, (other than the traditional dichotomous measure of "use versus no use") have been examined. The most important findings to emerge from this study was the importance of innovation characteristics such as, the perceived relevance of the systematic overviews to decisions being made, and whether they are perceived as being easy to use. In addition the perception that one's public health unit values the use of research evidence in decision-making was also an important predictor of the extent to which systematic overviews influenced public health decisions. The findings and interpretation of this study provided direction and guidance in the recommendation for future dissemination strategies in public health. As well this study identified areas of future research not only in public health but also in the health care field, with respect to research dissemination and utilization. 


\subsection{References}

Abrahamson, E. (1991). Managerial fads and fashions: The diffusion and rejection of innovations. Academy of Management Review, 16(3), 586-612.

Abrahamson, E., \& Rosenkoff, E. (1993). Institutional and competitive bandwagons: Using mathematical modeling as a tool to explore innovation diffusion. Academy of Management Review, 18(3), 487-517.

Allery, L.A., Owen, P.A., \& Robling, M.E. (1997). Why general practitioners and consultants change their clinical practice: A critical incident study. British Medical Journal. $314,870-874$.

Anderson. J.G., \& Jay, S.L. (1985). The diffusion of medical technology: Social network analysis and policy research. The Sociological Quarterly, 26, 49-64.

Angell, M., \& Kassierer, J.P. (1994). Clinical research: What should the public believe? New England Joumal of Medicine, 331, 189-190.

Armitage, S. (1990). Research utilization in practice. Nurse Education Today, 10(1), 10-15.

Ashcroft. T., \& Kristjanson, J. (1994). Research utilization in Maternal-Child Nursing: Application of the CURN model. Canadian Journal of Nursing Administration, 7(3), 90102.

Baessler. C.A., Blumberg, M., \& Cunningham, J.S. (1994). Medical-surgical nurses' utilization of research methods and products. Medsurgical Nursing, 3, 113-121.

Baldridge, J.V., \& Bumham, R.A. (1975). Organizational innovation: Individual, organizational, and environmental impacts. Administrative Science Quarterly, 20, 165176.

Basch, C.E., Eveland, L.D., \& Portnoy, B. (1986). Diffusion systems for education and learning about health. Family and Community Health, 9(2), 1-26.

Battista. R.N. (1989). Innovation and diffusion of health-related technologies. International Journal of Technology Assessment in Health Care, 5, 227-248.

Becker, M.H. (1970a). Factors affecting diffusion of innovations among health professionals. American Journal of Public Health, 60(2), 294-304.

Becker, M.H. (1970b). Sociometric location and innovativeness: Reformation and extension of the diffusion model. American Sociological Review, 35, 267-281.

Bero, L.A., \& Jadad, A.R. (1997). How consumers and policymakers can use systematic reviews for decision-making. Annals of Internal Medicine, 127(1), 37-42. 
Bigoness, W.J., \& Perreault, Jr.W.D. (1981). A conceptual paradigm and approach for the study of innovators. Academy of Management Journal. 24(1), 68-82.

Birch. S. As a matter of fact: Evidence-based decision-making 'unplugged'. (1997). McMaster University Centre for Health Economics and Policy Analysis Working Paper. Hamilton, 97-2.

Bohannon, R.W., \& LeVeau, B.F. (1986). Clinicians' use of research findings: A review of literature with implications for physical therapists. Physical Therapy, 66(1), 45-50.

Bostrum, J., \& Sutter, W. (1993). Research utilization: Making the link to practice. Journal of Nursing Staff Development, 9(1), 28-34.

Brown, G.D. (1995). Understanding barriers to basing nursing practice upon research: $\mathbf{A}$ communication model. Ioumal of Advanced Nursing, 21. 154-157.

Brown. L.A. (1981). Innovation diffusion: A new perspective. New York: Methuen.

Burns, L.R., \& Wholey, D.R. (1997). Adoption and abandonment of matrix management programs: Effects of organizational characteristics and interorganizatonal networks. Academy of Management Journal, 36(1), 106-138.

Burt, R. (1987). Social contagion and innovation: Cohesion versus structural equivalence. American Journal of Sociology, 92, 1287-1335.

Butler, L. (1995). Valuing research in clinical practice: A basis for developing a strategic plan for nursing research. Canadian Journal of Nursing Research, 27(4), 33-49.

Calsyn. R.J., \& Tornatzky, L.G. (1977). Incomplete adoption of an innovation: The case of goal attainment scaling. Evaluation, 4, 127-130.

Cameron. K.S., \& Freeman, S.J. (1991). Research in organizational change and development. Greenwich, Conn: JAI Press Inc.

Cameron, R., Brown, S., \& Best, J.A. (1996). The dissemination of chronic disease prevention programs: Linking science and practice. Canadian Journal of Public Health, S7. S50-S53.

Carter, C.F., \& Williams, B.R. (1957). Industry and technological progress. London: Oxford University Press.

Cavanagh, S.J., \& Coffin, D. (1993). Teaching nursing research. Senior Nurse, 13(4), 51 54.

Cavanagh, S.J.. \& Tross, G. (1996). Utilizing research findings in nursing: Policy and practice considerations. Journal of Advanced Nursing, 24(5), 1083-1088. 
Champion. V.L., \& Leach, A. (1989). Variables related to research utilization in nursing: An empirical investigation. Journal of Advanced Nursing, 14, 705-710.

Ciliska, D., Hayward, S., Thomas, H., Mitchell, A., Dobbins, M., Underwood, E.J., Rafael. A., \& Martin, E. (1996). A systematic overview of the effectiveness of home visiting as a delivery strategy for public health nursing interventions. Canadian Journal of Public Health, 87(3), 193-I 98 .

Ciliska. D., Hayward. S., Underwood, I., \& Dobbins, M. (1999). Transferring public health nursing research to health system planning: Assessing the relevance and accessibility of systematic overviews. Canadian Journal of Nursing Research, 31 (1), 23-36.

Closs, S.J., \& Cheater, F.M. (1994). Utilization of nursing research: Culture, interest and support. Joumal of Advanced Nursing, 19, 762-773.

Cockerill, R., \& Barnsley, J. (1996) Innovation theory and its applicability to our understanding of the diffusion of new management practices in health care organizations. (un pub)

Coeling, H., \& Simms, L. (1993). Facilitating innovation at the nursing unit level through cultural assessment: Part 2. Journal of Nursing Administration, 23(5), 13-20.

Coleman, J.S., Katz, E., \& Menzel, H. (1966). Medical innovation: A diffusion study. Indianapolis: Bobbs-Merrill.

Conn. V.S., \& Armer, J.M. (1996). Meta-analysis and public policy: Opportunity for nursing impact. Nursing Outlook, 44 (6), 267-271.

Coyle, L.A., \& Gallino Sokop, A. (1990). Innovation adoption behaviour among nurses. Nursing Research, 39(3). 176-180.

Crane, J. (1995). The future of research utilization. Nursing Clinics of North America, 30(3). 565-577.

Cronenwett, L.R. (1995). Effective methods for disseminating research findings to nurses in practice. Nursing Clinics of North America, 30(3), 429-438.

Dalrymple, P.W. (1990). CD-ROM Medline use and users: Information transfer in the clinical setting. Bulletin of the Medical Library Association, 78(3), 224-232.

Damanpour, F. (1991). Organizational innovation: A meta-analysis of effects of determinants and moderators. Academy of Management Review, 34(3), 540-555.

Davis, D.A., \& Taylor-Vaisey, A. (1997). Translating guidelines into practice: A systematic review of theoretic concepts, practical experience and research evidence in the adoption of clinical practice guidelines. Canadian Medical Association Journal, 157(4), 408-416. 
Davis, D.A., Thomson, M.A., Oxman, A.D., \& Haynes, R.B. (1992). Evidence for the effectiveness of CME: A review of 50 randomized controlled trials. Journal of the American Medical Association, 268(9), $1111-1117$.

Davis, D.A., Thomson, M.A., Oxman, A.D., \& Haynes, R.B. (1995). Changing physician performance: A systematic review of the effect of continuing medical education strategies. Journal of the American Medical Association, 274(9), 700-705.

Deal, T.. \& Kennedy, A. (1982). Corporate cultures: The rites and rituals of corporate life. Reading, MA: Addison-Wesley.

Dean. J.W., \& Sharfman, M.P. (1993). Procedural rationality in the strategic decision-making process. Journal of Management Studies, 30(4), 587-610.

Denison. D.R., \& Spreitzer, G.M. (1991). Research in organizational change and development. Greenwich: JAI Press Inc.

Dobbins, M., Ciliska, D., \& DiCenso, A. Dissemination and use of research evidence for policy and practice: A framework for developing, implementing and evaluating strategies. (1998). Ottawa: The Dissemination and Utilization Model Advisory Committee.

Dobbins, M., Thomas, H., Ciliska, D., Hayward, S., Ploeg, J., \& Underwood, E.J. The effectiveness of community-based heart health projects: A systematic overview. (1996). McMaster University - University of Toronto Hamilton, ON: Quality of Nursing Worklife Research Unit Working Paper Series. 96-1.

Downs, G.W.Jr., \& Mohr, L.B. (1976). Conceptual issues in the study of innovation. Administrative Science Quarterly, 21 , 700-714.

Estabrooks, C.A. (1998). Will evidence-based nursing practice make practice perfect. Canadian Journal of Nursing Research, 30(1), 15-36.

Eysenck, H.J. (1994). Meta-analysis and its problems. British Medical Journal, 309(6957), 789-792

Feinstein, A.R. (1996). Multivariable analysis: an introduction. New Haven: Yale University Press.

Ferrence, R. (1996). Using Diffusion theory in Health Promotion: The case of tobacco. Canadian Journal of Public Health, 87(2), S24-27.

Freiman, M.P. (1985). The rate of adoption of new procedures among physicians. Medical Care, 23(8), 939-945.

French, W.L., , \& . (1984). Organization development. (3 ed.). Englewood Cliffs, NJ: Prentice Hall. 
Funk, S.G., Champagne, M.T., Wiese, R.A., \& Tornquist, E.M. (1991 a). Barriers: The barriers to research utilization scale. Applied Nursing Research, 4, 39-45

Funk, S.G., Champagne, M.T., Wiese, R.A., \& Tornquist, E.M. (199lb). Barriers to using research findings in practice: The clinician's perspective. Applied Nursing Research, $4(2) .90-95$.

Funk, S.G., Tornquist, E.M., \& Champagne, M.T. (1995). Barriers and facilitators of research utilization: An integrative review. Nursing Clinics of North America, 30(3), $395-406$.

Ganong. L. (1987). Integrative reviews on nursing research. Research in Nursing \& Health. $10,1-11$.

Gelijins, A., \& Rosenburg, N. (1994). The dynamics of technological change in medicine. Health Affairs, Summer, 28-46.

Gennaro, S. (1994). Research utilization: An overview. Journal of Obstetrics and Gynecological Nursing, 23(4), 313-319.

Goode, C.J., Butcher, L.A., Cipperley, L., Ekstom, J., Gosch, B., Hayes, J., Lovett, M., \& Wellendorf, S. (1991). Research utilization: A study guide. Ida Grove: Home Video Productions.

Granovetter, M. (1985). Economic action and social structure: The problem of embeddedness. American Journal of Sociology, 91, 481-510.

Gray, J.A.M. (1997). Evidence-based healthcare: How to make health policy and management decisions. New York: Churchill Livingstone.

Greenwood. J. (1984). Nursing research: A position paper. Journal of Advanced Nursing, 9(1). 77-82.

Greer. A. (1977). Advances in the study of diffusion of innovation in health care organizations. Millbank Memorial Fund Quarterly/Health and Society, Fall, 505-533.

Grilli, R., \& Lomas, J. (1994). Evaluating the message: The relationship between compliance rate and the subject of a practice guideline. Medical Care, 32, 202-213.

Gustafson, D.H., \& Schoofs Hundt, A. (1995). Findings of innovation research applied to quality management principles for health care. Health Care Management Review, 20(2), 16-33.

Hage, J. (1974). A systems perspective on organizational program change. In A. D. Kaluzny, J. T. Gentry. \& J. E. Veney (Eds.), Innovation in health care organizations. Chapel Hill: Department of Health Administration, Liniversity of North Carolina. 
Hage, J., \& Aiken, M. (1967). Program change and organizational properties. American Journal of Sociology. 72, 503-510.

Hage, J., \& Dewar, R (1973). The prediction of organizational performance: The case of program innovation. Administrative Science Quarterly, 18, 279-285.

Haines, A., \& Jones, R. (1994). Implementing findings of research. British Medical Journal, 308(4 June). 1488-1492.

Halpert, H.P. (1966). Communications as a basic tool in promoting utilization of research findings. Community Mental Health Joumal, 2(3), 231 -236.

Hayward, S., Ciliska, D., DiCenso, A., Thomas, H., Underwood, E.J., \& Rafael, A. (1996a). Evaluation research in public health: Barriers to the production and dissemination of outcomes data. Canadian Journal of Public Health, 87(6), 413-417.

Hayward, S., Ciliska, D., Mitchell, A., Thomas, T., Underwood, E.J., \& Dobbins, M. Effectiveness of public health nursing interventions in parent-child health: $A$ systematic overview of literature reviews. (1996b). Hamilton, McMaster University-University of Toronto: Quality of Nursing Worklife Research Unit. 96-2.

Heater, B.S., Becker, A.M., \& Olson, R.K. (1988). Nursing interventions and patient outcomes: A meta-analysis of studies. Nursing Research, 37(5), 303-307.

Hicks. C. (1996). A study of nurses' attitudes towards research: A factor analytic approach. Journal of Advanced Nursing, 23(2), 373-379.

Hofstede, G. (1980). Culture's consequences: International differences in work-related values. Beverly Hills: Sage.

Horsley, J.A. Crane, J., \& Crabtree, M.K. (1983). Using research to improve nursing practice: A guide. New York: Grune \& Stratton.

Hosmer, D.W.Jr., \& Lemeshow, S. (1989). Applied Logistic Regression. New York: John Wiley \& Sons.

Hunt, J. (1981). Indicators for nursing practice: The use of research findings. Joumal of Advanced Nursing, 6, 189-1 94.

Hunt, J.M. (1996). Barriers to research utilization. Journal of Advanced Nursing, 23, 423425 .

Ingram. N. (1996). The research basis of health care decision-making. Journal of Advanced Nursing, 23, 692-696.

Jennett, P.A., \& Premkumar, K. (1996). Technology-based dissemination. Canadian Journal of Public Health, 87(Supplement 2), S34-S39. 
Johnson. J.L., Green, L.W., Frankish, C.J., Maclean, D.R., \& Stachenko, S. (1996). A dissemination research agenda to strengthen health promotion and disease prevention. Canadian Journal of Public Health, 87, S5-S10

Kaluzny, A.D. (1974). Innovation in health services: Theoretical framework and review of research. Health Services Research, (Summer), 101-120.

Kaluzny, A.D. (1987). New perspectives in organizational theory and its application to health services organizations. Medical Care, 44(2), 227-233.

Kaluzny, A.D., \& Veney, J.E. (1973). Attributes of health services as factors in program implementation. Journal of Health and Social Behaviour, 14(June), 124-133.

Kaluzny, A.D., Veney, J.E., \& Gentry, J.T. (1974). Innovation of health services: A compartive study of hospitals and health departments. Millbank Memorial Fund Quarterly /Health and Society, Winter, 51-81.

Kenny, N.P. (1997). Does good science make good medicine: Incorporating evidence into practice is complicated by the fact that clinical practice is as much art as science. Canadian Medical Association Journal, 157(1), 33-36.

Kimberly, J.R. (1978). Hospital adoption of innovation: The role of integration into external informational environments. Journal of Health and Social Behaviour, 19(December), $361-373$.

Kimberly, J.R., \& Evanisko, M.J. (1981). Organizational innovation: The influence of individual, organizational, and contextual factors on hospital adoption of technological and administrative innovations. Academy of Management Journal, 24(4), 689-713.

Kitson, A., Ahmed, L.B., Harvey, G., Seers, K., \& Thompson, D.R. (1996). From research to practice: One organizational model for promoting research-based principles. Journal of Advanced Nursing, 23, 430-440.

Kotter, J.P. (1979). Managing external dependence. Academy of Management Review, $4(1), 87-92$.

Kouri, D. (1996) Introductory module: Introduction to decision theory and practice. Saskatoon. Heaith Services Evaluation Research Unit.

Lacey, E.A. (1994). Research utilization in nursing practice: A pilot study. Journal of Advanced Nursing, 19(5), 987-995.

Langley, A., Mintzberg, H., Pitcher, P., Posada, E., \& Saint-Macary, J. (1995). Opening up decision-making: The view from the black stool. Organization Science, 6(3), 260-279.

Larsen, J.K., \& Agarwalla-Rogers, R. (1977). Re-invention of innovative ideas: Modified? Adopted? None of the above. Evaluation, 4, 136-140. 
Last, J.M. (1995). A dictionary of Epidemiology. (3 ed.). New York: Oxford Liniversity Press.

Lewis, L.K., \& Seibold, D.R. (1993). Innovation modification during intraorganizational adoption. Academy of Management Review, 18(2), 322-354.

Lomas, J. (1990). Finding audiences, changing beliefs: The structure of research use in Canadian health policy. Joumal of Health Politics, Policy and Law, 15 (3), 525-542.

Lomas, J. (1991). Words without action? The production, dissemination, and impact of consensus recommendations. Annual Review of Public Health, 12, 41-65.

Lomas, J. (1993a). Diffusion, dissemination, and implementation: Who should do what? Annals of the New York Academy of Science, 703, 326-235.

Lomas, J. (1993b). Retailing research: Increasing the role of evidence in clinical services for childbirth. The Milbank Quarterly, 71(3), 439-475.

Lomas, J. (1993c). The role of opinion leaders in educational strategies. Australian Prescriber, 16(Suppl 1), 25-30.

Lomas. J. Improving research dissemination and uptake in the health sector: Beyond the sound of one hand clapping. (1997). Hamilton: Advisory Committee on Health Services to the Federal/Provincial/ Territorial Conference of Deputy Ministers.

Lomas. J., \& Haynes, R.B. (1988). A taxonomy and critical review of tested strategies for the application of clinical practice recommendations: From official to individual clinical policy. American Journal of Preventive Medicine, 1(Supplement 2), 77-94.

Luker, K.A., \& Kendrick, M. (1992). An exploratory study of the sources of influence on the clinical decisions of community nurses. Journal of Advanced Nursing, 17, 457-466.

MacGuire, J.M. (1990). Putting nursing research findings into practice: research utilization as an aspect of the management of change. Journal of Advanced Nursing, 15, 614-620

Maclean, D.R. (1996). Positioning dissemination in public health policy. Canadian Journal of Public Health. 87, S40-S43.

MacPhail, J. (1997). Nursing research as a basis for practice in community health nursing. In An introduction to issues in community health nursing in Canada. (pp. 81-96).

Mark. B.A. (1996). Organizational Culture. Annual Review of Nursing Research, 14, 145163.

Marshall, J.G. (1993). Issues in clinical information delivery. Library Trends, 42(1), 83107. 
McKinney, M.M., Kaluzny, A.D., \& Zuckerman. H.S. (1991). Paths and pacemakers: Innovation diffusion networks in multi-hospital systems and alliances. Health Care Management Review, 16(1), 17-23.

McSherry, R. (1997). What do registered nurses and midwives feel and know about research. Journal of Advanced Nursing, 25(5), 985-998.

McSkimming, S.A. (1996). Issues in clinical nursing research: Creating a cultural norm for research and research utilization in a clinical agency. Western Journal of Nursing Research, 18(5), 606-610.

McWilliam, C.L., Desai, K., \& Greig, B. (1997). Bridging town and gown: Building research partnerships between community-based professional providers and academia. Journal of Professional Nursing, $13(5), 307-315$.

Meah. S., Luker, K.A., \& Cullum, N.A. (1996). An exploration of midwives' attitudes to research and perceived barriers to research utilization. Midwifery, 12, 73-84.

Meyer, A.D. \& Goes. J.B. (1988). Organizational assimilation of innovation: A multilevel contextual analysis. Academy of Management Journal, 31(4), 897-923.

Michel, Y.. \& Sneed, N.V. (1995). Dissemination and use of research findings in nursing practice. Journal of Professional Nursing, $11(5), 306-311$.

Milio, N. (1971). Health care organizations and innovation. Journal of Health and Social Behaviour, 12(June), 163-173.

Miller, J.R., \& Messenger, S.R. (1978). Obstacles to applying nursing research findings. American Journal of Nursing, 78, 632-634.

Mintzberg, H., Raisinghani, D., \& Theoret, A. (1976). The structure of "unstructured" decision processes. Administrative Science Quarterly, 21, 246-275.

Mirvis, P.H., \& Berg, D.N. (1977). Failures in organizational development and change. New York: Wiley Interscience.

Mott, P. (1972). The characteristics of effective organizations. New York: Harper \& Row.

Mulhall, A. (1992). Nursing research, exploring the options. Nursing Standard, 7(3), 35-36.

Nelson, R.R., \& Yates, D. (1978). Innovation and implementation in public organizations. Lexington: Lexington Books.

Norman, G.R., \& Shannon, S.I. (1998). Effectiveness of instruction in critical appraisal (evidence-based medicine) skills: A critical appraisal. Canadian Medical Association Journal, 158(2), 177-181.

Norusis, M.J. (1997). SPSS Professional Statistics 7.5. Chicago: SPSS Inc. 
Nutbeam, D. (1996). Improving the fit between research and practice in health promotion: Overcoming structural barriers. Canadian Journal of Public Health, 87, S18-S23.

Nutt, P.C. (1984). Types of organizational decision processes. Administrative Science Quarterly, 29, 4I 4-450.

Ontario Ministry of Health. Mandatory Health Programs and Services Guidelines. (1989). Toronto: Queen's Printer for Ontario.

Orlandi, M.A. (1996). Health promotion technology transfer: Organizational perspectives. Canadian Journal of Public Health, 87(2), S28-S33.

Oxman, A.D., Thomson, M.A., Davis, D.A., \& Hayes, J.E. (1995). No magic bullets: A systematic review of 102 trials of interventions to improve professional practice. Canadian Medicai Association Journal, 153(10), 1423-1431.

Patel, V.L. (1996). Cognition and technology in health education research. Canadian Journal of Public Health, 87(Supplement 2), S63-S67.

Pearson. C.A. (1992). Autonomous workgroups: An evaluation at an industrial site. Human Relations, 45(9), 905-936.

Pettengill, M.M., Dillies, D.A., \& Chambers Clark, C. (1994). Factors encouraging and discouraging the use of nursing research findings. IMAGE: Journal of Nursing Scholarship, 26(2), 143-147.

Pettigrew, A. (1979). On studying organizational cultures. Administrative Science Quarterly, 24. 570-580.

Ploeg, J., Ciliska, D., Dobbins, M., Hayward, S., Thomas, H., \& Underwood, E.J. A systematic overview of the effectiveness public health nursing interventions: An overview of adolescent suicide prevention programs. (1995a). McMaster University University of Toronto Hamilton, ON. Quality cí Nursing Worklife Research Unit. 9512.

Ploeg. J., Dobbins, M., Hayward, S., Ciliska, D., Thomas, H., \& Underwood, E.J. A systematic overview of community development projects. (1995b). McMaster University - University of Toronto, Hamilton, ON. Quality of Nursing Worklife Research Unit working Paper Series. 95-5.

Poole, M.S., \& DeSanctis, G. (1990). Understanding the use of group decision support systems: The theory of adoptive structuration. In J. Fulk \& C. Stienfield (Eds.), Organizations and communication technology. (pp. 173-193). Newbury: Sage.

Power, E.J., Tunis, S.R., \& Wagner, J.L. (1994). Technology assessment and public health. Annual Review of Public Health, 15, 561-579. 
Pranulis, M.F., \& Driever, M.J. (1990). A conceptual framework for analyzing influences on research productivity in clinical settings. Western Journal of Nursing Research, 12,563565.

Preisser, J.S., \& Koch, G.G. (1997). categorical data analysis in public health. Annual Review of Public Health, 18, 51-82.

Reichers, A., \& Schneider, B. (1990). Climate and culture: An evolution of concepts. In B. Schneider (Ed.), Organizational climate and culture. (pp. 5-39). San Francisco: JosseyBass.

Rodgers, S. (1994). An exploratory study of research utilization by nurses in general medical and surgical wards. Journal of Advanced Nursing, 20, 904-911.

Rogers, E.M. (1983). The innovation-decision process. In Anonymous, Diffusion of Innovations. (pp. 163-209). London: Collier Macmillan Publishers.

Rogers, E.M. (1988). Information technologies: How organizations are changing. In G. M. Goldhaber \& G. A. Barnett (Eds.), Handbook of organizational communication. (pp. 437-452). Norwood: Ablex.

Rogers. E.M. (1995). Diffusion of Innovations. (4th ed.). New York: The Free Press.

Rogers, E.M., \& Shoemaker, F.F. (1971). Communication of innovations: A cross-cultural approach. New York: Free Press.

Royle, J.A., Blythe, J., DiCenso, A., Baumann, A. \& Fitzgerald, D. (1997). Do nurses have the information resources and skills for research utilization. Canadian Joumal of Nursing Administration, (Sept/Oct), 9-30.

Royle, J.A.. Blythe, J., Ingram, C., DiCenso, A., Bhatnager, N., \& Potvin, C. (1996). The research utilization process: The use of guided imagery to reduce anxiety. Canadian Oncology Nursing Journal, 6(1), 20-25.

Sackett, D., Rosenberg, W.M., Gray, J.A.M., Haynes, R.B., \& Richardson, W.S. (1996). Evidence-based medicine: What it is and what it isn't. British Medical Joumal, 312, 71 72.

Scott, W.R. (1990). Innovation in medical care organizations: A synthetic review. Medical Care Review, 47(2), 165-192.

Scott. W.R. (1993). The organization of medical care services: Toward and integrated theoretical model. Medical Care Review, 50(3), 271-303.

Shortell, S.M., Morrison, E.M., \& Friedman, B. (1989). Strategic choices for America's hospitals: Managing change in turbulent times. San Francisco: Josse-Bass. 
Shortell. S.M., Rpisseai, D.M., Gillies, R.R., Devers, K.J., \& Simons, T.L. (1991). Organizational assessment in intensive care units (ICUs): Construct development, reliability, and validity on the ICU nurse-physician questionnaire. Medical Care, 29(8), 709-724.

Sibbald, B., \& Roland, M. (1997). Getting research into practice. Journal of Evaluation in Clinical Practice, 2(4), 15-21.

Soumerai, S.B., \& Avom, J. (1990). Principles of educational outreach (Academic Detailing) to improve clinical decision making. Joumal of the American Medical Association. 263(4). 549-556.

Soumerai, S.B., McLaughlin. T.J., \& Avorn. J. (1989). Improving drug prescribing in primary care: A critical analysis of the experimental literature. The Milbank Quarterly, 67(2), 268-317.

SPSS Inc. (1998). SPSS Base 8.0: Applications Guide. Chicago:

Stetler, C.B. (1994). Refinement of the Stetler/Marram model for application of research findings to practice. Nursing Outlook, 42(1), 15-25.

Stolk. B.J., \& Mayo, E. (1995). Barriers to research utilization perceived by staff public health nurses. University of Western Ontario;

Strang, V.R, \& Pearson, J. (1995). Factors influencing the utilization of results: A case study of an evaluation of an adult day care program. The Canadian Joumal of Program Evaluation, $10(1)$. 73-87.

Swanson, J.M., Albright, J., Steirn, C., Schaffner, A., \& Leslie, C. (1992). Program efforts for creating a research environment in a clinical setting. Western Journal of Nursing Research, 14. 241-245

Thomas, C., Ward, M., Chorba, C., \& Kumiega, A. (1990). Measuring and interpreting organizational culture. Journal of Nursing Administration, 20(6), 17-24.

Thomson, M.A., Oxman, A.D., Davis, D.A., Freemantle, N., \& Harvey, E.L. (1997) Outreach visits to improve health professional practice and health care outcomes. The Cochrane Library, (4), 1-15.

Titler, M.G., Klieber, C., \& Steelman, V. (1994). Infusing research into practice to promote quality care. Nursing Research, 43, 307-313

Tornatzky, L.G., \& Klein, K.J. (1981). Innovation characteristics and innovation adoptionimplementation: A meta-analysis of findings. Washington, D.C.: National Science Foundation, division of Industrial Science and Technological Innovation.

Umlauf, M.G., \& Sherman, S. (1992). Facilitating research utilization through collaboration. Nursing Connections, 5(2), 37-42. 
Utterback. J.M. (1974). Innovation in industry and the diffusion of technology. Science, 183(February), 620-626.

Van Ess Coeling, H., \& Simms, L.M. (1993a). Facilitating innovation at the nursing unit level through cultural assessment, Part $\mathrm{I}$ : How to keep management ideas from falling on deaf ears. Journal of Nursing Administration, 23(4), 46-53.

Van Ess Coeling, H., \& Simms, L.M. (1993b). Facilitating innovation at the unit level through cultural assessment, Part 2: Adapting managerial ideas to the unit work group. Journal of Nursing Administration, 23(5), 13-20.

Varcoe, C., \& Hilton, A. (1995). Factors affecting acute-care nurses' use of research findings. Canadian Journal of Nursing Research, 27(4), 51-71.

Veeramah, V. (1995). A study to identify the attitudes and needs of qualified staff concerning the use of research findings in clinical practice within mental health care settings. Journal of Advanced Nursing, 22, 855-861.

Veney, J.E., Kaluzny, A.D., Gentry, J.T., Sprague, J.B., \& Duncan, D.P. (1971). Implementation of health programs in hospitals. Health Services Research, 6, 350-355.

Waddell, D.L. (1991). The effects of continuing education on nursing practice: A metaanalysis. The Journal of Continuing Education in Nursing, 22(3), 113-118.

Walczak. J.R., McGuire, D.B., Haisfield, M.E., \& Beezley, A. (1994). A survey of research related activities and perceived barriers to research utilization among professional oncology nurses. Oncology Nursing Forum, 21(4), 710-719.

Warner, K.E. (1975). A "desperation-reaction" model of medical diffusion. Health Services Research, Winter, 369-383.

White. J.M., Leske, J.S., \& Pearcy, J.M. (1995). Models and processes of research utilization. Nursing Clinics of North America, 30(3), 409-438.

Worrall, G., Chaulk, P., \& Freake, D. (1997). The effects of clinical practice guidelines on patient outcomes in primary care: A systematic review. Canadian Medical Association Journal, 156(12), 1705-1712.

Zaltman, G., Duncan, R., \& Holbeck, J. (1973). Innovations and organizations. New York: John Wiley. 


\subsection{Tables}


Table 1: Variable Definition and Measurement

\begin{tabular}{|c|c|c|}
\hline Variable Label & Operational Definition & Data Source \\
\hline \multicolumn{3}{|l|}{$\begin{array}{l}\text { Organizational } \\
\text { Characteristics }\end{array}$} \\
\hline $\begin{array}{l}\text { Size } \\
(Q 11315)\end{array}$ & $\begin{array}{l}\text { Number full time equivalent employees } \\
\text { ( } 35 \mathrm{hrs} / \text { week) in health unit }\end{array}$ & $\begin{array}{l}\text { Health unit } \\
\text { administrative data }\end{array}$ \\
\hline $\begin{array}{l}\text { Population served } \\
\text { (Q11317) }\end{array}$ & Urban/rural/ mixed (municipal designation) & $\begin{array}{l}\text { Health unit } \\
\text { administrative data }\end{array}$ \\
\hline $\begin{array}{l}\text { Functional differentiation } \\
\text { (Q11318) }\end{array}$ & number of divisions & $\begin{array}{l}\text { Health unit } \\
\text { administrative data }\end{array}$ \\
\hline $\begin{array}{l}\text { Complexity } \\
\text { (Q11319) }\end{array}$ & number of programs & $\begin{array}{l}\text { Health un it } \\
\text { administrative data }\end{array}$ \\
\hline $\begin{array}{l}\text { Vertical differentiation } \\
(\mathrm{Q} 11320)\end{array}$ & $\begin{array}{l}\text { Number of levels of workers in organization } \\
\text { (Front line staff to Medical Officer of Health) }\end{array}$ & $\begin{array}{l}\text { Health unit } \\
\text { administrative data }\end{array}$ \\
\hline $\begin{array}{l}\text { Research activity } \\
\text { (Q11322) }\end{array}$ & $\begin{array}{l}\text { Health unit involvement in research activities } \\
\text { Yes/No }\end{array}$ & $\begin{array}{l}\text { Health unit } \\
\text { administrative data }\end{array}$ \\
\hline $\begin{array}{l}\text { Research activity } \\
\text { (Q11321) }\end{array}$ & $\begin{array}{l}\text { Number of research projects health unit is } \\
\text { involved in }\end{array}$ & $\begin{array}{l}\text { Health unit } \\
\text { administrative data }\end{array}$ \\
\hline $\begin{array}{l}\text { Teaching Health Unit } \\
\text { (Q11324) }\end{array}$ & $\begin{array}{l}\text { Designated as a teaching health unit (now } \\
\text { PHRED) }\end{array}$ & $\begin{array}{l}\text { Health unit } \\
\text { administrative data }\end{array}$ \\
\hline $\begin{array}{l}\text { Formalization } \\
\text { (Q21772) }\end{array}$ & $\begin{array}{l}\text { Degree to which organization follows policies } \\
\text { and procedures } \\
5 \text { point Likert scale } \\
1 \text { = strongly agree } \\
5 \text { = strongly disagree }\end{array}$ & $\begin{array}{l}\text { Decision-maker } \\
\text { perceptions }\end{array}$ \\
\hline $\begin{array}{l}\text { Financial (slack) resources } \\
\text { (Q21774) }\end{array}$ & $\begin{array}{l}\text { Perceived barrier to using research evidence } \\
\text { based on limited organizational resources } \\
5 \text { point Likert scale } \\
1 \text { = strongly agree } \\
5 \text { = strongly disagree }\end{array}$ & $\begin{array}{l}\text { Decision-maker } \\
\text { perceptions }\end{array}$ \\
\hline $\begin{array}{l}\text { Organizational Culture } \\
\text { Research value } \\
\text { (Q21773) }\end{array}$ & $\begin{array}{l}\text { The organization values the use of research } \\
5 \text { point Likert scale } \\
\text { I = strongly agree } \\
5 \text { = strongly disagree }\end{array}$ & $\begin{array}{l}\text { Decision-maker } \\
\text { perceptions }\end{array}$ \\
\hline $\begin{array}{l}\text { Staff training } \\
(\mathrm{Q} 21775)\end{array}$ & $\begin{array}{l}\text { Ongoing training for staff in research methods } \\
\text { and critical appraisal } \\
5 \text { point Likert scale } \\
1 \text { = strongly agree } \\
5 \text { = strongly disagree }\end{array}$ & $\begin{array}{l}\text { Decision-maker } \\
\text { perceptions }\end{array}$ \\
\hline $\begin{array}{l}\text { External Communication } \\
(21776)\end{array}$ & $\begin{array}{l}\text { Existence of mechanisms that promote transfer } \\
\text { of new information into organization } \\
5 \text { point Likert scale } \\
1 \text { = strongly agree } \\
5 \text { = strongly disagree }\end{array}$ & $\begin{array}{l}\text { Decision-maker } \\
\text { perceptions }\end{array}$ \\
\hline
\end{tabular}




\begin{tabular}{|c|c|c|}
\hline $\begin{array}{l}\text { Centralization } \\
\text { Information searched for } \\
\text { before making decision } \\
\text { (Q21783) }\end{array}$ & $\begin{array}{l}\text { Extent to which information is routinely } \\
\text { searched for before making decisions } \\
5 \text { point Likert scale } \\
\text { 1) = not at all } \\
\text { 2) = extensively }\end{array}$ & $\begin{array}{l}\text { Decision-maker } \\
\text { perceptions }\end{array}$ \\
\hline $\begin{array}{l}\text { Centralization } \\
\text { Importance of research } \\
\text { evidence to decisions } \\
\text { (Q21786) }\end{array}$ & $\begin{array}{l}\text { Importance routinely placed on research } \\
\text { evidence in decision-making } \\
\text { 1) = not at all } \\
\text { 2) = very }\end{array}$ & $\begin{array}{l}\text { Decision-maker } \\
\text { perceptions }\end{array}$ \\
\hline \multicolumn{3}{|l|}{$\begin{array}{l}\text { Environmental } \\
\text { Characteristics }\end{array}$} \\
\hline $\begin{array}{l}\text { Regulations and legislation } \\
\text { (Q21790) }\end{array}$ & $\begin{array}{l}\text { degree to which provincial and local regulations } \\
\text { and legislation impact on program decisions } \\
5 \text { point Likert scale } \\
1 \text { = strongly agree } \\
5 \text { = strongly disagree }\end{array}$ & $\begin{array}{l}\text { Decision-maker } \\
\text { perceptions }\end{array}$ \\
\hline $\begin{array}{l}\text { Network embeddedness } \\
\text { Q21791) }\end{array}$ & $\begin{array}{l}\text { degree to which decisions are made in } \\
\text { collaboration with other institutions and } \\
\text { agencies } \\
5 \text { point Likert scale } \\
1=\text { strongly agree } \\
5=\text { strongly disagree }\end{array}$ & $\begin{array}{l}\text { Decision-maker } \\
\text { perceptions }\end{array}$ \\
\hline $\begin{array}{l}\text { MOH relationship with board } \\
\text { (Q21792) }\end{array}$ & $\begin{array}{l}\text { Amount of influence the MOH has over } \\
\text { decisions that the local board of health makes } \\
5 \text { point Likert scale } \\
1 \text { = strongly agree } \\
5 \text { = strongly disagree }\end{array}$ & $\begin{array}{l}\text { Decision-maker } \\
\text { perceptions }\end{array}$ \\
\hline $\begin{array}{l}\text { MOH relationship with } \\
\text { politicians } \\
\text { (Q21793) }\end{array}$ & $\begin{array}{l}\text { Amount of influence the MOH has over } \\
\text { decisions that local politicians make } \\
5 \text { point Likert scale } \\
1 \text { = strongly agree } \\
5 \text { = strongly disagree }\end{array}$ & $\begin{array}{l}\text { Decision-maker } \\
\text { perceptions }\end{array}$ \\
\hline $\begin{array}{l}\text { Board influenced by research } \\
\text { (Q21794) }\end{array}$ & $\begin{array}{l}\text { Degree to which local board of health is } \\
\text { influenced by research evidence } \\
5 \text { point Likert scale } \\
1 \text { = strongly agree } \\
5=\text { strongly disagree }\end{array}$ & $\begin{array}{l}\text { Decision-maker } \\
\text { perceptions }\end{array}$ \\
\hline $\begin{array}{l}\text { Politicians influenced by } \\
\text { research } \\
\text { (Q21795) }\end{array}$ & $\begin{array}{l}\text { Degree to which local politicians are influenced } \\
\text { by research evidence } \\
5 \text { point Likert scale } \\
1 \text { = strongly agree }\end{array}$ & $\begin{array}{l}\text { Decision-maker } \\
\text { perceptions }\end{array}$ \\
\hline \multicolumn{3}{|l|}{$\begin{array}{l}\text { Characteristics of } \\
\text { Innovation }\end{array}$} \\
\hline $\begin{array}{l}\text { Relative advantage } \mathrm{Tl} \\
\text { (Before being exposed to } \\
\text { innovation) } \\
((\mathrm{Q} 20765)\end{array}$ & $\begin{array}{l}\text { Priority that should be given to conducting } \\
\text { systematic overviews } \mathrm{Tl} \\
4 \text { point Likert scale } \\
1 \text { = low } \\
4 \text { = top }\end{array}$ & OHCEN data \\
\hline
\end{tabular}




\begin{tabular}{|c|c|c|}
\hline $\begin{array}{l}\text { Relative advantage T2 } \\
\text { (After being exposed to } \\
\text { innovation) } \\
\text { (Q20479) }\end{array}$ & $\begin{array}{l}\text { Priority that should be given to conducting } \\
\text { systematic overviews } T 2 \\
4 \text { point Likert scale } \\
1=\text { low } \\
4 \text { = top }\end{array}$ & OHCEN data \\
\hline $\begin{array}{l}\text { Compatibility } \\
\text { (Q20734) }\end{array}$ & $\begin{array}{l}\text { weight given to research when making decisions } \\
5 \text { point Likert scale } \\
1=0-20 \% \\
5=81-100 \%\end{array}$ & OHCEN data \\
\hline $\begin{array}{l}\text { Compatibility } \\
\text { (Q20735) }\end{array}$ & $\begin{array}{l}\text { weight that should be given to research when } \\
\text { making decisions } \\
5 \text { point Likert scale } \\
\text { l }=0-20 \% \\
5=81-100 \%\end{array}$ & OHCEN data \\
\hline $\begin{array}{l}\text { Compatibility } \\
\text { Appropriateness of timing for } \\
\text { receiving overviews } \\
\text { (Q20446) }\end{array}$ & $\begin{array}{l}\text { How appropriate was the timing for receiving } \\
\text { the systematic overview } \\
4 \text { point Likert scale } \\
1 \text { = excellent } \\
4 \text { = poor }\end{array}$ & $\begin{array}{l}\text { Decision-maker } \\
\text { perceptions }\end{array}$ \\
\hline $\begin{array}{l}\text { Compatibility } \\
\text { Relevance } \\
\text { OHCEN data (Q20447) }\end{array}$ & $\begin{array}{l}\text { How relevant was the systematic overview to } \\
\text { current program decisions } \\
4 \text { point Likert scale } \\
1 \text { = excellent } \\
4=\text { poor }\end{array}$ & $\begin{array}{l}\text { Decision-maker } \\
\text { perceptions }\end{array}$ \\
\hline $\begin{array}{l}\text { Relative Advantage } \\
\text { (Before being exposed to } \\
\text { innovation Tl) } \\
\text { ((Q20749) }\end{array}$ & $\begin{array}{l}\text { Do you think systematic overview's could } \\
\text { overcome barrier of limited access to literature } \\
5 \text { point Likert scale } \\
1=\text { definitely won't } \\
5=\text { definitely will }\end{array}$ & OHCEN data \\
\hline $\begin{array}{l}\text { Relative Advantage Tl } \\
\text { (Before being exposed to } \\
\text { innovation) } \\
\text { (Q20751) }\end{array}$ & $\begin{array}{l}\text { Do you think systematic overviews could } \\
\text { overcome barrier of not having enough time to } \\
\text { review the literature } \\
5 \text { point Likert scale } \\
\text { I = definitely won't } \\
5=\text { definitely will }\end{array}$ & OHCEN data \\
\hline $\begin{array}{l}\text { Relative Advantage } \mathrm{Tl} \\
\text { Before being exposed to } \\
\text { innovation) } \\
\text { (Q20752) }\end{array}$ & $\begin{array}{l}\text { Do you think systematic overviews could } \\
\text { overcome barrier of limited critical appraisal } \\
\text { skills } \\
5 \text { point Likert scale } \\
1 \text { = definitely won't } \\
5=\text { definitely will }\end{array}$ & OHCEN data \\
\hline $\begin{array}{l}\text { Relative Advantage } \mathrm{Tl} \\
\text { Before being exposed to } \\
\text { innovation) } \\
\text { (Q20757) }\end{array}$ & $\begin{array}{l}\text { Do you think systematic overviews could } \\
\text { overcome barrier of limited resources } \\
5 \text { point Likert scale } \\
1 \text { = definitely won't } \\
5=\text { definitely will }\end{array}$ & OHCEN data \\
\hline
\end{tabular}




\begin{tabular}{|c|c|c|}
\hline $\begin{array}{l}\text { Relative Advantage } \mathrm{Tl} \\
\text { Before being exposed to } \\
\text { innovation) } \\
\text { (Q20754) }\end{array}$ & $\begin{array}{l}\text { Do you think systematic overviews could } \\
\text { overcome barrier of research evidence not being } \\
\text { relevant to decision being made } \\
5 \text { point Likert scale } \\
1=\text { definitely won't } \\
5=\text { definitely will }\end{array}$ & OHCEN data \\
\hline $\begin{array}{l}\text { Relative Advantage } \mathrm{Tl} \\
\text { Before being exposed to } \\
\text { innovation) } \\
\text { (Q20755) }\end{array}$ & $\begin{array}{l}\text { Do you think systematic overviews could } \\
\text { overcome barrier of unsupportive work } \\
\text { environments for using research evidence in } \\
\text { decision-making } \\
5 \text { point Likert scale } \\
1=\text { definitely won't } \\
5=\text { definitely will }\end{array}$ & OHCEN data \\
\hline $\begin{array}{l}\text { Relative Advantage Tl } \\
\text { Before being exposed to } \\
\text { innovation) } \\
\text { (Q20756) }\end{array}$ & $\begin{array}{l}\text { Do you think systematic overviews could } \\
\text { overcome barrier of lack of decision-making } \\
\text { authoirty } \\
5 \text { point Likert scale } \\
1=\text { definitely won't } \\
5=\text { definitely will }\end{array}$ & OHCEN data \\
\hline $\begin{array}{l}\text { Relative Advantage T2 } \\
\text { (After being exposed to } \\
\text { innovation) } \\
\text { (Q20459) }\end{array}$ & $\begin{array}{l}\text { Do you think systematic overviews could } \\
\text { overcome barrier of limited access to literature } \\
5 \text { point Likert scale } \\
1 \text { = definitely won't } \\
5 \text { = definitely will } \\
\text { 2) time } \\
\text { 3) critical appraisal skills } \\
\text { 4) limited resources } \\
\text { 5) relevance } \\
\text { 6) unsupportive work environment } \\
\text { 7) authority }\end{array}$ & OHCEN data \\
\hline $\begin{array}{l}\text { Relative Advantage } \mathrm{T} 2 \\
\text { (After being exposed to } \\
\text { innovation) } \\
(\mathrm{Q} 20458)\end{array}$ & $\begin{array}{l}\text { Do you think systematic overviews could } \\
\text { overcome barrier of not having enough time to } \\
\text { review the literature } \\
5 \text { point Likert scale } \\
1=\text { definitely won't } \\
5=\text { definitely will }\end{array}$ & OHCEN data \\
\hline $\begin{array}{l}\text { Relative Advantage T2 } \\
\text { (After being exposed to } \\
\text { innovation) } \\
\text { (Q2046I) }\end{array}$ & $\begin{array}{l}\text { Do you think systematic overviews could } \\
\text { overcome barrier of limited critical appraisal } \\
\text { skills } \\
5 \text { point Likert scale } \\
\text { l = definitely won't } \\
5=\text { definitely will }\end{array}$ & OHCEN data \\
\hline $\begin{array}{l}\text { Relative Advantage T2 } \\
\text { (After being exposed to } \\
\text { innovation) } \\
\text { (Q20466) }\end{array}$ & $\begin{array}{l}\text { Do you think systematic overviews could } \\
\text { overcome barrier of limited financial resources } \\
5 \text { point Likert scale } \\
\text { I = definitely won't } \\
5=\text { definitely will }\end{array}$ & OHCEN data \\
\hline
\end{tabular}




\begin{tabular}{|c|c|c|}
\hline $\begin{array}{l}\text { Relative Advantage } \mathrm{T} 2 \\
\text { (After being exposed to } \\
\text { innovation) } \\
(\mathrm{Q} 20462)\end{array}$ & $\begin{array}{l}\text { Do you think systematic overviews could } \\
\text { overcome barrier of research evidence not being } \\
\text { relevant to decision being made } \\
5 \text { point Likert scale } \\
1=\text { definitely won } t \\
5=\text { definitely will }\end{array}$ & OHCEN data \\
\hline $\begin{array}{l}\text { Relative Advantage T2 } \\
\text { (After being exposed to } \\
\text { innovation) } \\
\text { (Q20464) }\end{array}$ & $\begin{array}{l}\text { Do you think systematic overviews could } \\
\text { overcome barrier of unsupportive work } \\
\text { environments to for using research evidence for } \\
\text { decision-making } \\
5 \text { point Likert scale } \\
1 \text { = definitely won't } \\
5=\text { definitely will }\end{array}$ & OHCEN data \\
\hline $\begin{array}{l}\text { Relative Advantage T2 } \\
\text { (After being exposed to } \\
\text { innovation) } \\
\text { (Q20465) }\end{array}$ & $\begin{array}{l}\text { Do you think systematic overviews could } \\
\text { overcome barrier of lack of decision-making } \\
\text { authority } \\
5 \text { point Likert scale } \\
1=\text { definitely won } t \\
5=\text { definitely will }\end{array}$ & OHCEN data \\
\hline $\begin{array}{l}\text { Complexity } \\
\text { Ease of Use } \\
\text { (Q20447) }\end{array}$ & $\begin{array}{l}\text { How easy was the systematic overview to use } \\
4 \text { point Likert scale } \\
1 \text { = excellent } \\
4=\text { poor }\end{array}$ & $\begin{array}{l}\text { Decision-maker } \\
\text { perceptions }\end{array}$ \\
\hline $\begin{array}{l}\text { Bandwagon effect } \\
(\mathrm{Q} 21815)\end{array}$ & $\begin{array}{l}\text { Perception of the percentage of other health } \\
\text { units using the systematic overviews }\end{array}$ & $\begin{array}{l}\text { Decision-maker } \\
\text { perceptions }\end{array}$ \\
\hline $\begin{array}{l}\text { Bandwagon effect } \\
\text { (Q21816) }\end{array}$ & $\begin{array}{l}\text { Percentage of other colleagues in the same } \\
\text { position using the systematic overviews }\end{array}$ & $\begin{array}{l}\text { Decision-maker } \\
\text { perceptions }\end{array}$ \\
\hline \multicolumn{3}{|l|}{$\begin{array}{l}\text { Individual } \\
\text { Characteristics }\end{array}$} \\
\hline $\begin{array}{l}\text { Baseline Position in } 1996 \\
\text { (typ) }\end{array}$ & Respondent's current position & OHCEN data \\
\hline $\begin{array}{l}\text { Tenure } \\
\text { (Q20788) }\end{array}$ & Years in current position & OHCEN data \\
\hline $\begin{array}{l}\text { Tenure } \\
\text { (Q20789) }\end{array}$ & Years in public health & OHCEN data \\
\hline $\begin{array}{l}\text { Education } \\
\text { (Q20792) }\end{array}$ & 1) Years since graduation & OHCEN data \\
\hline $\begin{array}{l}\text { Age } \\
\text { (Q20793) }\end{array}$ & 10 year age categories & OHCEN data \\
\hline $\begin{array}{l}\text { Consultant contact } \\
\text { (Q20705) }\end{array}$ & Access to research consultant (Yes/No) & OHCEN data \\
\hline $\begin{array}{l}\text { Access to on-line searching } \\
\text { (Q20709) }\end{array}$ & $\begin{array}{l}\text { Direct access in organization to on-line database } \\
\text { searching (Yes/No) }\end{array}$ & OHCEN data \\
\hline $\begin{array}{l}\text { Number of articles retrieved } \\
\text { in past month } \\
\text { (Q20707) }\end{array}$ & $\begin{array}{l}\text { 1) None } \\
2<10 \\
310-19 \\
420-29\end{array}$ & OHCEN data \\
\hline
\end{tabular}




\begin{tabular}{|c|c|c|}
\hline & $5>30$ & \\
\hline $\begin{array}{l}\text { Percentage of retrieved } \\
\text { articles read on in past month } \\
\text { (Q20708) }\end{array}$ & $\begin{array}{l}1 \text { None } \\
2<25 \\
325-50 \\
451-75 \\
5>75 \%\end{array}$ & OHCEN data \\
\hline $\begin{array}{l}\text { Cosmopolitanism } \\
(Q 20715)\end{array}$ & $\begin{array}{l}\text { Days at external meetings/conferences in past } \\
\text { year }\end{array}$ & $\overline{\mathrm{OHCEN}}$ data \\
\hline $\begin{array}{l}\text { Authority } \\
\text { (Q20726) }\end{array}$ & $\begin{array}{l}\text { Perceived barrier to using research evidence } \\
\text { based on level of authority to make program } \\
\text { decisions } \\
5 \text { point Likert Scale } \\
1=\text { not a barrier } \\
5=\text { very serious barrier }\end{array}$ & OHCEN data \\
\hline $\begin{array}{l}\text { Critical appraisal } \\
\text { (Q20722) }\end{array}$ & $\begin{array}{l}\text { Perceived barrier to using research evidence } \\
\text { based on critical appraisal skills } \\
5 \text { point Likert Scale } \\
\text { l= not a barrier } \\
5=\text { very serious barrier }\end{array}$ & OHCEN data \\
\hline $\begin{array}{l}\text { Financial resources } \\
\text { (Q20727) }\end{array}$ & $\begin{array}{l}\text { Perceived barrier to using research evidence } \\
\text { based on limited financial resources } \\
5 \text { point Likert Scale } \\
I=\text { not a barrier } \\
5=\text { very sericus barrier }\end{array}$ & OHCEN data \\
\hline $\begin{array}{l}\text { Prior use of systematic } \\
\text { overviews } \\
\text { (Q20745) }\end{array}$ & $\begin{array}{l}\text { Have used systematic overviews in the past to } \\
\text { make program decisions }\end{array}$ & OHCEN data \\
\hline $\begin{array}{l}\text { Future Use } \\
\text { (Q20428) }\end{array}$ & $\begin{array}{l}\text { Expectation of using the systematic overview in } \\
\text { future }\end{array}$ & OHCEN data \\
\hline \multicolumn{3}{|l|}{$\begin{array}{l}\text { Research Utilization } \\
\text { Dependent Variables }\end{array}$} \\
\hline $\begin{array}{l}\text { Use of systematic overview } \\
(\mathrm{Q} 21819)\end{array}$ & $\begin{array}{l}\text { Has the systematic overview been used in the } \\
\text { past two years to make a program decisions }\end{array}$ & $\begin{array}{l}\text { Decision-maker } \\
\text { perceptions }\end{array}$ \\
\hline $\begin{array}{l}\text { Influence on Program } \\
\text { Planning } \\
\text { (Q21806) }\end{array}$ & $\begin{array}{l}\text { Five point Likert Scale } \\
\text { I Not at all } \\
5 \text { A Great Deal }\end{array}$ & $\begin{array}{l}\text { Decision-maker } \\
\text { perceptions }\end{array}$ \\
\hline $\begin{array}{l}\text { Influence on Program } \\
\text { Justification } \\
\text { (Q21807) }\end{array}$ & $\begin{array}{l}\text { Five point Likert Scale } \\
1 \text { Not at all } \\
5 \text { A Great Deal }\end{array}$ & $\begin{array}{l}\text { Decision-maker } \\
\text { perceptions }\end{array}$ \\
\hline $\begin{array}{l}\text { Influence on Program } \\
\text { Evaluation } \\
(\mathrm{Q} 21808)\end{array}$ & $\begin{array}{l}\text { Five point Likert Scale } \\
1 \text { Not at all } \\
5 \text { A Great Deal }\end{array}$ & $\begin{array}{l}\text { Decision-maker } \\
\text { perceptions }\end{array}$ \\
\hline $\begin{array}{l}\text { Influence on Policy } \\
\text { Development } \\
\text { (Q21809) }\end{array}$ & $\begin{array}{l}\text { Five point Likert Scale } \\
\text { I Not at all } \\
5 \text { A Great Deal }\end{array}$ & $\begin{array}{l}\text { Decision-maker } \\
\text { perceptions }\end{array}$ \\
\hline $\begin{array}{l}\text { Influence on Staff } \\
\text { Development } \\
\text { (Q21810) }\end{array}$ & $\begin{array}{l}\text { Five point Likert Scale } \\
\text { I Not at all } \\
5 \mathrm{~A} \text { Great Deal }\end{array}$ & $\begin{array}{l}\text { Decision-maker } \\
\text { perceptions }\end{array}$ \\
\hline
\end{tabular}


Table 2: Results of the Principal Component Analysis

Rotated Component Matrik

\begin{tabular}{|c|c|c|c|c|c|}
\hline & \multicolumn{5}{|c|}{ Component } \\
\hline & 1 & 2 & 3 & 4 & 5 \\
\hline Q20755 & .710 & $5.621 E-02$ & $9.042 E-02$ & $-2.66 E-02$ & $1.172 E-02$ \\
\hline Q20756 & .688 & -.170 & .203 & $1.289 E-02$ & .104 \\
\hline Q20757 & .655 & $-3.81 E-03$ & .149 & 5.682E-02 & $-8.35 E-02$ \\
\hline Q20754 & .539 & .199 & .220 & 4.376E-02 & .107 \\
\hline Q20734 & .526 & -.165 & -.398 & .373 & -.149 \\
\hline Q20458 & 5.737E-02 & .721 & -.126 & .172 & 7.271E-02 \\
\hline Q20447 & -.193 & -.692 & -.265 & .146 & 7.243E-02 \\
\hline Q20459 & $-9.40 E-02$ & .677 & -.104 & $9.001 E-02$ & $-2.27 E-02$ \\
\hline Q20461 & -.193 & .612 & .244 & .102 & 9.393E-02 \\
\hline Q20445 & -.125 & -.599 & -.231 & -.152 & .392 \\
\hline Q20466 & .270 & -.111 & .695 & $3.582 E-02$ & .123 \\
\hline Q20465 & .370 & $-1.24 E-02$ & .586 & .305 & $-4.60 E-02$ \\
\hline Q20735 & .402 & $6.078 E-02$ & -.517 & .330 & -.105 \\
\hline Q20462 & 6.579E-02 & .217 & .513 & .205 & -.206 \\
\hline Q20464 & .192 & $1.133 E-02$ & .467 & .178 & -.147 \\
\hline Q20479 & $-5.11 E-02$ & -.205 & -.461 & $-3.40 E-02$ & -.245 \\
\hline Q21815 & $4.374 E-02$ & 8.298E-02 & .120 & .853 & 7.681E-02 \\
\hline Q21816 & $-3.91 E-02$ & .121 & .229 & .820 & $-4.62 E-02$ \\
\hline Q20765 & .187 & .262 & 4.662E-02 & .427 & .283 \\
\hline Q20446 & -.302 & -.272 & -.215 & .123 & .565 \\
\hline Q20427 & $-3.05 E-02$ & $-9.70 \mathrm{E}-02$ & .138 & $-3.63 E-02$ & .493 \\
\hline Q20749 & .426 & .406 & 6.631E-02 & 4.963E-02 & .488 \\
\hline Q20751 & .430 & .430 & -.167 & .131 & .437 \\
\hline Q20752 & .249 & .227 & $-2.55 E-02$ & 7.518E-02 & 399 \\
\hline
\end{tabular}

Extraction Method: Principal Component Analysis.

Rotation Method: Varimax with Kaiser Normalization.

a. Rotation converged in $\mathbf{1 5}$ iterations. 
Table 3: Descriptive Statistics:

\begin{tabular}{|c|c|c|c|c|c|}
\hline Variable Label & Operational Definition & Mean/\% & $\begin{array}{l}\text { Standard } \\
\text { Deviation }\end{array}$ & $\mathbf{N}$ & $\begin{array}{l}95 \% \mathrm{CI} \\
(\mathrm{MEAN})\end{array}$ \\
\hline \multicolumn{6}{|l|}{$\begin{array}{l}\text { Organizational } \\
\text { Characteristics }\end{array}$} \\
\hline Size & $\begin{array}{l}\text { Number full time equivalent } \\
\text { employees in health unit }\end{array}$ & 131 & 86.1 & 141 & $115-147$ \\
\hline Population served & Urban/rural/ mixed & $\begin{aligned} \text { Urban } & =51.1 \% \\
\text { Rural } & =29.0 \% \\
\text { Mixed } & =19.9 \%\end{aligned}$ & 0.8 & 141 & NA \\
\hline Complexity & Number of divisions & 4.0 & 1.2 & 141 & $3.83-4.2$ \\
\hline Complexity & Number of program & 25.2 & 9.2 & 141 & $\begin{array}{l}23.68- \\
26.72\end{array}$ \\
\hline $\begin{array}{l}\text { Functional } \\
\text { differentiation }\end{array}$ & $\begin{array}{l}\text { Number of levels of workers } \\
\text { in organization }\end{array}$ & 3.9 & 0.6 & 141 & $\begin{array}{l}3.86- \\
4.07 \\
\end{array}$ \\
\hline Research activity & $\begin{array}{l}\text { 1) Health unit involvement } \\
\text { in research activities }\end{array}$ & $\begin{array}{l}\text { Yes }=91.5 \% \\
\text { No }=8.5 \%\end{array}$ & & 141 & NA \\
\hline Research activity & $\begin{array}{l}\text { Number of research projects } \\
\text { health unit is involved in }\end{array}$ & 6.4 & 3.1 & 141 & $\begin{array}{l}5.87- \\
6.95 \\
\end{array}$ \\
\hline $\begin{array}{l}\text { Teaching Heaith } \\
\text { Unit }\end{array}$ & $\begin{array}{l}\text { Designated as a teaching } \\
\text { health unit (now PHRED) }\end{array}$ & $\begin{array}{l}\text { Yes }=32.6 \% \\
\text { No }=67.4 \%\end{array}$ & & 141 & NA \\
\hline Formalization & $\begin{array}{l}\text { Degree to which } \\
\text { organization follows policies } \\
\text { and procedures } \\
5 \text { point Likert scale } \\
1 \text { = strongly agree } \\
5=\text { strongly disagree }\end{array}$ & $\begin{array}{l}\text { 1) } 42.6 \\
\text { 2) } 52.5 \\
\text { 3) } 2.1 \\
\text { 4) } 2.1 \\
\text { 5) } .7 \\
\text { Mean }=1.66\end{array}$ & 0.695 & 141 & $\begin{array}{l}1.54- \\
1.76\end{array}$ \\
\hline Financial resources & $\begin{array}{l}\text { Perceived barrier to using } \\
\text { research evidence based on } \\
\text { limited organizational } \\
\text { resources } \\
5 \text { point Likert scale } \\
1 \text { = strongly agree } \\
5=\text { strongly disagree }\end{array}$ & $\begin{array}{l}\text { 1) } 14.9 \\
\text { 2) } 50.4 \\
\text { 3) } 17.7 \\
\text { 4) } 13.5 \\
\text { 5) } 3.5 \\
\text { Mean }=2.40\end{array}$ & 1.01 & 141 & $\begin{array}{l}2.24- \\
2.57\end{array}$ \\
\hline Research value & $\begin{array}{l}\text { The organization values the } \\
\text { use of research } \\
5 \text { point Likert scale } \\
1 \text { = strongly agree } \\
5 \text { = strongly disagree }\end{array}$ & $\begin{array}{l}\text { 1) } 41.8 \\
\text { 2) } 48.2 \\
\text { 3) } 5.0 \\
\text { 4) } 5.0 \\
\text { 5) } 0.0 \\
\text { Mean }=1.73\end{array}$ & 0.77 & 141 & $\begin{array}{l}1.60- \\
1.86\end{array}$ \\
\hline
\end{tabular}




\begin{tabular}{|c|c|c|c|c|c|}
\hline Staff training & $\begin{array}{l}\text { ongoing training for staff in } \\
\text { research methods and critical } \\
\text { appraisal } \\
5 \text { point Likert scale } \\
1=\text { strongly agree } \\
5=\text { strongly disagree }\end{array}$ & $\begin{array}{l}\text { 1) } 12.1 \\
\text { 2) } 37.6 \\
\text { 3) } 22.0 \\
\text { 4) } 23.4 \\
\text { 5) } 5.0 \\
\text { Mean }=2.72\end{array}$ & 1.1 & 141 & $\begin{array}{l}2.53- \\
2.90\end{array}$ \\
\hline $\begin{array}{l}\text { Integrative } \\
\text { mechanisms }\end{array}$ & $\begin{array}{l}\text { existence of mechanisms that } \\
\text { promote transfer of new } \\
\text { information into organization } \\
5 \text { point Likert scale } \\
1 \text { = strongly agree } \\
5=\text { strongly disagree }\end{array}$ & $\begin{array}{l}\text { 1) } 24.8 \\
\text { 2) } 36.9 \\
\text { 3) } 15.6 \\
\text { 4) } 14.9 \\
\text { 5) } 7.8 \\
\text { Mean }=2.44\end{array}$ & 1.23 & 141 & $\begin{array}{l}2.23- \\
2.64\end{array}$ \\
\hline $\begin{array}{l}\text { Extent information } \\
\text { searched for }\end{array}$ & $\begin{array}{l}\text { Extent to which information } \\
\text { is routinely searched for } \\
\text { beofre making decisions } \\
\text { 1) = not at all } \\
\text { 5) = extensively }\end{array}$ & $\begin{array}{l}\text { 1) } 2.9 \\
\text { 2) } 3.6 \\
\text { 3) } 25.7 \\
\text { 4) } 20.0 \\
\text { 5) } 47.9 \\
\text { Mean }=4.06\end{array}$ & 1.07 & 140 & $\begin{array}{l}3.89- \\
4.24\end{array}$ \\
\hline $\begin{array}{l}\text { Importance of } \\
\text { research evidence }\end{array}$ & $\begin{array}{l}\text { Importance routinely placed } \\
\text { on research evidence to make } \\
\text { decisions } \\
\text { 1) = not at all } \\
5)=\text { very }\end{array}$ & $\begin{array}{l}\text { 1) } 19.3 \\
\text { 2) } 4.3 \\
\text { 3) } 31.4 \\
\text { 4) } 12.9 \\
\text { 5) } 32.1 \\
\text { Mean }=3.34 \\
\end{array}$ & 1.46 & 140 & $\begin{array}{l}3.10- \\
3.59\end{array}$ \\
\hline \multicolumn{6}{|l|}{$\begin{array}{l}\text { Environmental } \\
\text { Characteristics }\end{array}$} \\
\hline $\begin{array}{l}\text { Regulations and } \\
\text { legislation }\end{array}$ & $\begin{array}{l}\text { degree to which provincial } \\
\text { and local regulations and } \\
\text { legislation impact on } \\
\text { program decisions } \\
5 \text { point Likert scale } \\
1=\text { strongly agree } \\
5=\text { strongly disagree }\end{array}$ & $\begin{array}{l}\text { 1) } 59.6 \\
\text { 2) } 34.8 \\
\text { 3) } 4.3 \\
\text { 4) } 1.4 \\
\text { 5) } 0.0 \\
\text { Mean = } 1.48\end{array}$ & 0.65 & 141 & $\begin{array}{l}1.37 \\
1.58\end{array}$ \\
\hline $\begin{array}{l}\text { Network } \\
\text { embeddedness }\end{array}$ & $\begin{array}{l}\text { degree to which decisions are } \\
\text { made in collaboration with } \\
\text { other institutions and } \\
\text { agencies } \\
5 \text { point Likert scale } \\
1=\text { strongly agree } \\
5=\text { strongly disagree }\end{array}$ & $\begin{array}{l}\text { 1) } 22.0 \\
\text { 2) } 48.9 \\
\text { 3) } 17.0 \\
\text { 4) } 12.1 \\
\text { 5) } 0.0 \\
\text { Mean }=2.19\end{array}$ & 0.92 & 141 & $\begin{array}{l}2.04- \\
2.34\end{array}$ \\
\hline
\end{tabular}




\begin{tabular}{|c|c|c|c|c|c|}
\hline $\begin{array}{l}\text { MOH relationship } \\
\text { with board }\end{array}$ & $\begin{array}{l}\text { amount of influence the } \\
\text { MOH has over decisions that } \\
\text { the local board of health } \\
\text { makes } \\
5 \text { point Likert scale } \\
1=\text { strongly agree } \\
5=\text { strongly disagree }\end{array}$ & $\begin{array}{l}\text { 1) } 48.2 \\
\text { 2) } 35.5 \\
\text { 3) } 8.5 \\
\text { 4) } 5.0 \\
\text { 5) } 0.7 \\
\text { Mean }=1.72\end{array}$ & 0.88 & 138 & $\begin{array}{l}1.57- \\
1.87\end{array}$ \\
\hline $\begin{array}{l}\text { MOH relationship } \\
\text { with politicians }\end{array}$ & $\begin{array}{l}\text { amount of influence the } \\
\text { MOH has over decisions that } \\
\text { local politicians make } \\
5 \text { point Likert scale } \\
1=\text { strongly agree } \\
5=\text { strongly disagree }\end{array}$ & $\begin{array}{l}\text { 1) } 9.2 \\
\text { 2) } 33.3 \\
\text { 3) } 27.7 \\
\text { 4) } 19.1 \\
\text { 5) } 6.4 \\
\text { Mean }=2.79 \\
\end{array}$ & 1.08 & 135 & $\begin{array}{l}2.61- \\
2.98\end{array}$ \\
\hline $\begin{array}{l}\text { Board influenced by } \\
\text { research }\end{array}$ & $\begin{array}{l}\text { Degree to which local board } \\
\text { of health is influenced by } \\
\text { research evidence } \\
5 \text { point Likert scale } \\
1=\text { strongly agree } \\
5=\text { strongly disagree }\end{array}$ & $\begin{array}{l}\text { 1) } 7.4 \\
\text { 2) } 25.2 \\
\text { 3) } 28.1 \\
\text { 4) } 27.4 \\
\text { 5) } 11.9 \\
\text { Mean }=3.11 \\
\end{array}$ & 1.14 & 135 & $\begin{array}{l}2.92- \\
3.30\end{array}$ \\
\hline $\begin{array}{l}\text { Politicians } \\
\text { influenced by } \\
\text { research }\end{array}$ & $\begin{array}{l}\text { Degree to which local } \\
\text { politicians are influenced by } \\
\text { research evidence } \\
5 \text { point Likert scale } \\
1 \text { = strongly agree }\end{array}$ & $\begin{array}{l}\text { 1) } 2.3 \\
\text { 2) } 13.5 \\
\text { 3) } 28.6 \\
\text { 4) } 33.8 \\
\text { 5) } 21.8 \\
\text { Mean }=3.59\end{array}$ & 1.04 & 133 & $\begin{array}{l}3.41- \\
3.77\end{array}$ \\
\hline
\end{tabular}




\begin{tabular}{|c|c|c|c|c|c|}
\hline $\begin{array}{l}\text { Characteristics of } \\
\text { Innovation }\end{array}$ & & & & & \\
\hline $\begin{array}{l}\text { Relative advantage } \\
\mathrm{Tl} \\
\text { (Before being } \\
\text { exposed to } \\
\text { innovation) }\end{array}$ & $\begin{array}{l}\text { Priority that should be given } \\
\text { to conducting systematic } \\
\text { overviews } \\
4 \text { point Likert scale } \\
\text { 1) low } \\
\text { 2) middle } \\
\text { 3) high } \\
\text { 4) top }\end{array}$ & $\begin{array}{l}\text { 1) } 2.1 \\
\text { 2) } 22.7 \\
\text { 3) } 67.4 \\
\text { 4) } 7.8 \\
\text { Mean }=3.81\end{array}$ & 0.60 & 141 & $\begin{array}{l}3.71- \\
3.91\end{array}$ \\
\hline $\begin{array}{l}\text { Relative advantage } \\
\text { T2 } \\
\text { (After being } \\
\text { exposed to } \\
\text { innovation) }\end{array}$ & $\begin{array}{l}\text { Priority that should be given } \\
\text { to conducting systematic } \\
\text { overviews } \\
4 \text { point Likert scale } \\
\text { 1) low } \\
\text { 2) middle } \\
\text { 3) high } \\
\text { 4) top } \\
\end{array}$ & $\begin{array}{l}\text { 1) } 0.0 \\
\text { 2) } 12.3 \\
\text { 3) } 75.4 \\
\text { 4) } 12.3 \\
\text { Mean }=2.0\end{array}$ & 0.5 & 138 & $\begin{array}{l}1.92- \\
2.08\end{array}$ \\
\hline Compatibility & $\begin{array}{l}\text { weight given to research } \\
\text { when making decisions } \\
\text { 5 point Likert scale } \\
\text { 1) } 0-20 \% \\
\text { 2) } 21-40 \% \\
\text { 3) } 41-60 \% \\
\text { 4) } 61-80 \% \\
\text { 5) } 81-100 \% \\
\end{array}$ & $\begin{array}{lr}\text { 1) } & 18.4 \\
\text { 2) } & 30.5 \\
\text { 3) } & 29.1 \\
\text { 4) } & 17.0 \\
\text { 5) } & 5.0 \\
2.6 & \end{array}$ & 1.12 & 141 & $\begin{array}{l}2.41- \\
2.78\end{array}$ \\
\hline Compatibility & $\begin{array}{l}\text { weight that should be given } \\
\text { to research when making } \\
\text { decisions } \\
\text { 5 point Likert scale } \\
\text { 1) } 0-20 \% \\
\text { 2) } 21-40 \% \\
\text { 3) } 41-60 \% \\
\text { 4) } 61-80 \% \\
\text { 5) } 81-100 \% \\
\end{array}$ & $\begin{array}{l}\text { 1) } 1.4 \\
\text { 2) } 7.1 \\
\text { 3) } 29.8 \\
\text { 4) } 42.6 \\
\text { 5) } 19.1 \\
\text { Mean }=3.71\end{array}$ & 0.91 & 141 & $\begin{array}{l}3.56- \\
3.86\end{array}$ \\
\hline $\begin{array}{l}\text { Compatibility } \\
\text { Timing }\end{array}$ & $\begin{array}{l}\text { How appropriate was the } \\
\text { timing for receiving the } \\
\text { systematic overview } \\
4 \text { point Likert scale } \\
1=\text { excellent } \\
4=\text { poor }\end{array}$ & $\begin{array}{l}\text { 1) } 45.6 \\
\text { 2) } 35.0 \\
\text { 3) } 8.7 \\
\text { 4) } 8.7 \\
\text { Mean }=1.86\end{array}$ & 1.03 & 103 & $\begin{array}{l}1.66- \\
2.07\end{array}$ \\
\hline
\end{tabular}




\begin{tabular}{|c|c|c|c|c|c|}
\hline $\begin{array}{l}\text { Compatibility } \\
\text { Relevance }\end{array}$ & $\begin{array}{l}\text { How relevant was the } \\
\text { systematic overview to } \\
\text { current program decisions } \\
4 \text { point Likert scale } \\
1=\text { excellent } \\
4=\text { poor }\end{array}$ & $\begin{array}{l}\text { 1) } 37.4 \\
\text { 2) } 52.5 \\
\text { 3) } 7.1 \\
\text { 4) } 3.0 \\
\text { Mean =1.76 }\end{array}$ & .72 & 99 & $\begin{array}{l}1.61- \\
1.90\end{array}$ \\
\hline $\begin{array}{l}\text { Relative Advantage } \\
\mathrm{T} \text { l } \\
\text { (Before being } \\
\text { exposed to } \\
\text { innovation) }\end{array}$ & $\begin{array}{l}\text { 1) Do you think systematic } \\
\text { overviews could overcome } \\
\text { barrier of limited access to } \\
\text { literature } \\
5 \text { point Likert scale } \\
1=\text { definitely won't } \\
5=\text { definitely will }\end{array}$ & $\begin{array}{l}\text { 1) } 2.1 \\
\text { 2) } 14.2 \\
\text { 3) } 12.8 \\
\text { 4) } 36.2 \\
\text { 5) } 34.0 \\
\text { Mean }=3.88\end{array}$ & 1.12 & 141 & $\begin{array}{l}3.68 \\
4.05\end{array}$ \\
\hline $\begin{array}{l}\text { Relative Advantage } \\
\text { T I } \\
\text { (Before being } \\
\text { exposed to } \\
\text { innovation) }\end{array}$ & $\begin{array}{l}\text { Do you think systematic } \\
\text { overviews could overcome } \\
\text { barrier of not having enough } \\
\text { time to review the literature } \\
5 \text { point Likert scale } \\
1=\text { definitely won't } \\
5=\text { definitely will }\end{array}$ & $\begin{array}{l}\text { 1) } 2.8 \\
\text { 2) } 4.3 \\
\text { 3) } 7.1 \\
\text { 4) } 24.8 \\
\text { 5) } 60.3 \\
\text { Mean }=4.38\end{array}$ & 1.00 & 141 & $\begin{array}{l}4.20- \\
4.53\end{array}$ \\
\hline $\begin{array}{l}\text { Relative Advantage } \\
\text { Tl } \\
\text { (Before being } \\
\text { exposed to } \\
\text { innovation) }\end{array}$ & $\begin{array}{l}\text { Do you think systematic } \\
\text { overviews could overcome } \\
\text { barrier of limited critical } \\
\text { appraisal skills } \\
5 \text { point Likert scale } \\
1=\text { definitely won't } \\
5=\text { definitely will }\end{array}$ & $\begin{array}{l}\text { 1) } 0.0 \\
\text { 2) } 2.1 \\
\text { 3) } 13.5 \\
\text { 4) } 31.9 \\
\text { 5) } 51.8 \\
\text { Mean }=4.35\end{array}$ & 0.80 & 141 & $\begin{array}{l}4.21- \\
4.48\end{array}$ \\
\hline $\begin{array}{l}\text { Relative Advantage } \\
\mathrm{T} \text { l } \\
\text { (Before being } \\
\text { exposed to } \\
\text { innovation) }\end{array}$ & $\begin{array}{l}\text { Do you think systematic } \\
\text { overviews could overcome } \\
\text { barrier of limited financial } \\
\text { resources } \\
5 \text { point Likert scale } \\
1=\text { definitely won't } \\
5=\text { definitely will }\end{array}$ & $\begin{array}{l}\text { 1) } 9.9 \\
\text { 2) } 44.0 \\
\text { 3) } 21.3 \\
\text { 4) } 17.0 \\
\text { 5) } 7.1 \\
\text { Mean }=2.70\end{array}$ & 1.13 & 141 & $\begin{array}{l}2.49- \\
2.85\end{array}$ \\
\hline $\begin{array}{l}\text { Relative Advantage } \\
\text { Tl } \\
\text { (Before being } \\
\text { exposed to } \\
\text { innovation) }\end{array}$ & $\begin{array}{l}\text { Do you think systematic } \\
\text { overviews could overcome } \\
\text { barrier research evidence not } \\
\text { being relevant to decision } \\
\text { being made } \\
5 \text { point Likert scale } \\
1 \text { = definitely won't } \\
5 \text { = definitely will }\end{array}$ & $\begin{array}{l}\text { 1) } 2.1 \\
\text { 2) } 18.4 \\
\text { 3) } 29.8 \\
\text { 4) } 21.3 \\
\text { 5) } 27.0 \\
\text { Mean }=3.57\end{array}$ & 1.17 & 141 & $\begin{array}{l}3.34- \\
3.72\end{array}$ \\
\hline
\end{tabular}




\begin{tabular}{|c|c|c|c|c|c|}
\hline $\begin{array}{l}\text { Relative Advantage } \\
\text { Tl } \\
\text { (Before being } \\
\text { exposed to } \\
\text { innovation) }\end{array}$ & $\begin{array}{l}\text { Do you think systematic } \\
\text { overviews could overcome } \\
\text { barrier of unsupportive work } \\
\text { environments for using } \\
\text { research evidence for } \\
\text { decision-making } \\
5 \text { point Likert scale } \\
1=\text { definitely won't } \\
5 \text { = definitely will }\end{array}$ & $\begin{array}{l}\text { 1) } 5.0 \\
\text { 2) } 30.5 \\
\text { 3) } 24.8 \\
\text { 4) } 26.2 \\
\text { 5) } 12.8 \\
\text { Mean = 3.13 }\end{array}$ & 1.15 & 141 & $\begin{array}{l}2.93- \\
3.30\end{array}$ \\
\hline $\begin{array}{l}\text { Relative Advantage } \\
\text { Tl } \\
\text { (Before being } \\
\text { exposed to } \\
\text { innovation) }\end{array}$ & $\begin{array}{l}\text { Do you think systematic } \\
\text { overviews could overcome } \\
\text { barrier of lack of decision- } \\
\text { making authority } \\
5 \text { point Likert scale } \\
1=\text { definitely won't } \\
5=\text { definitely will }\end{array}$ & $\begin{array}{l}\text { 1) } 7.1 \\
\text { 2) } 31.9 \\
\text { 3) } 27.0 \\
\text { 4) } 24.8 \\
\text { 5) } 8.5 \\
\text { Mean = 2.98 }\end{array}$ & 1.12 & 141 & $\begin{array}{l}2.77- \\
3.14\end{array}$ \\
\hline $\begin{array}{l}\text { Relative Advantage } \\
\text { T2 } \\
\text { (After being } \\
\text { exposed to } \\
\text { innovation) }\end{array}$ & $\begin{array}{l}\text { 1) Do you think systematic } \\
\text { overviews could overcome } \\
\text { barrier of limited access to } \\
\text { literature } \\
5 \text { point Likert scale } \\
1=\text { definitely won't } \\
5=\text { definitely will }\end{array}$ & $\begin{array}{l}\text { 1) } 0.0 \\
\text { 2) } 1.7 \\
\text { 3) } 7.5 \\
\text { 4) } 38.3 \\
\text { 5) } 52.5 \\
\text { Mean }=4.42\end{array}$ & 0.71 & 120 & $\begin{array}{l}4.29- \\
4.54\end{array}$ \\
\hline $\begin{array}{l}\text { Relative Advantage } \\
\text { T2 } \\
\text { (After being } \\
\text { exposed to } \\
\text { innovation) }\end{array}$ & $\begin{array}{l}\text { Do you think systematic } \\
\text { overviews could overcome } \\
\text { barrier of not having enough } \\
\text { time to review the literature } \\
5 \text { point Likert scale } \\
1=\text { definitely won't } \\
5=\text { definitely will }\end{array}$ & $\begin{array}{ll}\text { 1) } & 0.8 \\
\text { 2) } & 0.8 \\
\text { 3) } 5.0 \\
\text { 4) } 27.5 \\
\text { 5) } 65.8 \\
\text { Mean }=4.57\end{array}$ & 0.71 & 120 & $\begin{array}{l}4.44- \\
4.69\end{array}$ \\
\hline $\begin{array}{l}\text { Relative Advantage } \\
\text { T2 } \\
\text { (After being } \\
\text { exposed to } \\
\text { innovation) }\end{array}$ & $\begin{array}{l}\text { Do you think systematic } \\
\text { overviews could overcome } \\
\text { barrier of limited critical } \\
\text { appraisal skills } \\
5 \text { point Likert scale } \\
1=\text { definitely won't } \\
5=\text { definitely will }\end{array}$ & $\begin{array}{l}\text { 1) } 0.0 \\
\text { 2) } 0.8 \\
\text { 3) } 16.0 \\
\text { 4) } 31.1 \\
\text { 5) } 52.0 \\
\text { Mean }=4.34\end{array}$ & 0.78 & 120 & $\begin{array}{l}4.20- \\
4.49\end{array}$ \\
\hline $\begin{array}{l}\text { Relative Advantage } \\
\text { T2 } \\
\text { (After being } \\
\text { exposed to } \\
\text { innovation) }\end{array}$ & $\begin{array}{l}\text { Do you think systematic } \\
\text { overviews could overcome } \\
\text { barrier of limited financial } \\
\text { resources } \\
5 \text { point Likert scale } \\
1=\text { definitely won't } \\
5=\text { definitely will }\end{array}$ & $\begin{array}{l}\text { 1) } 3.3 \\
\text { 2) } 20.0 \\
\text { 3) } 29.2 \\
\text { 4) } 27.5 \\
\text { 5) } 20.0 \\
\text { Mean = 3.41 }\end{array}$ & 1.12 & 120 & $\begin{array}{l}3.21- \\
3.61\end{array}$ \\
\hline
\end{tabular}




\begin{tabular}{|c|c|c|c|c|c|}
\hline $\begin{array}{l}\text { Relative Advantage } \\
\text { T2 } \\
\text { (After being } \\
\text { exposed to } \\
\text { innovation) }\end{array}$ & $\begin{array}{l}\text { Do you think systematic } \\
\text { overviews could overcome } \\
\text { barrier of research not being } \\
\text { relevant to decision being } \\
\text { made } \\
5 \text { point Likert scale } \\
1=\text { definitely won't } \\
5=\text { definitely will }\end{array}$ & $\begin{array}{l}\text { 1) } 0.0 \\
\text { 2) } 10.9 \\
\text { 3) } 30.3 \\
\text { 4) } 44.5 \\
\text { 5) } 14.3 \\
\text { Mean }=3.62\end{array}$ & $\overline{0.86}$ & 119 & $\begin{array}{l}3.47- \\
3.78\end{array}$ \\
\hline $\begin{array}{l}\text { Relative Advantage } \\
\text { T2 } \\
\text { (After being } \\
\text { exposed to } \\
\text { innovation) }\end{array}$ & $\begin{array}{l}\text { Do you think systematic } \\
\text { overviews could overcome } \\
\text { barrier of unsupportive work } \\
\text { environments for using } \\
\text { research evidence for } \\
\text { decision-making } \\
5 \text { point Likert scale } \\
1 \text { = definitely won't } \\
5=\text { definitely will }\end{array}$ & $\begin{array}{l}\text { 1) } 1.7 \\
\text { 2) } 11.0 \\
\text { 3) } 28.8 \\
\text { 4) } 35.6 \\
\text { 5) } 22.9 \\
\text { Mean }=3.67\end{array}$ & 1.0 & 118 & $\begin{array}{l}3.49- \\
3.85\end{array}$ \\
\hline $\begin{array}{l}\text { Relative Advantage } \\
\mathrm{T} 2 \\
\text { (After being } \\
\text { exposed to } \\
\text { innovation) }\end{array}$ & $\begin{array}{l}\text { Do you think systematic } \\
\text { overviews could overcome } \\
\text { barrier of lack of decision- } \\
\text { making authoirty } \\
5 \text { point Likert scale } \\
\text { I = definitely won't } \\
5=\text { definitely will } \\
\end{array}$ & $\begin{array}{l}\text { 1) } 0.0 \\
\text { 2) } 7.6 \\
\text { 3) } 32.8 \\
\text { 4) } 43.7 \\
\text { 5) } 16.0 \\
\text { Mean }=3.68\end{array}$ & 0.83 & 119 & $\begin{array}{l}3.53- \\
3.83\end{array}$ \\
\hline $\begin{array}{l}\text { Complexity } \\
\text { Ease of Use }\end{array}$ & $\begin{array}{l}\text { How easy was the systematic } \\
\text { overview to use } \\
4 \text { point Likert scale } \\
1=\text { excellent } \\
4=\text { poor }\end{array}$ & $\begin{array}{l}\text { 1) } 33.7 \\
\text { 2) } 58.9 \\
\text { 3) } 4.2 \\
\text { 4) } 3.2 \\
\text { Mean }=1.77\end{array}$ & 0.68 & 95 & $\begin{array}{l}1.63- \\
1.91\end{array}$ \\
\hline Bandwagon effect & $\begin{array}{l}\text { 1) Perception of the } \\
\text { percentage of other health } \\
\text { unit using the systematic } \\
\text { overviews }\end{array}$ & 45.8 & 24.9 & 99 & $\begin{array}{l}40.87- \\
50.80\end{array}$ \\
\hline Bandwagon effect & $\begin{array}{l}\text { Percentage of other } \\
\text { colleagues in the same } \\
\text { position using the systematic } \\
\text { overviews }\end{array}$ & 46.9 & 26.6 & 105 & $\begin{array}{l}41.78- \\
52.10\end{array}$ \\
\hline
\end{tabular}




\begin{tabular}{|c|c|c|c|c|c|}
\hline $\begin{array}{l}\text { Individual } \\
\text { Characteristics }\end{array}$ & & & & & \\
\hline Current position & $\begin{array}{l}\text { Respondent's current } \\
\text { position }\end{array}$ & $\begin{array}{l}\text { Manager = } \\
54.6 \% \\
\text { Director = } \\
27.1 \% \\
\text { MOH = } \\
15.6 \% \\
\text { AMOH }=2.1 \% \\
\end{array}$ & & 140 & $\mathbf{N A}$ \\
\hline Tenure & years in current position & 7.3 & 4.9 & 141 & $\begin{array}{l}6.49- \\
8.12 \\
\end{array}$ \\
\hline Tenure & years in public health & 17.9 & 6.9 & 141 & $\begin{array}{l}16.83- \\
19.14\end{array}$ \\
\hline Education & 2) years since graduation & 13.8 & 8.4 & 141 & $\begin{array}{l}12.42- \\
15.24\end{array}$ \\
\hline Age & 10 year age categories & 50 years & 0.7 & 141 & NA \\
\hline Consultant contact & Access to research consultant & $\begin{array}{l}\text { Yes }=75.9 \% \\
\text { No }=24.1 \%\end{array}$ & & 141 & $\mathbf{N A}$ \\
\hline $\begin{array}{l}\text { Access to on-line } \\
\text { searching }\end{array}$ & $\begin{array}{l}\text { Direct access in organization } \\
\text { to on-line database searching }\end{array}$ & $\begin{array}{l}\text { Yes }=64.5 \% \\
\text { No }=33.3 \%\end{array}$ & & 141 & NA \\
\hline $\begin{array}{l}\text { Number of articles } \\
\text { retrieved in past } \\
\text { month }\end{array}$ & $\begin{array}{l}\text { 2) } \text { None } \\
2<10 \\
310-19 \\
420-29 \\
5>30\end{array}$ & $\begin{array}{l}7.1 \\
46.1 \\
31.2 \\
9.2 \\
6.4 \\
\text { Mean }=2.62 \\
\end{array}$ & 0.98 & 141 & $\begin{array}{l}2.45- \\
2.78\end{array}$ \\
\hline $\begin{array}{l}\text { Percentage of } \\
\text { retrieved articles } \\
\text { read on monthly } \\
\text { basis }\end{array}$ & $\begin{array}{l}1 \text { None } \\
2<25 \\
325-50 \\
451-75 \\
5>75 \%\end{array}$ & $\begin{array}{l}7.8 \\
17.0 \\
28.4 \\
21.3 \\
25.5 \\
\text { Mean }=3.40\end{array}$ & 1.25 & 141 & $\begin{array}{l}3.19- \\
3.61\end{array}$ \\
\hline Cosmopolitanism & $\begin{array}{l}\text { Days at external } \\
\text { meetings/conferences in past } \\
\text { year }\end{array}$ & 5.73 & 3.45 & 131 & $\begin{array}{l}5.13- \\
6.32\end{array}$ \\
\hline Authority & $\begin{array}{l}\text { Perceived barrier to using } \\
\text { research evidence based on } \\
\text { level of authority to make } \\
\text { program decisions } \\
5 \text { point Likert Scale } \\
1=\text { not a barrier } \\
5=\text { very serious barrier }\end{array}$ & $\begin{array}{l}\text { 1) } 37.9 \\
\text { 2) } 35.7 \\
\text { 3) } 18.6 \\
\text { 4) } 6.4 \\
\text { 5) } 1.4 \\
\text { Mean = } 1.98\end{array}$ & 0.98 & 140 & $\begin{array}{l}1.82- \\
2.14\end{array}$ \\
\hline
\end{tabular}




\begin{tabular}{|c|c|c|c|c|c|}
\hline Critical appraisal & $\begin{array}{l}\text { Perceived barrier to using } \\
\text { research evidence based on } \\
\text { critical appraisal skills } \\
5 \text { point Likert Scale } \\
1=\text { not a barrier } \\
5=\text { very serious barrier }\end{array}$ & $\begin{array}{l}\text { 1) } 19.1 \\
\text { 2) } 30.5 \\
\text { 3) } 34.8 \\
\text { 4) } 14.2 \\
\text { 5) } 1.4 \\
\text { Mean }=2.48\end{array}$ & 1.0 & 141 & $\begin{array}{l}2.32- \\
2.65\end{array}$ \\
\hline Financial Resources & $\begin{array}{l}\text { Perceived barrier to using } \\
\text { research evidence based on } \\
\text { limited financial resources } \\
5 \text { point Likert Scale } \\
\text { I= not a barrier } \\
5=\text { very serious barrier }\end{array}$ & $\begin{array}{l}\text { 1) } 7.1 \\
\text { 2) } 12.9 \\
\text { 3) } 32.1 \\
\text { 4) } 37.9 \\
\text { 5) } 10.0 \\
\text { Mean = 3.31 }\end{array}$ & 1.05 & 140 & $\begin{array}{l}3.13- \\
3.48\end{array}$ \\
\hline $\begin{array}{l}\text { Prior use of } \\
\text { systematic } \\
\text { overviews } \\
\end{array}$ & $\begin{array}{l}\text { Have used systematic } \\
\text { overviews in the past to } \\
\text { make program decisions }\end{array}$ & $\begin{array}{l}\text { Yes }=39.7 \% \\
\text { No }=60.2 \%\end{array}$ & & 77 & NA \\
\hline Future Use & $\begin{array}{l}\text { Expectation of using the } \\
\text { systematic overview in } \\
\text { future }\end{array}$ & $\begin{array}{l}\text { Yes }=84.7 \% \\
\text { No }=15.2 \%\end{array}$ & & 118 & NA \\
\hline
\end{tabular}

(Numbers 1-5 in table correspond to response options on Likert scale in questionnaires) 


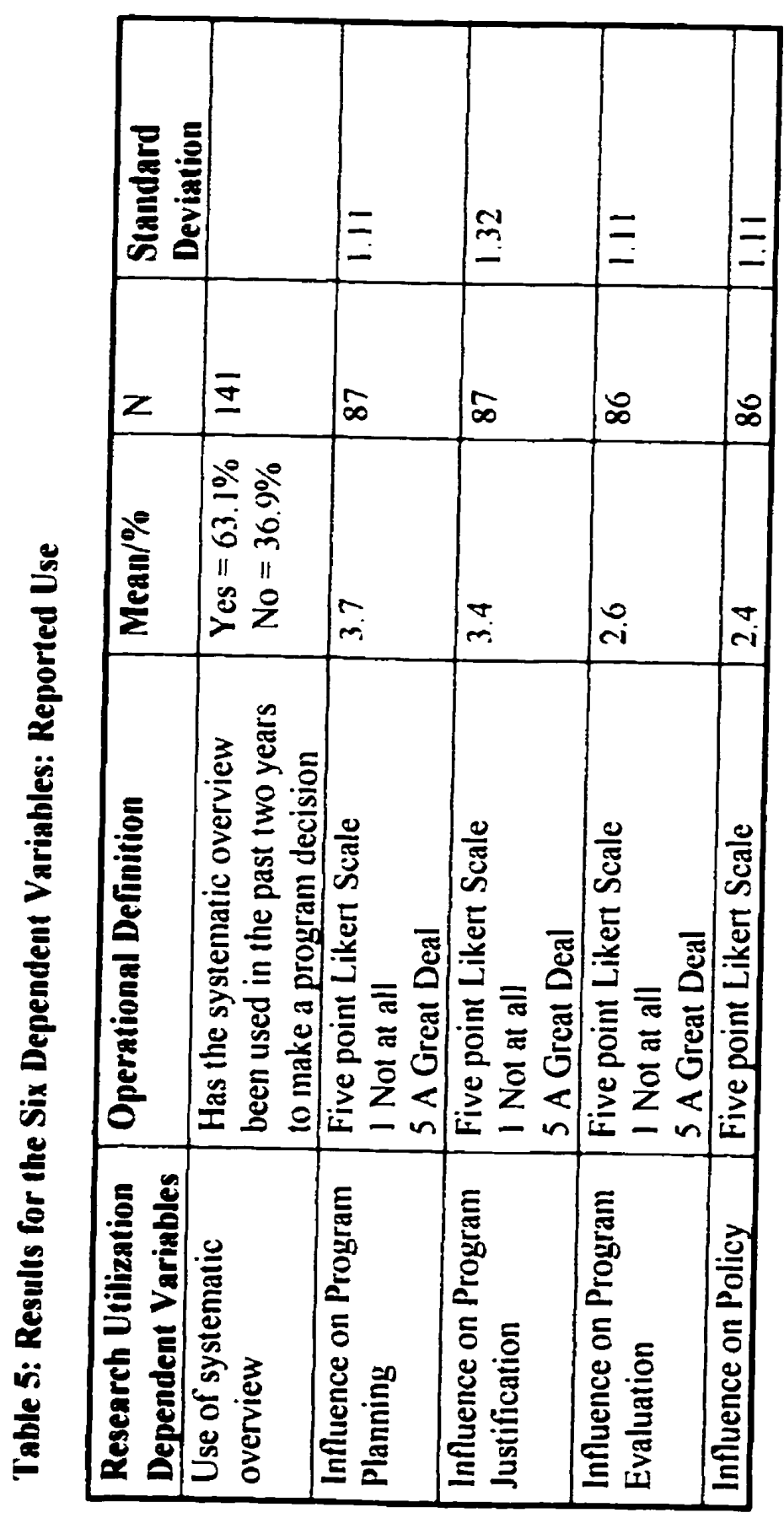




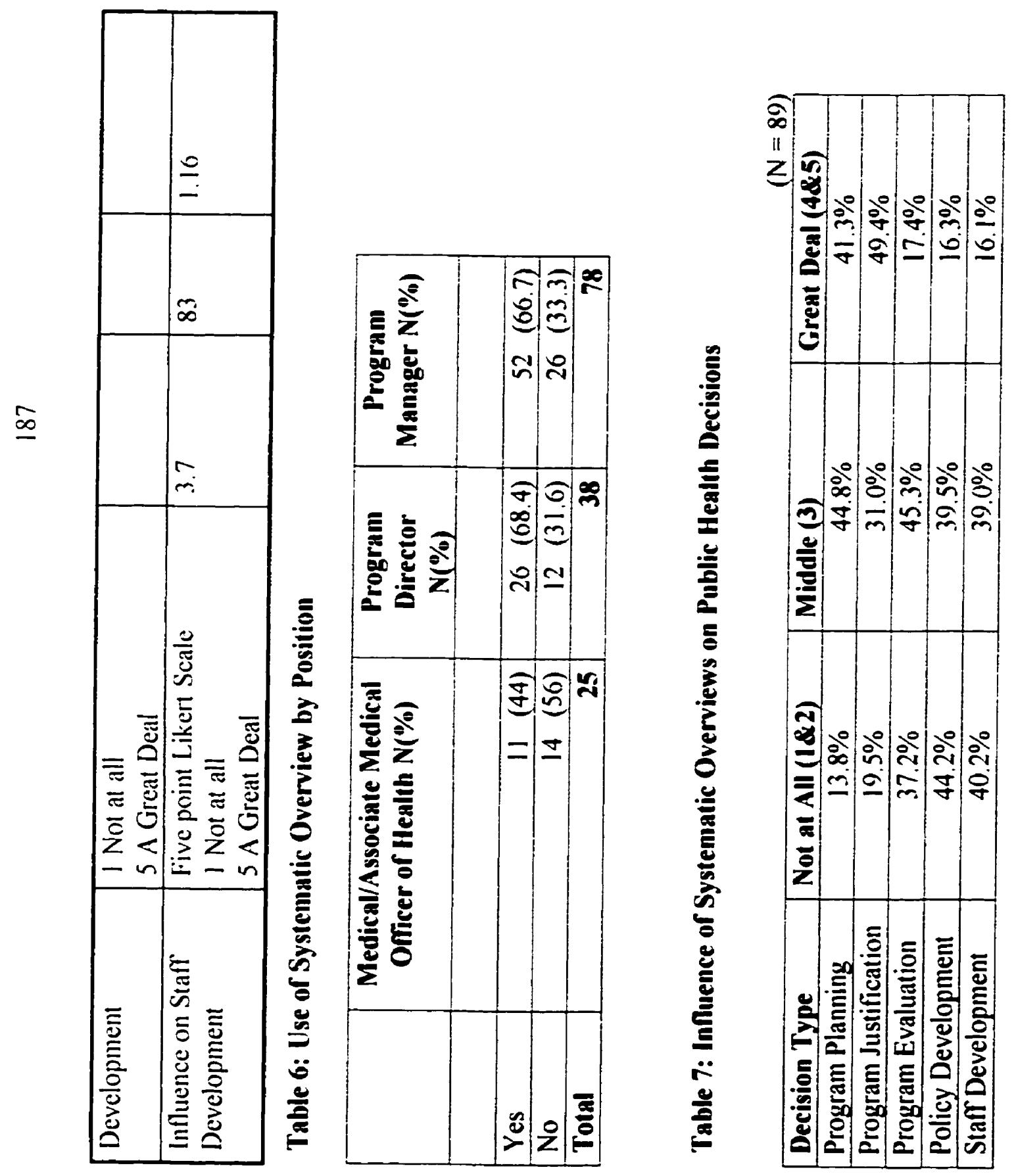


Table 8: Dependent and Independent Correlation Analysis

\begin{tabular}{|c|c|c|c|c|c|c|}
\hline & $\begin{array}{l}\text { Overall } \\
\text { Use }\end{array}$ & $\begin{array}{l}\text { Progianin } \\
\text { Panning }\end{array}$ & $\begin{array}{l}\text { Program } \\
\text { Justification }\end{array}$ & $\begin{array}{l}\text { Program } \\
\text { Evaluation }\end{array}$ & Policy Developmencent & Stuff Developme \\
\hline Policy udherence & (1)18 & .033 & .176 & .123 & .097 & $1+1$ \\
\hline Organizational valuc & .099 & $.332 * *$ &.$(1) 5^{* *}$ & $.232^{*}$ & $3+9 * *$ & $2.30 *$ \\
\hline Alkequate resources & .12 & $.249^{*}$ & $.221^{*}$ & .164 & $.334 *$ & .034 \\
\hline Staft training & $.1+3$ & $.31 .3^{* *}$ &. $.350 * *$ & $245^{*}$ & $.3+10 * *$ & .119 \\
\hline Integrative mechanisms & .158 & .1110 & .139 & .22()$^{*}$ & $.251 *$ & .097 \\
\hline Extent info searched for & .112 & .123 & $-.289 * *$ & .149 & 205 & .078 \\
\hline $\begin{array}{l}\text { Importance of research evidence } \\
\text { in HU }\end{array}$ & .086 & .109 & -.195 & .134 & .192 & .049 \\
\hline Regulations and legislation & 122 & .023 & .028 & $.11+4$ & .111 & .011 \\
\hline Collaboration & .092 & .212 & $.217^{*}$ & .08 .3 & $26)^{*}$ & $.36 .3^{* *}$ \\
\hline MOH influence over board & .024 & .211 & .186 & .1124 & .194 & $.267^{*}$ \\
\hline MOH influence over politicians & .005 & .033 & .117 & .124 & .019 & .135 \\
\hline Board infuenced by rewearch & -.093 & .021 & .160 & .151 & .176 & .120 \\
\hline $\begin{array}{l}\text { Politicians infuenced hy } \\
\text { reseurch }\end{array}$ & .066 & .085 & $.266^{*}$ & .029 & $.274^{*}$ & .157 \\
\hline \# FTE's & .0112 & .22 .3 & .105 & .093 & .075 & 204 \\
\hline Population served & 0.0 .32 & .045 & .056 & $.234^{*}$ & $.0(1)$ & .038 \\
\hline Number of divisions & .06 .5 & .081 & .084 & .0 .57 & .104 & .184 \\
\hline Number of programs & .016 & .006 & .143 & .134 & .017 & .106 \\
\hline Levels of workers & 006 & .153 & .053 & .210 &.$(194$ & $.313^{* *}$ \\
\hline Number of research projects & .008 & .171 & .069 & .0157 & .061 & .177 \\
\hline Involved in rescarch projects & .003 .5 & .061 & .1120 & .069 & .030 & .129 \\
\hline Teuching heulth unit stutus & .110 & .162 & .062 & .026 & .0 .34 & .190 \\
\hline Position at baveline & $.212^{*}$ & .080 & .137 & .037 & .075 & .042 \\
\hline Years since graduation & .052 & .204 & $1+4$ & .087 & 115 & 124 \\
\hline Agc & .022 & $.251)^{*}$ & 110 & .115 & $297 * *$ & .168 \\
\hline Yeurs in current position & .077 & .105 & .155 & .044 & .156 & .112 \\
\hline Years in public heulth & $.0+4$ & .180 & .079 & .2112 & .159 & .2117 \\
\hline Access to consultant & .18811 & .1190 & .031 & .011 & .132 & .076 \\
\hline Number articles netrieved & .169 & .064 & .129 & .180 & $.051)$ & .018 \\
\hline Number articles read & .029 & $.255^{*}$ & $28(1 * *$ & .145 & .164 & 124 \\
\hline Number days at conferences & .152 & .122 & .011 & $.1+5$ & .078 & $.316 * *$ \\
\hline $\begin{array}{l}\text { Authority a barrier to using } \\
\text { reweanch }\end{array}$ &.$(0) 3$ & .0108 & .046 & .025 & .045 & .016 \\
\hline
\end{tabular}




\begin{tabular}{|c|c|c|c|c|c|c|}
\hline & $\begin{array}{l}\text { Overall } \\
\text { Ise. }\end{array}$ & $\begin{array}{l}\text { Program } \\
\text { Planning }\end{array}$ & $\begin{array}{l}\text { Program } \\
\text { Justification }\end{array}$ & $\begin{array}{l}\text { Progratum } \\
\text { Eraluation }\end{array}$ & Policy Development & Stuff Developme \\
\hline $\begin{array}{l}\text { Critical appraisal harrier to } \\
\text { using research }\end{array}$ & .093 & .010 & .012 & .053 & .063 & .055 \\
\hline $\begin{array}{l}\text { Rewources a barrier to using } \\
\text { research }\end{array}$ & .066 & .074 & .05 .3 & .054 & $(011$ & .116 \\
\hline $\begin{array}{l}\text { Access to on-line database } \\
\text { searching }\end{array}$ & .115 & -.118 & .110 .5 & .115 & $-.278^{* *}$ & .0132 \\
\hline $\begin{array}{l}\text { Priority should be given } 10 \\
\text { overviews T1 }\end{array}$ & .091 & $.06(1)$ & .038 & .0104 & .010 & .131 \\
\hline Weight given to reucurch & .115 & .0108 & .021 & .0105 & .060 & .024 \\
\hline $\begin{array}{l}\text { Weight should be given to } \\
\text { reveunch }\end{array}$ & .061 & $.1+10$ & .052 & .153 & .051 & 125 \\
\hline $\begin{array}{l}\text { Overviews overcome access } \\
\text { burrier T1 }\end{array}$ & .031 & .140 & .078 & .008 & .097 & .126 \\
\hline Priority given to overviews T2 & .031 & .183 & .013 & .068 & .212 & .007 \\
\hline $\begin{array}{l}\text { Overviews overcome time } \\
\text { harrier TI }\end{array}$ & .039 & .109 & .021 & .045 & $.1+1$ & .100 \\
\hline $\begin{array}{l}\text { Overviews overcome critical } \\
\text { appraisal harrice } \mathrm{T1}\end{array}$ & .132 & $.212^{*}$ & .1198 & .112 & .087 & .058 \\
\hline $\begin{array}{l}\text { Overviews overcome rewource } \\
\text { harrier T1 }\end{array}$ & .086 & .010 & .1054 & .204 & $.23 .5^{*}$ & .180 \\
\hline $\begin{array}{l}\text { Overviews overeome relevance } \\
\text { barrier T1 }\end{array}$ & .084 & .0014 & .0 .54 & $.254^{*}$ & .017 & $.07(0)$ \\
\hline $\begin{array}{l}\text { Overviews overcome } \\
\text { unsupportive work environment } \\
\text { barrier T1 }\end{array}$ & .081 & $.220 *$ & .037 & .160 & .024 & .128 \\
\hline $\begin{array}{l}\text { Overviews overcome authority } \\
\text { barrier TI }\end{array}$ & $.0+5$ & .148 & .119 & $288^{* *}$ & .062 & $.317^{* *}$ \\
\hline $\begin{array}{l}\text { Overviews overcome authority } \\
\text { barrier T2 }\end{array}$ & .025 & .064 & .048 & .098 & .135 & .186 \\
\hline $\begin{array}{l}\text { Overview's overcome critical } \\
\text { appraisal harrier 'T2 }\end{array}$ & $.254 * *$ & $.258^{*}$ & .189 & .071 & .032 & .0111 \\
\hline $\begin{array}{l}\text { Overviews overcome limited } \\
\text { access barrier } \mathrm{T} 2\end{array}$ & $.192^{*}$ & .064 & .146 & .005 & .078 & .052 \\
\hline $\begin{array}{l}\text { Overviews overcome time } \\
\text { harrier T2 }\end{array}$ & $.284 * *$ & $.261^{*}$ & $285^{*}$ & .082 & .131 & .170 \\
\hline $\begin{array}{l}\text { Overviews overcome rewource } \\
\text { harrier T2 }\end{array}$ & .038 & .090 & .0 .57 & $.234 *$ & $2.56^{*}$ & .158 \\
\hline
\end{tabular}



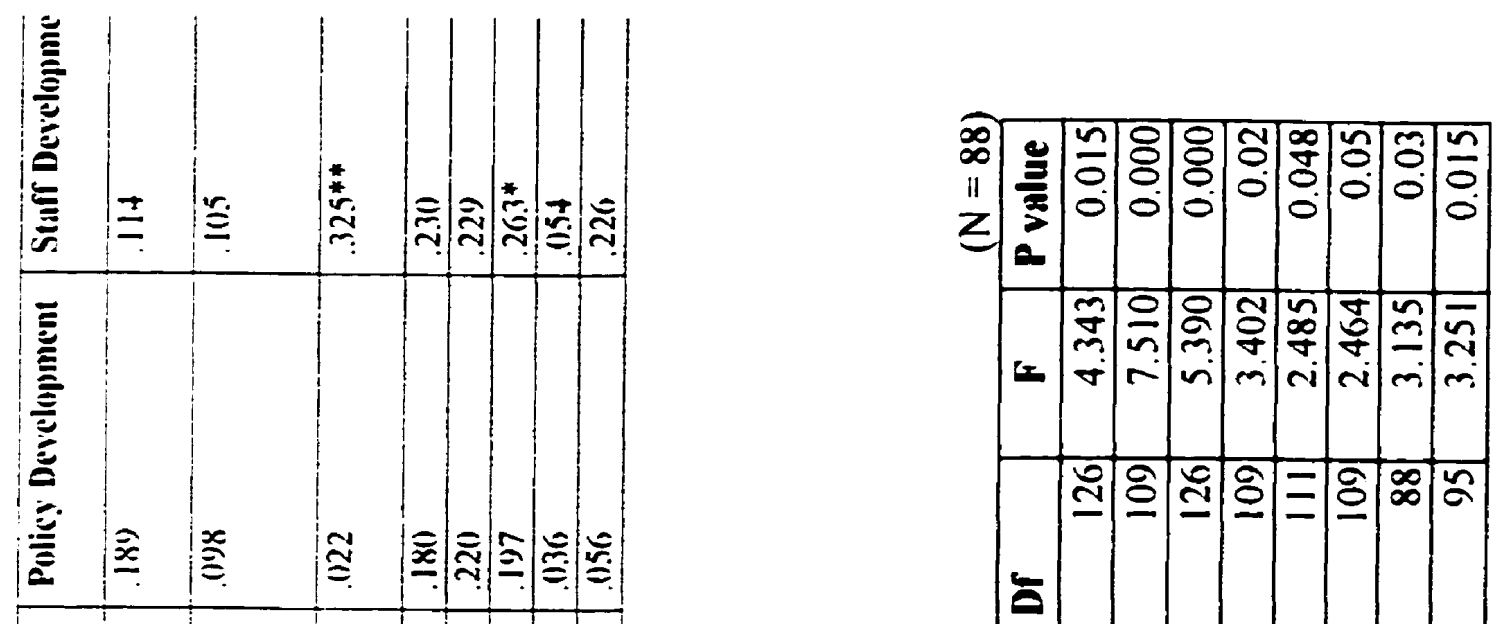

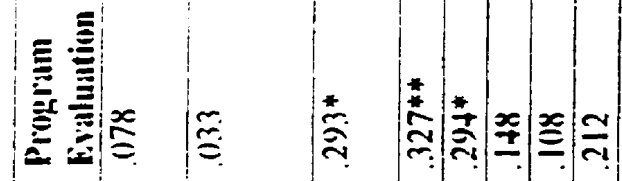

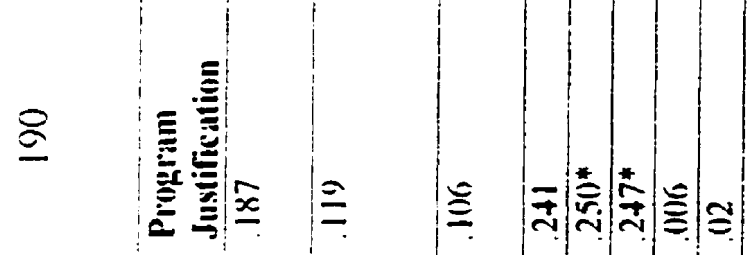
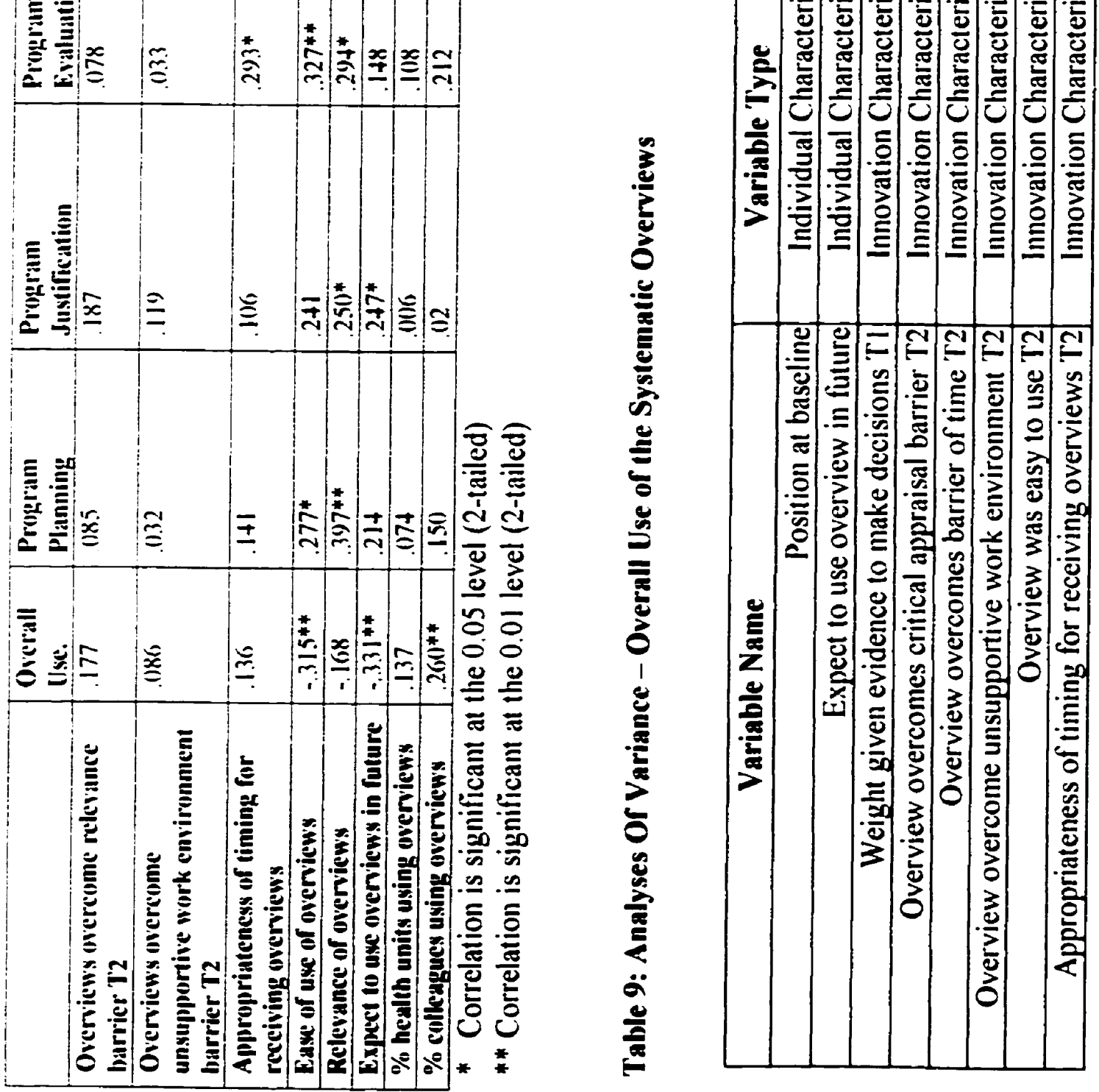

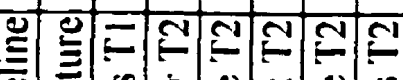
言

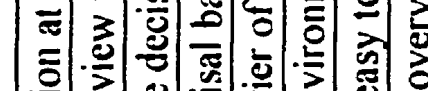
:을

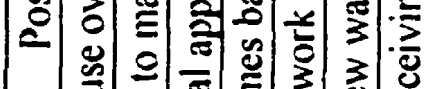

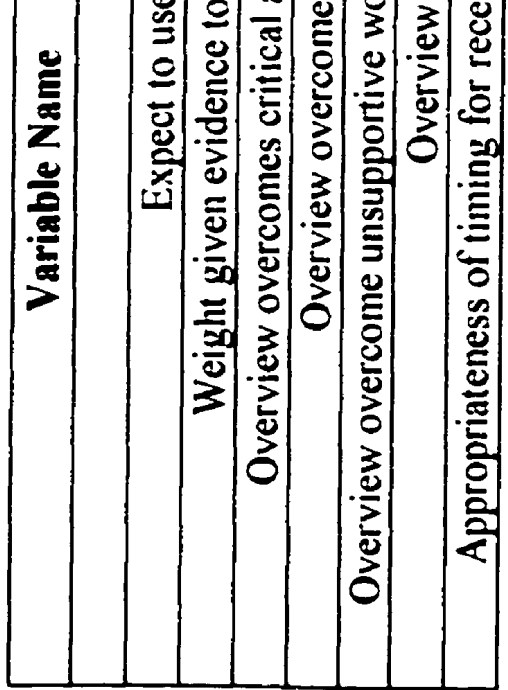




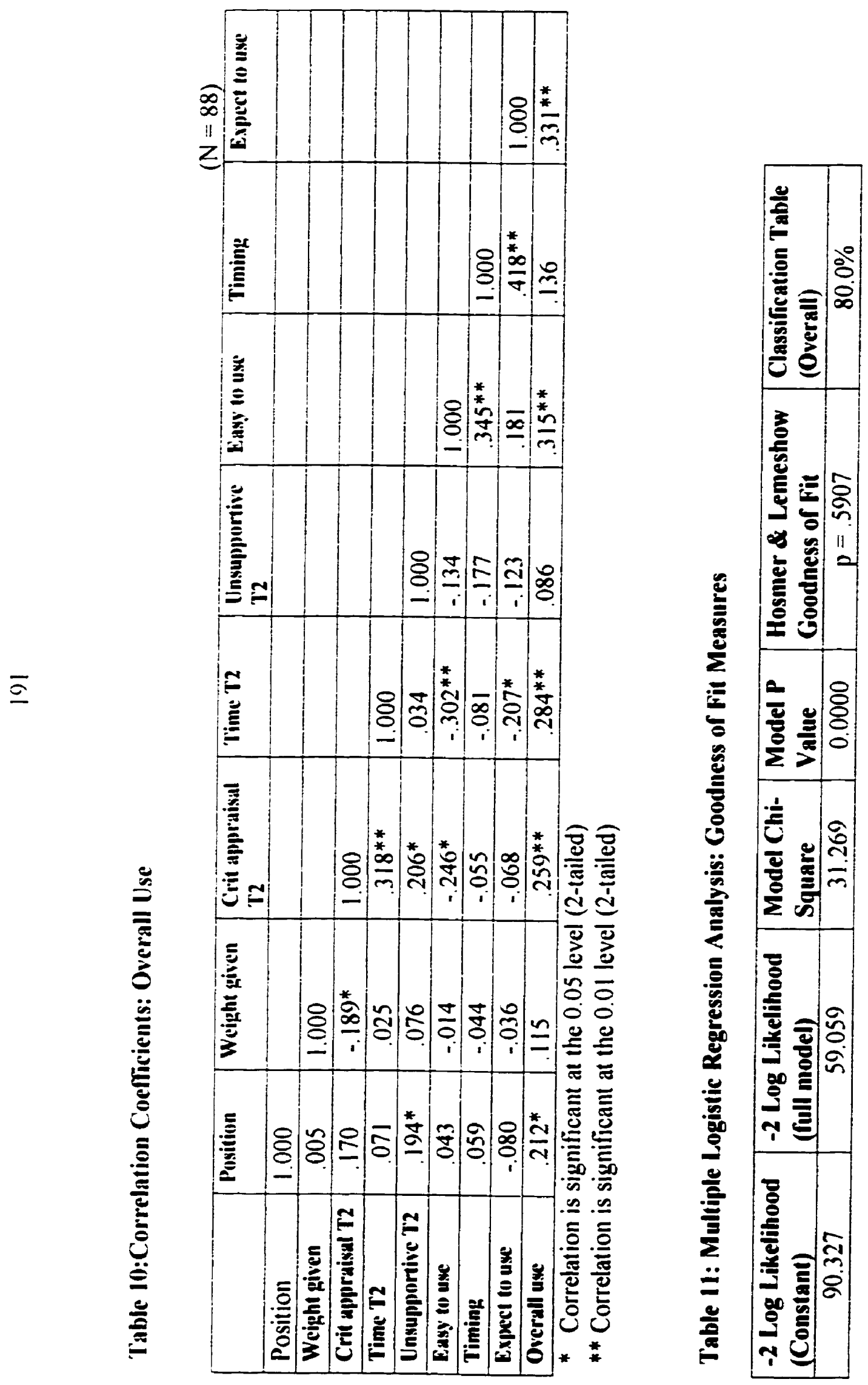


Table 12: Final Iogistic Regression Analysis - Overall Use

\begin{tabular}{|c|c|c|c|c|c|c|c|c|c|}
\hline & & & & & & & & \multicolumn{2}{|c|}{$(N=88)$} \\
\hline Variable & B & S.E. & Wald & Dr & Sig & $\mathbf{R}$ & $\begin{array}{c}\text { Ouds } \\
\text { Ratio } \\
\text { Exp (B) }\end{array}$ & $\begin{array}{l}\text { Lower } \\
95 \% \text { CI } \\
\text { Exp (B) }\end{array}$ & $\begin{array}{l}\text { Upper } \\
95 \% \text { CI } \\
\operatorname{Exp}(\mathrm{B})\end{array}$ \\
\hline Expect to use & 1.66369 & .6140 & 7.3404 & 1 & .0067 & .2432 & 1895 & .0569 & .6312 \\
\hline Easy to use & 1.1038 & .5813 & 3.6053 & 1 & .0567 & .1333 & .3316 & .1061 & 1.0362 \\
\hline $\begin{array}{l}\text { Critical } \\
\text { appraisal }\end{array}$ & 1.2502 & .4624 & 7.3099 & 1 & .0069 & .2425 & 3.4910 & 1.4104 & 8.6407 \\
\hline Moh & & & 8.1878 & 2 & .0167 & .2153 & & & \\
\hline Directors & 2.5376 & 1.0140 & 6.2634 & 1 & 0.0123 & 2173 & 12.6496 & 1.7338 & 92.2918 \\
\hline Managers & 2.4257 & .9030 & 7.2154 & 1 & 0.0072 & 2403 & 11.3099 & 1.9266 & 66.3934 \\
\hline Constant & .7000 & 2.1888 & .1023 & 1 & .7491 & & & & \\
\hline
\end{tabular}

Table 13: Influence of Systematic Overviews on Program Planning: Analysis of Variance

\begin{tabular}{|l|l|r|r|r|}
\hline \multicolumn{1}{|c|}{ Independent Variable } & \multicolumn{1}{|c|}{ Variable Type } & Df & \multicolumn{1}{c|}{ F } & P value \\
\hline Value organization placed on evidence & Organizational & 86 & 5.564 & 0.002 \\
\hline Ongoing training & Organizational & 86 & 2.607 & 0.042 \\
\hline \# FTE's & Organizational & 68 & 2.373 & 0.006 \\
\hline Age category & Individual & 86 & 3.634 & 0.031 \\
\hline \# articles retrieved & Individual & 86 & 3.283 & 0.015 \\
\hline \# articles read & Individual & 86 & 2.623 & 0.041 \\
\hline Overview can overcome critical appraisal T2 & Innovation & 77 & 4.525 & 0.006 \\
\hline Overview can overcome time T2 & Innovation & 77 & 4.685 & 0.012 \\
\hline Overview relevance T2 & Innovation & 66 & 6.095 & 0.001 \\
\hline
\end{tabular}




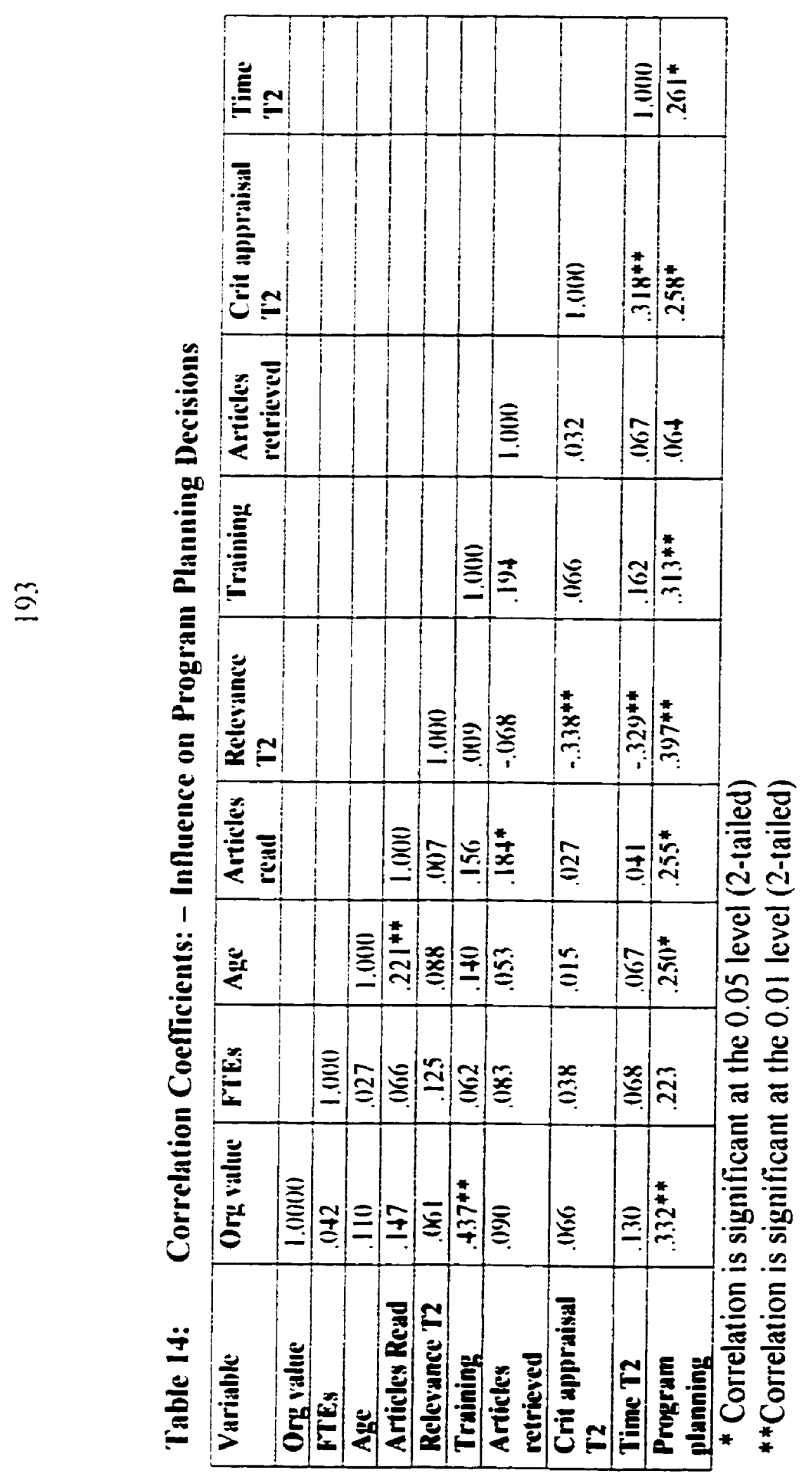




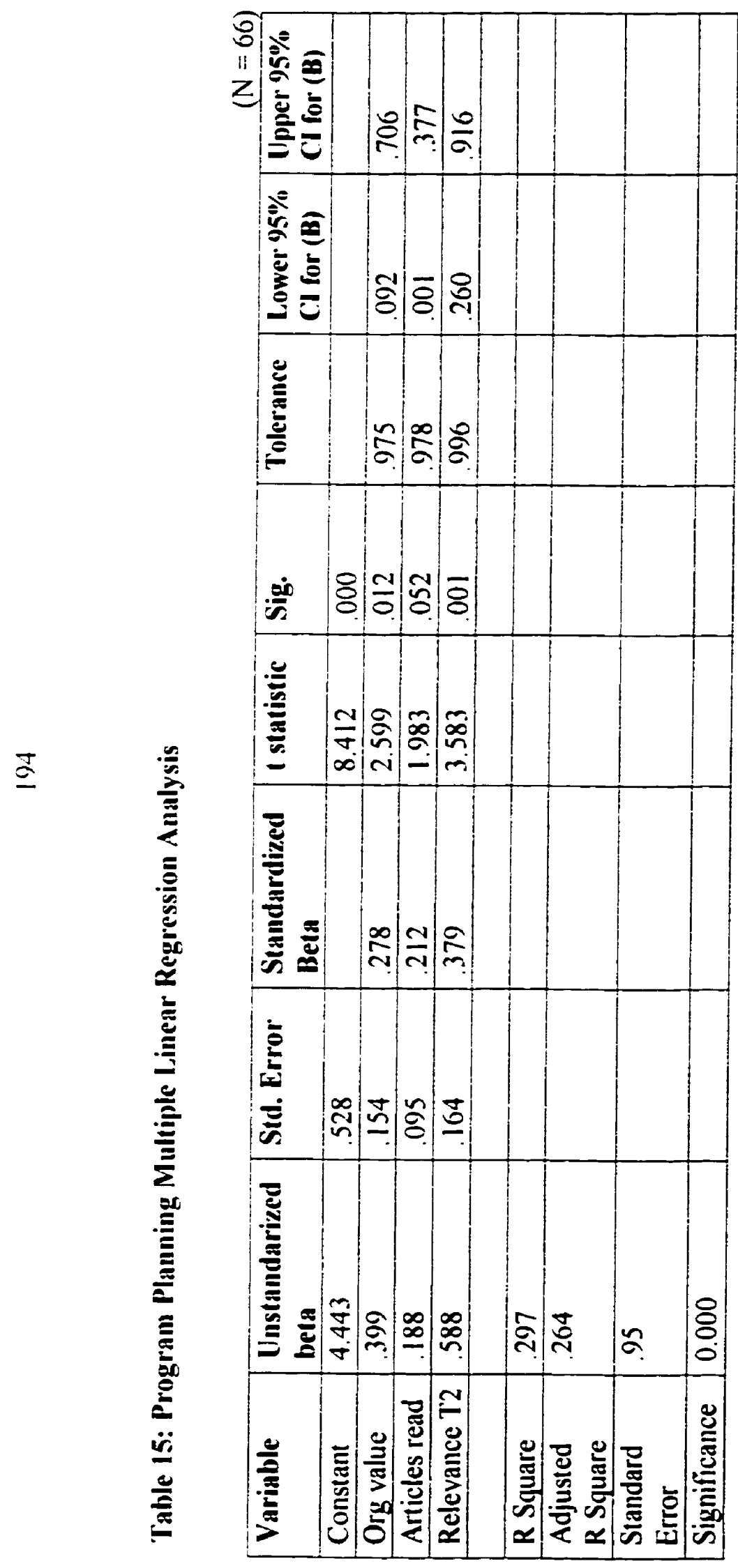


Table 16: Influence of Systematic Overviews on Program Justification: Results of Annlysis of Variance:

\begin{tabular}{|l|l|r|r|r|}
\hline \multicolumn{1}{|c|}{ Independent Variable } & Variable Type & \multicolumn{1}{c|}{ Df } & F & p value \\
\hline Perceived value organization placed on research & Organizational & 86 & 7.258 & 0.000 \\
\hline Perceived adequate financial resources & Organizational & 86 & 2.465 & 0.051 \\
\hline Perceived ongoing training in research methods & Organizational & 86 & 4.203 & 0.004 \\
\hline \# Full time Equivalent Staff & Organizational & 68 & 1.702 & 0.052 \\
\hline Extent information is looked for before making decisions & Organizational & 86 & 2.960 & 0.024 \\
\hline Perceived collaboration with other local agencies & Environmental & 86 & 3.844 & 0.013 \\
\hline Perceived MOH influence over local board of health & Environmental & 84 & 2.573 & 0.044 \\
\hline Perception politicians influenced by research & Environmental & 81 & 3.457 & 0.012 \\
\hline \% of retrieved articles read in past month & Individual & 86 & 2.877 & 0.028 \\
\hline Expect to use overviews in future & Individual & 75 & 4.754 & 0.011 \\
\hline Perception overviews overcome time barrier T2 & Innovation & 77 & 3.672 & 0.03 \\
\hline
\end{tabular}


Table 17: Bivariate Correlation Coefficients - Influcnce on Program Justification

\begin{tabular}{|c|c|c|c|c|c|c|c|c|c|c|c|}
\hline & $\begin{array}{l}\text { Org } \\
\text { value }\end{array}$ & $\begin{array}{l}\text { Adevuatc } \\
\text { remourcess }\end{array}$ & Training & Fres & $\begin{array}{l}\text { Seurch for } \\
\text { information }\end{array}$ & Collaboration & $\begin{array}{l}\text { MOHI } \\
\text { influences } \\
\text { board }\end{array}$ & $\begin{array}{l}\text { Politicians } \\
\text { influcenced }\end{array}$ & $\begin{array}{l}\text { Articles } \\
\text { read }\end{array}$ & $\begin{array}{l}\text { Expect } \\
\text { to use }\end{array}$ & Time \\
\hline Org value & 1.000 & & & & & & & & & & \\
\hline Ady remurces & $431^{* *}$ & 1.000 & & & & & & & & & \\
\hline Training & $.437^{* *}$ & $435^{* *}$ & 1.000 & & & & & & & & \\
\hline FTEs & .042 & .104 & .062 & 1.000 & & & & & & & \\
\hline $\begin{array}{l}\text { Seurch for } \\
\text { info }\end{array}$ & $.214^{*}$ & $.176^{*}$ & .149 & .089 & 1.000 & & & & & & \\
\hline Collaborution & $295^{* *}$ & $.185^{*}$ & $202^{*}$ & .021 & .049 & 1.000 & & & & & \\
\hline $\begin{array}{l}\text { MOH } \\
\text { influences } \\
\text { moard } \\
\end{array}$ & $.283^{* *}$ & .147 & $257^{* *}$ & $.201 *$ & .122 & $.265^{* *}$ & 1.000 & & & & \\
\hline $\begin{array}{l}\text { Politicians } \\
\text { influenced }\end{array}$ & $.316^{* *}$ & $.290^{* *}$ & $.282 * *$ & .137 & $.246^{* *}$ & $.328 * *$ & $247^{* *}$ & 1.000 & & & \\
\hline Articles read & .147 & .082 & .156 & .066 & $.10 \overline{5}$ & .011 & .034 & .056 & 1.000 & & \\
\hline Expect to usc & .036 & .086 & .005 & .120 & .105 & .10 & .047 & .108 & .080 & 1.000 & \\
\hline Time T2 & .130 & .02 & 162 & .068 & .014 & .021 & .105 & 140 & .041 & $.207^{*}$ & 1.000 \\
\hline $\begin{array}{l}\text { Program } \\
\text { Justification }\end{array}$ & $.405^{* *}$ & $.221^{*}$ & $.359 * *$ & .105 & $.289^{*}$ & $.217^{*}$ & .086 & $.266^{*}$ & $.280^{* *}$ & $.247^{*}$ & $.285^{*}$ \\
\hline
\end{tabular}

* Correlation is significant at the 0.05 level (2-tailed)

**Correlation is significant at the 0.01 level (2-tailed) 


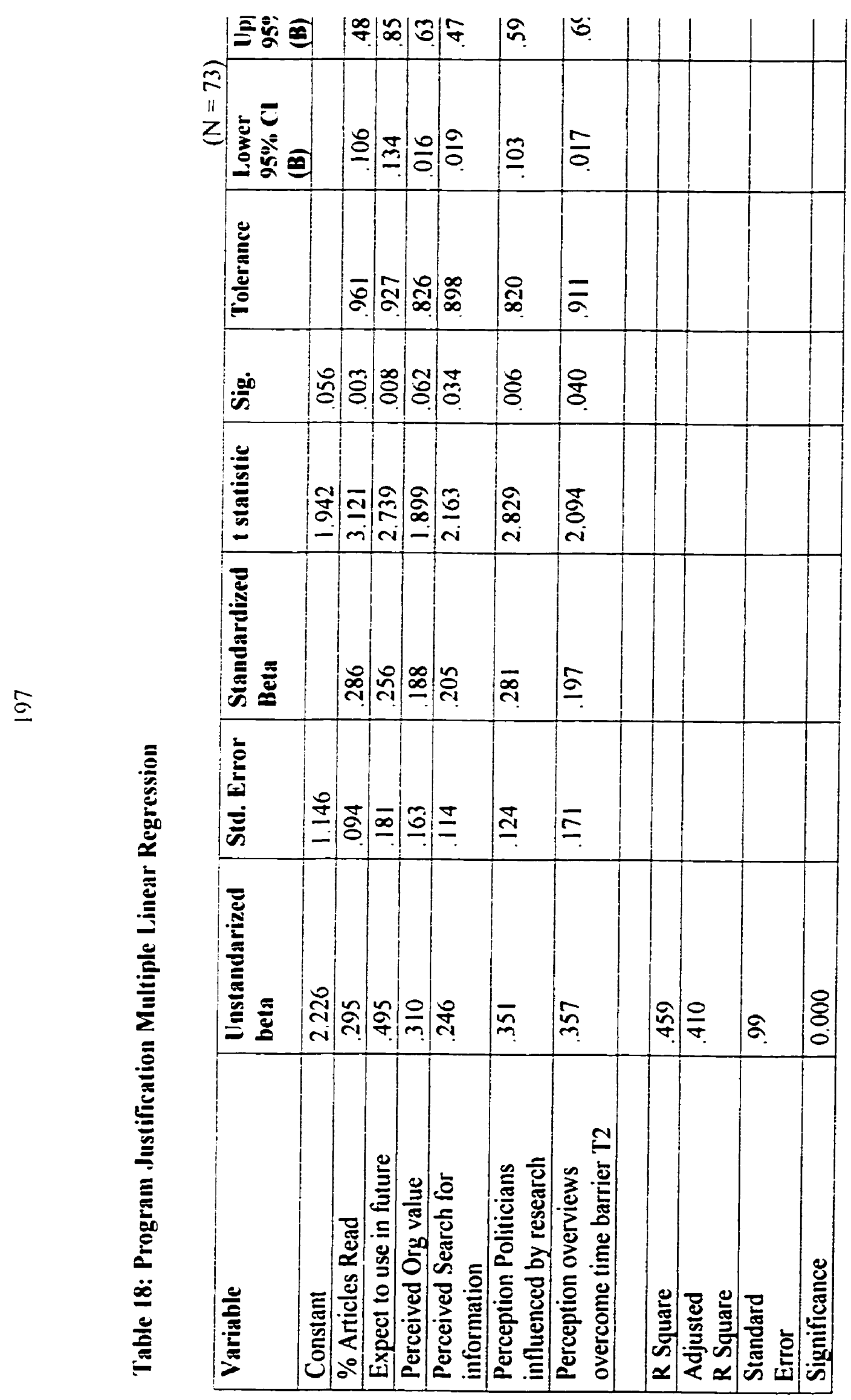


Table 19: Infuence of Systematic Overviews on Program Evaluation: Results of Analysis of Variance

\begin{tabular}{|l|l|r|r|r|}
\hline \multicolumn{1}{|c|}{ Independent Variable } & \multicolumn{1}{|c|}{ Variable Type } & \multicolumn{1}{c|}{ Df } & \multicolumn{1}{c|}{ F } & \multicolumn{1}{c|}{ P value } \\
\hline \# of programs offered by health unit & Organizational & 85 & 1.949 & 0.046 \\
\hline$\%$ of articles read in past month & Individual & 85 & 4.515 & 0.002 \\
\hline $\begin{array}{l}\text { \# days at conference in past year } \\
\text { Perceived relevance at T2 to decisions } \\
\text { being made }\end{array}$ & Individual & 79 & 1.920 & 0.052 \\
\hline
\end{tabular}

Table 20: Bivariate Correlation Coefficients- lufluence on Program Evaluation

\begin{tabular}{|l|l|l|l|l|}
\hline & \# of programs & Articles read & Days at conferences & Relevance T2 \\
\hline \# of progranis & 1.000 & & & \\
\hline Articles read & $171^{*}$ & 1.000 & & \\
\hline Days at conferences & .149 & .080 & 1.000 & 1.000 \\
\hline Relevance T2 & .060 & .007 & .051 & $.294^{*}$ \\
\hline Program Evaluation & .134 & .145 & 145 & \\
\hline
\end{tabular}

* Correlation is significant at the 0.05 level (2-tailed) 
Table 21: Program Evaluation Multiple Linear Regression

\begin{tabular}{|c|c|c|c|c|c|c|c|}
\hline \multirow[b]{2}{*}{ Variable } & \multirow[b]{2}{*}{ Unstandarized beta } & \multirow[b]{2}{*}{ Std. Eirror } & \multirow[b]{2}{*}{$\begin{array}{l}\text { Standardized } \\
\text { Beta }\end{array}$} & \multirow[b]{2}{*}{ t statistic } & \multirow[b]{2}{*}{ Sig. } & \multicolumn{2}{|c|}{$(N=64)$} \\
\hline & & & & & & $\begin{array}{l}\text { Lower } \\
95 \% \mathrm{CI} \\
\text { (B) }\end{array}$ & $\begin{array}{l}\text { Upper } \\
95 \% \text { CI } \\
\text { (B) }\end{array}$ \\
\hline Constant & 3.517 & .362 & & 9.720 & 0.000 & & \\
\hline $\begin{array}{l}\text { Perceived Relevance of } \\
\text { overviews T2 }\end{array}$ & .556 & .194 & .339 & 2.861 & 0.006 & .166 & .944 \\
\hline & & & & & & & \\
\hline R Square & .115 & & & & & & \\
\hline $\begin{array}{l}\text { Adjusted } \\
\text { R Square }\end{array}$ & .101 & & & & & & \\
\hline $\begin{array}{l}\text { Standard } \\
\text { Error }\end{array}$ & 1.06 & & & & & & \\
\hline Significance & 0.006 & & & & & & \\
\hline
\end{tabular}

Table 22: Influence of Systematic Overviews on Policy Development: Results of Analysis of Variance:

\begin{tabular}{|l|l|r|r|r|}
\hline \multicolumn{1}{|c|}{ Independent Variable } & \multicolumn{1}{|c|}{ Variable Type } & \multicolumn{1}{c|}{ df } & \multicolumn{1}{c|}{ F } & p value \\
\hline Perceived value organization placed on research & Organizational & $\mathbf{8 5}$ & 4.399 & 0.006 \\
\hline Perceived adequate financial resources & Organizational & 85 & 3.290 & .015 \\
\hline Perceived ongoing training in research methods & Organizational & 85 & 2.864 & 0.028 \\
\hline Perceived importance of research in program decisions & Organizational & 85 & 2.827 & 0.03 \\
\hline Perception local politicians influenced by research & Environmental & 80 & 6.352 & .000 \\
\hline Age category & Individual & 85 & 4.016 & 0.022 \\
\hline Direct access to on-line searching & Individual & $\mathbf{8 5}$ & 7.015 & 0.010 \\
\hline
\end{tabular}


Table 23: Bivariate Correlation Coefficients - Influence on Policy Development

\begin{tabular}{|c|c|c|c|c|c|c|c|}
\hline & Org value & $\begin{array}{l}\text { Adq } \\
\text { resources }\end{array}$ & Training & $\begin{array}{l}\text { Importance of } \\
\text { research }\end{array}$ & $\begin{array}{l}\text { Politicians } \\
\text { influenced }\end{array}$ & Age & $\begin{array}{l}\text { On line } \\
\text { database }\end{array}$ \\
\hline Org value & 1.000 & & & & & & \\
\hline Adq resources & $431^{* *}$ & 1.000 & & & & & \\
\hline Training & $437^{* *}$ & $435 * *$ & 1.000 & & & & \\
\hline $\begin{array}{l}\text { Importance of } \\
\text { research }\end{array}$ & $340^{* *}$ & $.355^{* *}$ & $.237^{* *}$ & 1.000 & & & \\
\hline Politicians influenced & $.316^{* *}$ & $.290^{* *}$ & $282^{* *}$ & $.244^{* *}$ & 1.000 & & \\
\hline Age & 110 & $179^{*}$ & .140 & .156 & .150 & 1.000 & \\
\hline On line database & .149 & .152 & $.219^{* *}$ & $304^{* *}$ & $.171^{*}$ & .108 & 1.000 \\
\hline Policy Development & $349^{* *}$ & $.334^{* *}$ & $.349^{* *}$ & .192 & $.274^{*}$ & $297^{* *}$ & $-.278^{* *}$ \\
\hline
\end{tabular}

* Correlation is significant at the 0.01 level (2-tailed)

** Correlation is significant at the 0.05 level (2-tailed) 
Table 24: Policy Development Multiple Linear Regression Analysis

\begin{tabular}{|c|c|c|c|c|c|c|c|c|}
\hline Variable & $\begin{array}{l}\text { Unstandarized } \\
\text { beta }\end{array}$ & $\begin{array}{l}\text { Standard } \\
\text { Errror }\end{array}$ & $\begin{array}{l}\text { Standardized } \\
\text { Beta }\end{array}$ & I statistic & Sig. & Tolerance & $\begin{array}{l}\text { Lower } 95 \% \\
\text { CI (B) }\end{array}$ & $\begin{array}{l}\text { Upper } \\
\text { CI (B) }\end{array}$ \\
\hline Constant & 1.446 & .511 & & 2.831 & 0.006 & & & \\
\hline Age category & .475 & .139 & .318 & 3.422 & 0.001 & .984 & .199 & .752 \\
\hline On-line access & .516 & .203 & .237 & 2.538 & 0.013 & .973 & .111 & .921 \\
\hline $\begin{array}{l}\text { Perceived Org } \\
\text { value }\end{array}$ & .467 & .128 & .340 & 3.636 & 0.000 & .971 & .211 & .722 \\
\hline & & & & & & & & \\
\hline R Square & .330 & & & & & & & \\
\hline $\begin{array}{l}\text { Adjusted } \\
\text { R Square } \\
\end{array}$ & .305 & & & & & & & \\
\hline Standard Error & .89 & & & & & & & \\
\hline Significance & 0.000 & & & & & & & \\
\hline
\end{tabular}

Table 25: Infuence of Systenatic Overviews on Staff Development: Results of Analysis of Variance:

\begin{tabular}{|l|l|r|r|r|}
\hline \multicolumn{1}{|c|}{ Independent Variable } & \multicolumn{1}{|c|}{ Variable Type } & df & \multicolumn{1}{|c|}{ F } & p value \\
\hline $\begin{array}{l}\text { Perceived appropriateness of the timing for receiving } \\
\text { overviews }\end{array}$ & Innovation & 65 & 2.648 & 0.042 \\
\hline
\end{tabular}




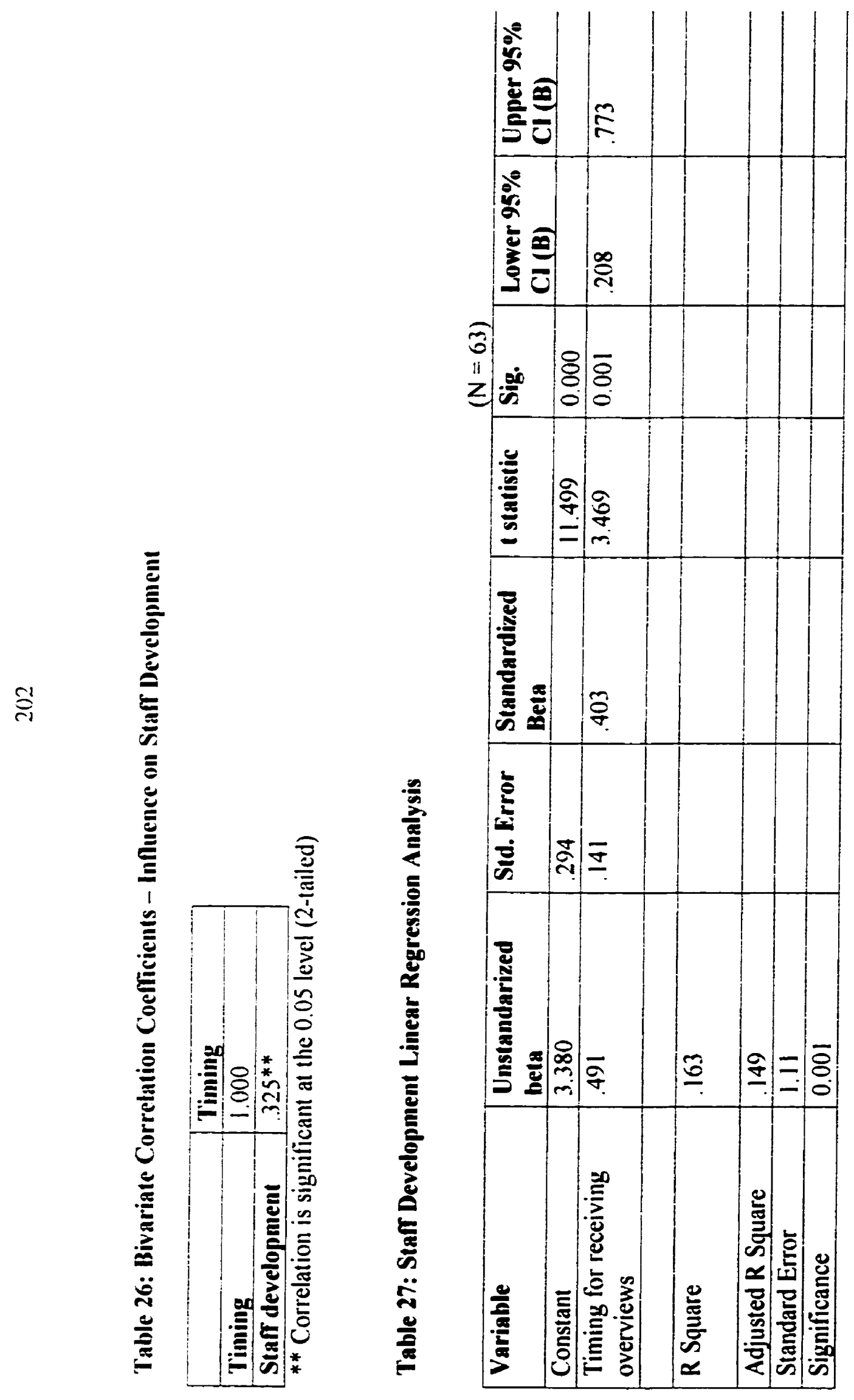




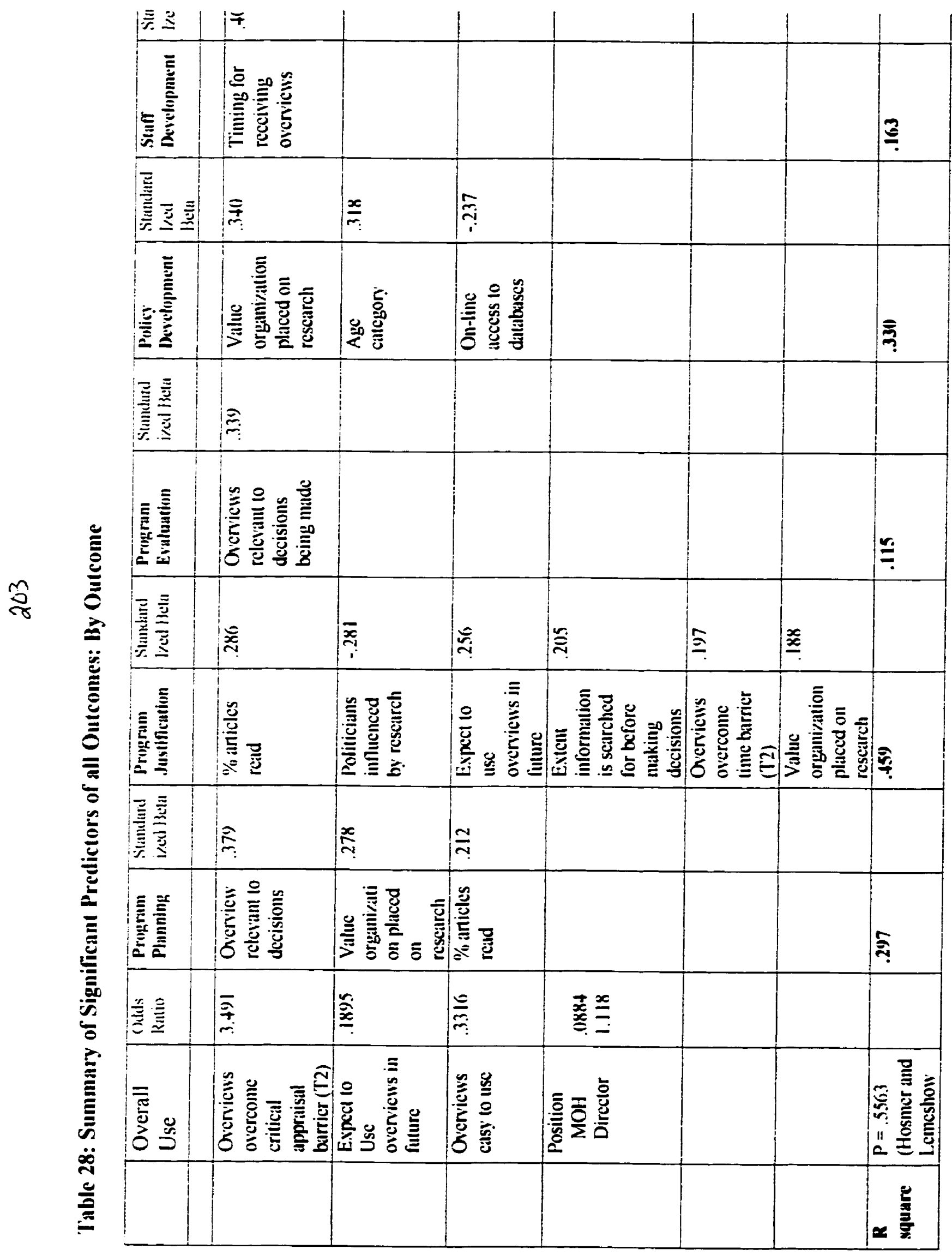




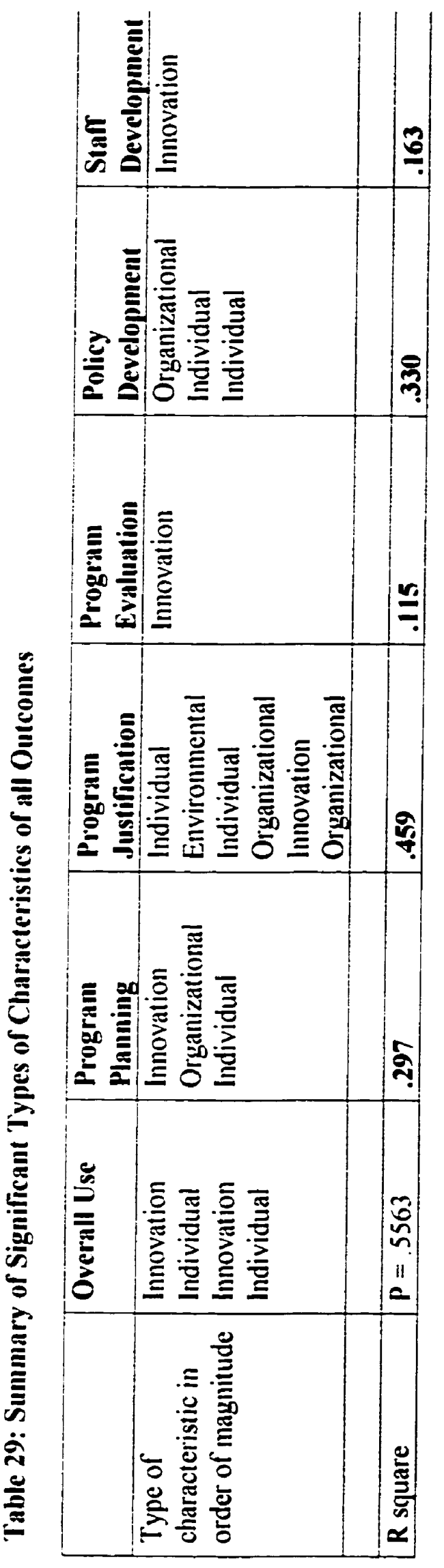




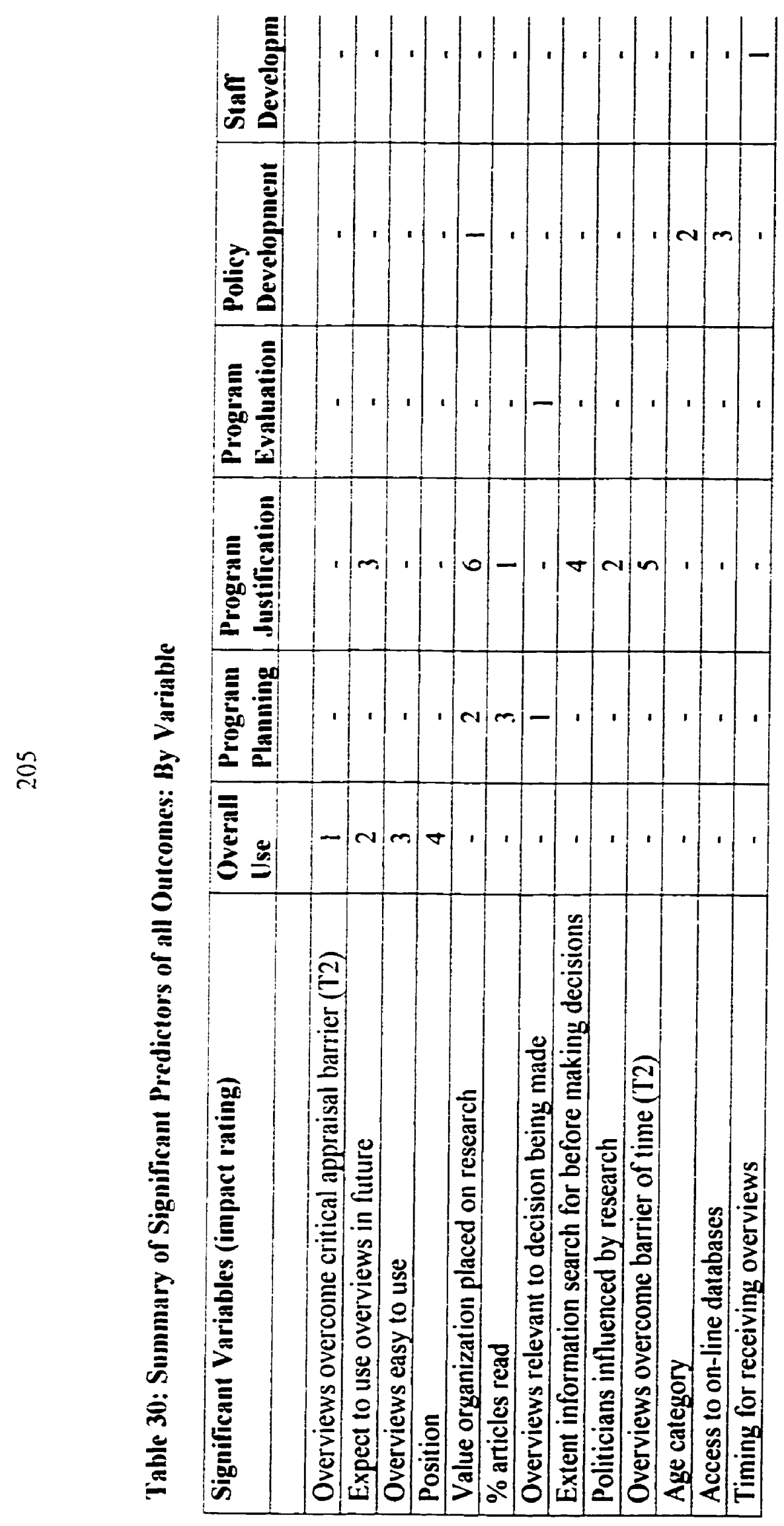




\subsection{Appendices}




\section{Appendix 1: 1998 Research Utilization Questionnaire}

\section{Research Utilization Questionnaire}

Thank you for agreeing to participate in the this study. This survey will take approximately $10-$ I 5 minutes. I will be asking you questions related to your organization, and the environment that your organization functions in. I am also interested in learning about how you have used the systematic overview(s) that was(were) sent to you approximately eighteen months ago.

I would first like to clarify the demographic information that I have for you.

21818

Position at the health department is

21766

Have been in this position for years

21767

Have been in public health for years

21769

Most recent graduation was

years ago

For the next five questions, please indicate the extent to which you Agree or Disagree with the following statements. The responses follow a five point scale starting with strongly disagree, moderately disagree, neutral or undecided, moderately agree and finally strongly agree.

21772. Overall, my health department adheres strictly to policies and procedures it has established.

Strongly Disagree Moderately Disugree Neutral/Undecided Moderately Agree Strongly Agree

21773. My health department highly values the use of research evidence to make program decisions.

Strongly Disagree Moderately Disagree NeutralUndecided Moderately Agree Strongly Agree

21774. My health department does not provide adequate resources (financial or personnel) to implement decisions that are based on scientific evidence.

Strongly Disagree Moderately Disagree Neutral/Undecided Moderately Agree Strongly Agree

21775. My health department provides ongoing training in research methods and critical appraisal to staff, to promote research utilization.

\section{Strongly Disagree Moderately Disagree Neutral/Undecided Moderately Agree Strongly Agree}


21776. Policies exist in my health department that facilitate the transfer of new information into the organization.

Strongly Disagree Moderately Disagree Neutral/Undecided Moderately Agree Strongly Agree

21783. To what extent does your health unit routinely look for information before making decisions.

Not at all

12

Moderately

3

4

$\mathbf{5}$

6

Extensively

7

21786 How important are the results from scientific research in making decisions at your health unit?

Not at all important

1 $2 \quad$ Moderately important 56

Very important

67

21815. In your opinion, what percentage of public health departments in Ontario do you think use systematic overviews to make program decisions?

$$
\%
$$

21816. In your opinion, what percentage of public health decision-makers in the same position as yourself do you think use systematic overviews to make program decisions. 
I am now going to ask you six questions about the environment in which your health department functions. Please indicate the extent to which you Agree or Disagree with the following statements. The response categories begin with strongly disagree, moderately disagree, neutral or undecided, moderately agree and strongly agree.

21790. Provincial and municipal regulations and legislation greatly impact on the decisions my health department makes about public health programs.

Strongly Disagree Moderately Disagree Neutral/Undecided Moderately Agree Strongly Agree

2179 I Most program decisions made at my health department are made in collaboration with other local health institutions or community agencies.

Strongly Disagree Moderately Disagree Neutral/Undecided Moderately Agree Strongly Agree

21792. The Medical Officer of Health of my health department has a great deal of influence over the decisions made by the local board of health.

Strongly Disagree Moderately Disagree Neutral/Undecided Moderately Agree Strongly Agree

21793. The Medical Officer of Health of my health department has a great deal of influence over the decisions made by the municipal/local politicians.

Strongly Disagree Moderately Disagree Neutral/Undecided Moderately Agree Strongly Agree

21794. Our local board of health is influenced by rigorous scientific evidence when making decisions about public health programs.

Strongly Disagree Moderately Disagree Neutral/Undecided Moderately Agree Strongly Agree

21795. Our local city council or politicians are influenced by rigorous scientific evidence when making decisions about public health programs.

Strongly Disagree Moderately Disagree Neutral/Undecided Moderately Agree Strongly Agree 
In the final part of this survey I am going to ask questions about how you have used the systematic overview(s) that were sent to you during the summer of 1996 . You may have received any of five of the following systematic overviews:

1) Effectiveness of Public Health Nursing Interventions in Parent-Child Health: A Systematic Overview of Literature Reviews;

2) A Systematic Overview of the Effectiveness of Public Health Nursing Interventions: An Overview of Adolescent Suicide Prevention Programs;

3) The Effectiveness of Community-Based Heart Health Projects: A Systematic Overview;

4) A Systematic Overview of the Effectiveness of Home Visiting as a Delivery Strategy for Public Health Nursing Interventions;

5) A Systematic Overview of the Effectiveness of Community-Based Projects.

If you received more than one systematic overview please chose one to answer the following questions.

21789 Do you recall receiving a systematic overview(s) from the Hamilton-Wentworth Department of Public Health Services approximately two years ago?

If answer is yes continue with question $\$ 22$.

If answer is no skip to question $\# 32$

Please use these responses to answer the following question. Nothing, Read, Considering Using. Passed on to other. Used to make a program decision

If answer if anything but nothing, continue with question \#23.

If answer is Nothing skip to question $\# 32$.

Use the following responses to answer the next five questions. Not at all, to some extent, a lot, a great deal.

21806. To what extent did the systematic overview influence decisions related to program planning?

$\begin{array}{llll}\text { Not at all } & \text { To some extent } & \text { A lot great deal }\end{array}$

21807. To what extent did the systematic overview influence decisions related to program justification?

Not at all To some extent A lot A great deal

21808. To what extent did the systematic overview influence decisions related to program evaluation?

$\begin{array}{llll}\text { Not at all } & \text { To some extent }\end{array}$ 
2 I 809. To what extent did the systematic overview influence decisions related to policy development?

$\begin{array}{llll}\text { Not at all } & \text { To some extent } & \text { A great deal }\end{array}$

21810. To what extent did the systematic overview influence decisions related to staff development?

Not at all

To some extent

A lot

A great deal

Thank you for taking the time to complete this survey. 


\section{Appendix 2: 1998 Organizational Demographics Questionnaire \\ Organizational Demographics Survey}

I am going to ask you a series of questions related to your organization. Please answer these questions as you currently perceive your organization.

11315 What is the total number of employees that work in your health department, including professional and support staff?

11317 Is the area served by your public health department predominately rural or urban?

11318 How many divisions, such as nursing, environmental, healthy lifestyles, are there in your health department?

11319 On the whole, how many programs, such as sexual health, heart health, food safety, are delivered through your health department?

11320 Starting with the front line workers and proceeding to the Medical Officer of Health, how many levels of workers are there in your health department?

11322 Is your health department currently involved in any research studies, projects or program evaluations?

11321 How many research projects, in total, are currently being conducted in your health department

None $\quad 1-4 \quad 5-10 \quad$ More than 10.

11324 Teaching Health Unit Status: Yes/No

Thank you for providing this information for this study. 
Appendix 3: Ontario Health Care Evaluation Network: Baseline Survey 1996

20793 Which age group are you in

$\begin{array}{lllll}20-29 & 30-39 & 40-49 & 50-59 & 60-69\end{array}$

20705 Do you have easy access to someone who provides help in becoming familiar with. interpreting, or utilizing research?

20707 In the past month, approximately how many journal articles have you retrieved?
None
$<10$
11- 19
20-29
$>30$

20708 In the past month, on average, what percentage of retrieved articles do you find you actually read?

None $\quad<25 \% \quad 25-50 \% \quad 51-75 \% \quad>75 \%$

20715 In the past year, how many days have you committed to attending conferences or workshops?

20722 How much of a barrier is limited critical appraisal skills for you in using research in your daily practice?

Not a problem minor barrier moderate barrier serious barrier very serious barrier

20727 How much is limited financial resources a barrier to using research in your daily practice?

Not a problem minor barrier moderate barrier serious barrier very serious barrier

20709 Do you have access to on-line database searching?

20745 Have you used systematic overviews in the past to make decisions with?

20765 What priority do you think should be given to conducting systematic overviews?
Bottom
low
middle
high
top

20734 How much weight is research given in your health unit for making decisions?
$0-20 \%$
$21-40 \%$
$41-60 \%$
$61-80 \%$
$81-100 \%$

20735 How much weight do you personally believe research should have?
$\mathbf{0 - 2 0 \%}$
$21-40 \%$
$41-60 \%$
$61-80 \%$
$81-100 \%$

20479 How likely do you think systematic overviews would overcome the barrier of limited access to research literature?
Definitely won't
probably won't
may
probably will 
2075 i How likely do you think systematic overviews would overcome the barrier of limited time to review research literature?

Definitely won't probably won't may probably will definitely won't

20752 How likely do you think systematic overviews would overcome the barrier of limited critical appraisal skills?

Definitely won't probably won't may probably will definitely won't

20757 How likely do you think systematic overviews would overcome the barrier of limited resources?

Definitely won't probably won't may probably will definitely won't

20754 How likely do you think systematic overviews would overcome the barrier of lack of research relevance to decisions being made?

Definitely won't probably won't may probably will definitely won't

20755 How likely do you think systematic overviews would overcome the barrier of unsupportive work cultures?

Definitely won't probably won't may probably will definitely won't

20756 How likely do you think systematic overviews would overcome the barrier of lack of authority to make decisions?

Definitely won't probably won't may probably will definitely won't 


\section{Appendix 4: Ontario Health Care Evaluation Network: Follow-up Survey 1996}

20465 Do you think systematic overviews would overcome the barrier of limited authority to make decisions?

Definitely won't possible won't no impact possibly will definitely will

20461 Do you think systematic overviews would overcome the barrier of limited critical appraisal skills?

Definitely won't possible won't no impact possibly will definitely will

20455 In the past three months have you used any other systematic overviews in your work?

20479 What priority do you think should be given to conducting systematic overviews?

Top high middle low bottom

20459 Do you think systematic overviews would overcome the barrier of limited access to research literature?

Definitely won't possible won't no impact possibly will definitely will

20458 Do you think systematic overviews would overcome the barrier of time?

Definitely won't possible won't no impact possibly will definitely will

20466 Do you think systematic overviews would overcome the barrier of limited financial resources?

Definitely won't possible won't no impact possibly will definitely will

20462 Do you think systematic overviews would overcome the barrier of lack of research relevance to decisions being made?

Definitely won't possible won't no impact possibly will definitely will

20464 Do you think systematic overviews would overcome the barrier of an unsupprotive work culture to using research in practice?

Definitely won't possible won't no impact possibly will

definitely will

20445 How would you rate the systematic overviews you received for ease of use?
Excellent
$\operatorname{good}$
moderate
fair
poor 
20446 How would you rate the systematic overviews you received for timing?

Excellent

good

moderate

fair

poor

20447 How would you rate the systematic overviews you received for relevance to decisions being made?

Excellent

good

moderate

fair

poor

20428 Do you expect to use the systematic overviews in the future 


\section{University of Toronto}

OFFICE OF RESEARCH SERVICES

\section{PROTOCOL REFERENCE $\$ 3679$}

June 2. 1998

Professor R. Cockerill

Health Administration

MeMurrich Building, 2nd Floor

12 Queen's Park Crescent West

University of Toronto

Dear Professor Cockerill:

Re: $\quad$ "Facilitating Evidence-Based Decision-Making in Public Health Administration Departments in Ontario" by M. Dobbins, R. Cockerill (supervisor)

We are writing to advise you that a Review Committee composed of Drs. L. O'Brien-Pallas. L. Yuan and Professor D. Craig has granted approval to the above-named research study.

The approved consent forms are attached. Subjects should receive a copy of their consent form.

During the course of the research. any significant deviations from the approved protocol (that is, any deviation which would lead to an increase in risk or a decrease in benefit to human subjects) and/or any unanticipated developments within the research should be brought to the attention of the Office of Research Services.

Best wishes for the successful completion of your project.

Yours sincerely.

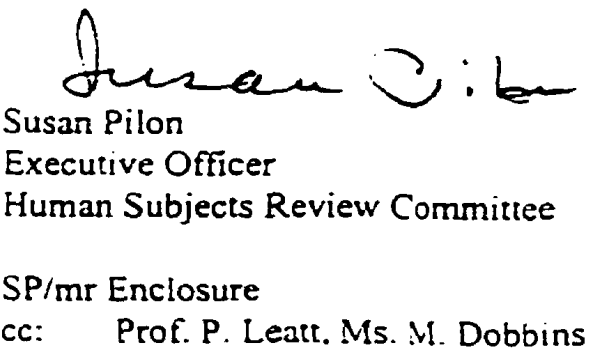


Dear :

\title{
Re: Facilitating Evidence-Based Decision-Making in Public Health Departments in Ontario
}

\author{
A study funded, in part, by the Central East Health Intelligence Unit
}

\section{Background}

The first phase of this study: TRANSFERRING PUBLIC HEALTH RESEARCH TO HEALTH SYSTEMS PLANINING - ASSESSING THE RELAVNCE AND ACCESSIBILITY OF

SYSTEMATIC OVERVIEWS, was conducted in 1996/97 by the Hamilton-Wentworth Department of Public Health Services. This descriptive study was funded by the Ontario Health Care Evaluation Network. The purpose of this study was to describe what decision-makers thought about systematic overviews as a dissemination strategy and whether they felt systematic overviews facilitated research use in program decision-making.

Phase two of this research is about to begin. The purpose of this second study is to describe how the systematic overviews disseminated in the previous study have bee used over the last eighteen months, as well as to examine the relationships between individual, organizational, environmental, and innovation characteristics, and research utilization in public health departments in Ontario. Another important objective of this study is to develop a comprehensive definition of the term evidence-based decision-making and determine how it impacts on research utilization.

I am contacting you to give you information about this study and to request your assistance in pre-testing the study questionnaires and tools. This pre-test is for the purposes of ensuring valid and reliable research tools, and your response will not be included in the study results.

\section{Participants}

Participation in the pre-testing phase is being requested from selected members of four groups of decision-makers, who make decision related to public health nursing practice: the Medical Officer of Health, Associate Medical Officers of Health, Program Directors, and Program Managers. These four groups match those who will receive the final questionnaires.

\section{What is involved?}

\section{Research Utilization Questionnaire}

Your participation would involve two telephone calls of 15-20 minutes answering questions about organizational and environmental characteristics of your organization, as well as how you have used the systematic overviews in your daily practice. The information you provide will be kept completely confidential and will not be used in any analysis of study results. It will only be used to measure the reliability of the questionnaire and to further refine it. 


\section{Confidentiality}

Your name will be recorded on a sheet matching names with code numbers. Only the code number will be marked on the record of your response given by phone. The list matching names with code numbers will be kept only by the research assistant and will be destroyed when the pre-test phase in completed. Your answers will not be reported in any reports of study results.

\section{Follow up}

I will be contacting you by phone in approximately one week, to ask for your consent and to set a time for the initial phone appointment should you be willing to participate.

If you have any further questions, please call me at $905546-3531$.

Thank you for your time and cooperation. We hope that this study will facilitate research utilization and evidence-based decision-making and ultimately improve the effectiveness and efficiency of public health programs across Ontario and Canada.

Yours Sincerely,

Maureen Dobbins, RN, BScN

PhD Student, University of Toronto

Department of Health Administration 
Appendix 7: Introduction and Consent form for Public Health Department Participation

Consent to be obtained from the Medical Officer of Health

\author{
Re: Facilitating Evidence-Based Decision-Making \\ in Public Health Departments in Ontario
}

\author{
A study funded, in part, by the Central East Health Intelligence Unit
}

Dear

I am writing to request your public health department's participation in a study looking at ways to facilitate evidence-based decision-making and research utilization in Public Health in Ontario.

During the summer of 1996, your public health department participated in a study entitled: TRANSFERRING PUBLIC HEALTH RESEARCH TO HEALTH SYSTEMS PLANNING ASSESSING THE RELAVNCE AND ACCESSIBILITY OF SYSTEMATIC OVERVIEWS, conducted by the Hamilton-Wentworth Department of Public Health Services. This descriptive study was funded by the Ontario Health Care Evaluation Network. The purpose of this study was to describe what decision-makers thought about systematic overviews as a dissemination strategy and whether they felt systematic overviews facilitated research use in program decision-making.

Phase 2 of the previous study is about to begin. The purpose of this second study is to describe how the systematic overviews disseminated in the previous study have bee used over the last eighteen months, as well as to examine the relationships between individual, organizational, environmental, and innovation characteristics and research utilization in public health departments in Ontario. Another important objective of this study is to gain a more comprehensive understanding of the term evidence-based decision-making and how it impacts on research utilization.

This study has been funded, in part, by the Central East Health Information Planning Project, and will provide the data for the primary investigator's doctoral dissertation. It has passed ethics review by the University of Toronto Committee for Ethics in Research. It is designed as a descriptive survey of Ontario Public Health decision-makers who make decisions related to public health nursing practice. The study will include a 20 minute telephone survey to all decision-makers in your health department who make decisions related to public health nursing practice, who participated in the Hayward, et al study mentioned above, and a short, selfadministered mailed questionnaire regarding organizational demographic data, to be completed by your administrative assistant

The previous study concentrated on collecting data related to individual characteristics. However, the research utilization literature suggests that organizational and environmental characteristics are significantly associated with research use. This type of data has not previously been collected in the public health field. The primary unit of analysis will be the public health department, however, it will be impossible to identify health departments or individuals within 
health departments in the study report. The data will give important information about the experience, opinions and needs of 225 people in leadership positions and will facilitate the development of effective research dissemination strategies to meet those needs.

The questionnaires have been developed from previous research utilization studies, and have been pre-tested with the Medical Officer of Health, Associate Medical Officer of Health, Program Directors and Managers at the Hamilton-Wentworth Department of Public Heaith Services. The telephone survey and face-to-face interviews will be conducted by myself.

Dr. Marilyn James, Dr. Larry Chambers, and Dr. John McGurran have supported the development and implementation of this project and are also able to respond to queries about the study purpose and methods.

If you have any questions or concerns, please do not hesitate to contact me at $905546-3531$.

I hope this information is helpful and will facilitate the participation of your staff and yourself. I and my colleagues at the Hamilton-Wentworth Public Health Department believe that the information gathered through this study can lead to more efficient use of time and resources for all of us, and better Public Health programming in the long run.

Your Sincerely,

Maureen Dobbins, RN, BScN

PhD Student. University of Toronto

Deparment of Health Administration 


\title{
Appendix 8: Introduction Letter and Consent Form for Public Health Decision-Makers \\ Re: Facilitating Evidence-Based Decision-Making in Public Health Departments in Ontario
}

\author{
A study funded, in part, by the Central East Health Intelligence Unit
}

Dear

I am writing to request your participation in a study to find out how best to facilitate evidencebased decision-making and research utilization by decision-makers in Public Health in Ontario. This study has been funded, in part, by the Central East Health Intelligence Unit, and will provide the data for the primary investigator's doctoral dissertation. It has passed ethics review by the Lniversity of Toronto Committee for Ethics in Research.

\section{Background}

The first phase of this study: TRANSFERRING PUBLIC HEALTH RESEARCH TO HEALTH SYSTEMS PLANNING - ASSESSING THE RELAVNCE AND ACCESSIBILITY OF SYSTEMATIC OVERVIEWS, was conducted in 1996/97 by the Hamilton-Wentworth Department of Public Health Services by Sarah Hayward and myself. This descriptive study was funded by the Ontario Health Care Evaluation Network. The purpose of this study was to describe what decision-makers thought about systematic overviews as a dissemination strategy and whether they felt systematic overviews facilitated research use in program decision-making.

The purpose of this second study is to describe how the systematic overviews disseminated in the previous study have bee used over the past eighteen months, as well as to examine the relationships between individual, organizational, environmental, and innovation characteristics and research utilization in public health departments in Ontario. Another important objective of this study is to gain a more comprehensive understanding of the term evidence-based decisionmaking and how it impacts on research utilization.

\section{Participants}

Participation is being requested from all members of four groups of decision-makers across Ontario: Medical Officer of Health. Associate Medical Officers of Health, Program Directors, and Program Managers, who participated in the Hayward et al study discussed above.

\section{What is involved? \\ Research Utilization Questionnaire}

You may be asked to participate in up to three different research activities. The majority of participants will only be asked to participate in one telephone survey of 15-20 minutes answering questions about organizational and environmental characteristics, as well as how they have used the systematic overviews in daily practice. The information you provide will be kept completely confidential.

\section{Confidentiality}


Your name will be recorded on a sheet matching names with code numbers. Only the code number will be marked on the record of your responses. The list matching names with code numbers will be kept only by the research assistant and will be destroyed at the end of the study.

The primary unit of analysis will be the public health department, however, it will be impossible to identify health departments or individuals within health departments in the study report. The data will give important information about the experiences, opinions and needs of 225 people in leadership positions and will facilitate the development of effective research dissemination strategies to meet those needs.

The questionnaires have been developed from previous research utilization studies, and have been pre-tested with the Medical Officer of Health. Associate Medical Officer of Health. Program Directors and Managers at the Hamilton-Wentworth Department of Public Health Services. The telephone survey and face-to-face interviews will be conducted by myself.

Dr. Marilyn James, Dr. Larry Chambers, and Dr. John McGurran have supported the development and implementation of this project and are also able to respond to queries about the study purpose and methods.

If you have any questions or concerns, please do not hesitate to contact me at 905 546-3531.

I hope this information is helpful and will facilitate your participation. I believe that the information gathered through this study can lead to more efficient use of time and resources for all of us, and better Public Health programming in the long run.

Your Sincerely,

Maureen Dobbins, RN, BScN

PhD Student, University of Toronto

Department of Health Administration 